\title{
A CRISPR Screen Identifies Myc-associated Zinc Finger Protein (MAZ) as an Insulator Functioning at CTCF boundaries in Hox Clusters
}

Havva Ortabozkoyun-Kara ${ }^{1,2}$, Pin-Yao Huang ${ }^{1,2}$, Hyunwoo $\mathrm{Cho}^{3,4,5}$, Varun Narendra ${ }^{6}$, Gary Leroy $^{1,2}$, Jane A. Skok ${ }^{3}$, Aristotelis Tsirigos ${ }^{3,4,7}$, Esteban O. Mazzoni ${ }^{8}$ and Danny Reinberg ${ }^{1,2, *}$

${ }^{1}$ Howard Hughes Medical Institute, New York University School of Medicine, New York, New York 10016, USA

${ }^{2}$ Department of Biochemistry and Molecular Pharmacology, New York University School of Medicine, New York, New York 10016, USA

${ }^{3}$ Department of Pathology, New York University School of Medicine, New York, NY 10016, USA

${ }^{4}$ Applied Bioinformatics Laboratories, New York University School of Medicine, New York, NY, USA

${ }^{5}$ Department of Radiation Oncology, New York University School of Medicine, New York, NY, USA

${ }^{6}$ Department of Medicine, Memorial Sloan Kettering Cancer Center, New York, NY, USA

${ }^{7}$ Institute for Computational Medicine, NYU School of Medicine, New York, NY 10016

${ }^{8}$ Department of Biology, New York University, New York, NY 10003, USA

*To whom correspondence should be addressed: Danny.Reinberg@nyulangone.org 


\section{Abstract}

The essential CCCTC-binding factor (CTCF) is critical to three-dimensional (3D) genome organization. CTCF binding insulates active and repressed genes within the Hox clusters upon differentiation, but such binding does not participate in boundary formation in all cell types, such

as embryonic stem cells. We conducted a genome-wide CRISPR knockout screen to identify genes required for CTCF boundary activity at the HoxA cluster, complemented by novel biochemical approaches. This screen identified Myc-associated zinc finger protein (MAZ) as a CTCF insulator co-factor, among other candidates listed herein. MAZ depletion or specific deletion of MAZ motifs within the Hox clusters led to de-repression of posterior Hox genes immediately after CTCF boundaries upon differentiation, which phenocopied deletion of the proximal CTCF motifs. Similar to CTCF, MAZ interacted with the cohesin subunit, RAD21. Upon loss of MAZ, local contacts within topologically associated domains (TADs) were disrupted, as evidenced by HiC analysis. Thus, MAZ is a novel factor sharing insulation properties with CTCF and contributing to the genomic architectural organization.

\section{One Sentence Summary}

MAZ is identified as an insulator functioning at CTCF boundaries delimiting active and repressed genes at Hox clusters 


\section{Main Text}

Beyond the DNA sequence, chromatin structure and spatial organization of the genome regulate transcriptional output. Genomes of higher eukaryotes must be tightly folded and packaged within the nucleus (1). The partitioning of the genome into independent chromatin domains occurs via insulators. While several insulators are present in Drosophila (2), CTCF is the main insulator protein in vertebrates (3-5). CTCF is a highly conserved, ubiquitously expressed, eleven-zinc finger protein (6) that is critical for development $(7,8)$, and enriched at the borders of topologically associating domains (TADs) (9-11). Amongst the many proteins associated with CTCF at different loci $(4,12)$, only cohesin co-localizes to most CTCF binding sites and is required for CTCF function $(13,14)$. CTCF boundary activity is context-dependent (15). CTCF functions to form a strong boundary between active and repressed chromatin domains, decorated by Trithorax and Polycomb, at Hox clusters upon differentiation of mouse embryonic stem cells (mESCs) into cervical motor neurons (MNs). In general, CTCF-mediated boundaries thwart RNA polymerase II progression from active to repressed genes, thereby partitioning these antagonistic chromatin domains. Although mESCs are devoid of such chromatin barriers at Hox clusters, Hox gene boundaries clearly exhibit CTCF and cohesin occupancy $(16,17)$. Thus, during differentiation, additional regulatory factors appear necessary to foster CTCF-mediated insulation properties. We devised an unbiased genome-wide loss-of-function genetic screen involving a functional CTCF boundary within the HoxA cluster in cervical MNs.

We constructed a dual reporter ESC line (Hoxa5:a7 mESCs) containing fluorescent reporters of Hox gene expression on each side of the CTCF demarcated boundary in the HoxA cluster by using CRISPR technology (18) (Fig. 1A, and Fig. S1A). The relative expression of Hox genes can be assayed in single cells, and any activity mediating CTCF-boundary formation can be assessed in 
this unique Hoxa5:a7 dual-reporter system. As expected based on previous studies $(16,19,20)$, Hoxa5-P2A-mCherry reporter expression was induced during cervical MN differentiation, while Hoxa7-P2A-eGFP remained repressed (Fig. S1B-D). To confirm that Hoxa7-P2A-eGFP reported defects in CTCF-dependent boundary formation, we deleted CTCF binding sites between Hoxa5 and Hoxa 7 genes in ESCs (CTCF $(\Delta 5 \mid 6)$ or CTCF $(\Delta 5|6: 6| 7))$ and demonstrated the de-repression of Hoxa7-P2A-eGFP by FACS analysis and RT-qPCR, as previously reported (Fig. S1B-D) (16). The $\sim 10-15 \%$ Hoxa7-P2A-eGFP positive cells (Fig. S1), allowed for enough dynamic range to identify mutants that decrease or increase CTCF insulating properties.

To identify factors required for the integrity of the CTCF-mediated boundary, we performed an unbiased loss-of-function genetic screen on Hoxa5:a7 dual reporter ESCs using a pooled genomewide library of single-guide RNAs (sgRNAs) (21), as demonstrated in Fig. 1A. A Hoxa5:a7 ESC clone expressing $\operatorname{Cas} 9$ (Fig. S2A) was transduced with the pooled lentiviral sgRNAs at a low multiplicity of infection (MOI) of $\sim 0.4$ as applied before $(21,22)$, such that each transduced cell expressed a single sgRNA. The reporter ESCs $(\mathrm{t}=0)$ were then differentiated into cervical MNs $(\mathrm{t}=6)$ via the addition of RA/SAG (23), and sorted by FACS into two MN populations: (1) WT MNs ( $m$ Cherry positive/eGFP negative cells), $\mathrm{t}=6 \mathrm{a}$ and (2) CTCF boundary disrupted MNs (double positive cells), $\mathrm{t}=6 \mathrm{~b}$. By preparing libraries at each time-point, the relative sgRNA representation at $\mathrm{t}=0,6,6 \mathrm{a}$, and $6 \mathrm{~b}$ were compared using next generation sequencing, as described previously $(21,22,24,25)$. This screen setup enabled identification of three sets of genes: (1) Essential genes in mESCs (negative selection), (2) Essential/differentiation related genes in MNs (negative selection), and (3) Genes affecting CTCF-boundary function (positive selection). Throughout the screen, Hox gene reporter expression was not observed in ESCs upon perturbation 
of any gene, which increased our confidence in identifying CTCF-boundary related candidates rather than general repressors.

As expected from a functional screen, we observed selective loss of essential genes in the starting population (ESCs, $\mathrm{t}=0$ ) compared to plasmid library (Fig. 1B, and Fig. S2B-C), and further loss of genes essential/required for $\mathrm{MN}$ differentiation $(\mathrm{MN}, \mathrm{t}=6)$ compared to $\mathrm{ESCs}(\mathrm{t}=0)$ population (Fig. 1C, and Fig. S2D-E), indicating the success of the screen. Among genes underrepresented in MNs compared to ESCs (FDR<0.05), we observed Polycomb group genes, CTCF, cohesin components, and several components related to the $\mathrm{MN}$ differentiation pathway. Our genome-wide screens were performed in duplicates by using independent genome-wide sub-libraries (library A, and library B), resulting in four independent screens. In each screen, we identified $\sim 1000$ genes positively selected in CTCF-boundary disrupted MNs $(t=6 \mathrm{~b})$ compared to WT MNs ( $\mathrm{t}=6 \mathrm{a})$ by using the Model Based Analysis of Genome-wide CRISPR Knock-out Screen (MaGeCK) tools $(26,27)$. We narrowed the candidate list to 215 genes in CTCF-boundary disrupted MNs compared to WT MNs out of four independent sub-library screens, Fig. 1D. Notably, Maz was identified as a top candidate $(\mathrm{rank}=2)$ in one of the genome-wide screens, and also detected in similar screen $($ rank=486) (Fig. 1D and Data S1).

We complemented the genetic screen with orthogonal biochemical approaches for the identification of proteins co-localizing with CTCF on chromatin. Unlike previous studies to identify CTCF partner proteins in soluble cellular fractions through the use of overexpression based systems $(12,28)$, we identified proteins co-localizing with CTCF on chromatin that may or may not interact with CTCF, but nonetheless may be important for its insulation properties in situ. To pull-down CTCF under endogenous conditions, we generated an ESC line containing Cterminal FLAG-tagged CTCF via CRISPR technology (Fig. S3A) (18), and confirmed successful 
FLAG-CTCF immunoprecipitation (IP) from the nuclear fraction of ESCs (Fig. S3B, and see Fig. S3D). To expand and identify factors co-localizing with CTCF on chromatin, we applied two biochemical methods: (1) FLAG-CTCF immunoprecipitation (IP) from native chromatin in ESCs and MNs (Fig. S3C), (2) FLAG-CTCF immunoprecipitation (IP) from crosslinked chromatin in ESCs and MNs (Fig. 1E), an adapted version of the chromatin immunoprecipitation (ChIP)-mass spectrometry (ChIP-MS) approach described previously (29-32). Affinity purification performed here enriched for CTCF-bound chromatin fragments using FLAG pull-down followed by FLAG peptide elution to minimize nonspecific interactions. We then performed mass spectrometry (MS) on the eluted proteins. In both ESCs and MNs, wild-type cells without FLAG-tag acted as background controls to normalize FLAG IPs. In both Flag-CTCF ChIP-MS approaches, we identified known interactors and novel proteins interacting or co-binding with CTCF (Fig. 1E-F, Fig. S3C, and see Data S2 for all candidates). As expected, we recovered cohesin components and accessory subunits, and other chromatin remodelers (Fig. 1F and Fig. S3C). Interestingly, MAZ was identified uniquely in the crosslinked based CTCF ChIP-MS and served as an overlapping candidate based on the Hox related functional screen and CTCF ChIP-MS approach. Thus, Maz might serve a role in regulating the CTCF-boundary at the Hox loci. MAZ was also reported as co-localizing with CTCF at $\sim 48 \%$ of binding sites based on ENCODE ChIP sequencing (ChIPseq) data in K562 cells (33), reinforcing our observation of their proximal binding on the crosslinked chromatin.

Both genetic and biochemical approaches revealed a large list of candidates, which were further narrowed down and validated through independent secondary genetic screens. In order to systematically narrow down candidates from the primary genome-wide screens (Data S1), and check whether CTCF partners identified in Fig. 1F and Fig. S3C (Data S2) have a role at the 
CTCF-boundary at the HoxA cluster, we performed secondary loss-of-function screens with a small custom library (Data S3). This custom library included sgRNAs targeting the list of candidates from primary screens, and other proteins that co-purified with CTCF (Fig. S4E). The sgRNAs in the custom library were retrieved from another genome-wide library constructed with improved design tools (34). Importantly, these secondary screens were performed with increased statistical power in ESCs having either the wild-type Hoxa5:7 reporter (Fig. 2A, and Fig. S4A-B), or the CTCF $(\Delta 5|6: 6| 7)$ Hoxa5:7 reporter (Fig. 2D, and Fig. S4C-D) to focus on candidates uniquely impacting the CTCF-boundary in the wild-type background. Based on the rank of genes overrepresented in the Hoxa5:7 dual positive MN population compared to Hoxa5 positive cells, we identified 55 genes that disrupt the CTCF-boundary in the wild-type background having intact CTCF binding sites (Fig. 2B, Data S4). Similarly, we identified 165 genes that influence the CTCF-boundary/Hoxa7 gene expression from screens performed in the CTCF $(\Delta 5|6: 6| 7)$ background (Fig. 2E, Data S5). Thus, the secondary screens resulted in a small gene list (43 genes) that when mutated phenocopied CTCF $(\Delta 5 \mid 6)$ motif deletion in the presence of intact CTCF binding sites (see Fig. S4F for comparison of secondary screens in both backgrounds, Data S6). Importantly, the secondary screens also confirmed the identification of Maz uniquely in the wildtype background. Other genes shown in Fig. 2B and Fig. 2E are expected positive controls such as Ctcf, cohesin components/accessory subunits, and Znf143 that is reported to co-localize with CTCF at TADs (35). high in multiple primary screens, identified as a co-localizing factor with CTCF on chromatin, and further validated through secondary screens. MAZ is a ubiquitously expressed protein, initially identified as a regulatory protein associated with $M y c$ gene expression (36), and also identified as 
a regulatory factor for the insulin promoter (37). To validate the screen results, we generated MAZ knock-out (KO) in mESCs through CRISPR (Fig. S5A-B). MAZ KO did not produce a profound change in gene markers associated with ESC and MN fate (Fig. S6D). In addition, MAZ KO did not result in cell cycle changes in ESCs (Fig. S6E-F). MAZ KO did not affect overall CTCF and cohesin levels (Fig. S5B). As shown in Fig. 2C, MAZ KO in MNs mimicked the specific deletion of the CTCF site $(\Delta 5|6: 6| 7)$ at the HoxA cluster and disrupted the boundary between active and repressed genes. In addition, MAZ KO resulted in differential expression of $\sim 2400$ genes in ESCs (Fig. 2G, left; see Fig. S5C for GO analysis and Data S7), and 1800 genes in MNs compared to WT (Fig. 2G, right and Data S8). Gene Ontology (GO) analysis indicated that developmental processes, particularly anterior-posterior pattern specification, are enriched in MAZ KO MNs compared to WT MNs (Fig. S5D). Consistent with a boundary role and with CTCF binding site deletions, MAZ KO led to de-repression of posterior Hox genes in MNs, but not in ESCs (Fig. 2G, see RT-qPCR data in Fig. S6A-C). We did not observe further de-repression of Hoxa7-GFP upon differentiation of CTCF ( $\Delta 5|6: 6| 7)$ mESCs having a Maz deletion into MNs (Fig. 2F, see Fig. S6G for quantification), confirming our secondary screen results (Fig. 2D-E).

Based on our ChIP-seq analysis, $\sim 20 \%$ of MAZ binding sites co-localize with CTCF in mESCs and MNs (Fig. 2H-J). MAZ mostly binds to promoters in addition to introns, intergenic regions, and other regions (Fig. S7A). As we initially identified the MAZ KO as influencing the CTCFboundary at the HoxA cluster (Fig. 2C), we compared ChIP-seq tracks of MAZ at the HoxA cluster and to those of CTCF. We observed co-localization of MAZ and CTCF at CTCF borders in Hox clusters as shown for the HoxA cluster in Fig. 3A (Fig. S9A-B for $H o x D$, and Fig. S10A-B for HoxC). As shown in Fig. 3A, MAZ KO in ESCs and MNs resulted in a slight decrease of CTCF binding at the boundary at HoxA cluster. We also observed a similar global decrease in CTCF 
binding in MAZ KO (Fig. S7B-C). Although MAZ and CTCF co-localize on the cross-linked chromatin (Fig. 1F, and Fig. 2H), they did not co-immunoprecipitate (co-IP) under native conditions (Fig. S3D-F). Interestingly, we observed that similar to CTCF, MAZ co-IPs with RAD21, the cohesin component (Fig. 4D, and Fig. S3E-F). Therefore, MAZ appears to interact with the cohesin component, and bind to DNA in the CTCF proximity.

MAZ binds to a GC-rich motif on DNA (GGGAGGG) through its zinc fingers (Fig. S7D-E) (38). We found MAZ binding motifs close to CTCF at Hox boundaries and tested whether deletion of MAZ binding motifs at a specific Hox cluster mimics deletion of a CTCF binding site in the respective Hox cluster. We reasoned that if MAZ binding close to a CTCF boundary has a direct effect on anterior-posterior patterning, deletion of its binding motif could alter Hox gene expression similar to MAZ KO in MNs. Interestingly, MAZ binding site deletions generated at Hoxa $5 \mid 6$ (Fig. 3B), Hoxd4 $\mid 8$ (Fig. S9), and Hoxc5|6 (Fig. S10) phenocopied CTCF binding site deletions at the respective boundaries without altering CTCF binding at the boundary (Fig. 3C-E, Fig. S8C, and Data S9-10 for HoxA; Fig. S9A and Fig. S9E for HoxD; and Fig. S10A and Fig. S10E for HoxC clusters). As expected, MAZ binding site deletions at Hox clusters did not influence the cell cycle in ESCs (Fig. S8A-B for HoxA, Fig. S9C-D for HoxD, and Fig. S10C-D for HoxC clusters). These results pointed to a specific role of MAZ binding in regulating Hox gene expression at CTCF boundaries in multiple Hox clusters during differentiation. When we analyzed how chromatin domains were influenced upon deletion of the MAZ binding site at the Hoxa5|6 boundary, we observed spreading of active chromatin ( $\mathrm{H} 3 \mathrm{~K} 4 \mathrm{me} 3)$ into the repressed region (H3K27me3) at the boundary, similar to the CTCF binding site deletion shown in Fig. 3E, and to that previously reported (16) (see Fig. S9A and Fig. S10A for HoxD and HoxC clusters). Similar 
to MAZ $(\Delta 5 \mid 6)$ not altering the neighboring CTCF binding or RAD21 binding (data not shown), CTCF $(\Delta 5|6: 6| 7)$ did not affect adjacent MAZ binding at the HoxA cluster (Fig. S7F).

Besides its boundary role at Hox clusters, CTCF plays a pleiotropic role in 3D genome structure. MAZ co-localizes with CTCF at $20 \%$ of MAZ binding sites in mESCs and MNs, and MAZ KO reduces CTCF binding. Thus, we examined the effect of MAZ KO in global genomic organization by performing $\mathrm{HiC}$ in WT versus MAZ KO ESCs and MNs in biological duplicates (Fig. 4, and Fig. S11 to S14). MAZ depletion resulted in alteration of local contacts within topologically associated domains (TADs) in ESCs (Fig. S11C) and MNs (Fig. 4A), indicating the contribution of MAZ to the integrity of TADs. As expected, the active (A) and inactive (B) compartments were not affected upon MAZ KO in ESCs and MNs (Fig. S11A). There were also changes in boundary scores upon MAZ KO in ESCs and MNs as shown by PCA (Fig. S11B). In addition, analysis of differential loop activity showed alterations upon MAZ KO in both cell types (Fig. 4B, and Fig. S11D), indicating the global loss of loops in MAZ KO compared to WT. Consistent with this observation, aggregate peak analysis (APA) showed that contact frequencies were decreased in MAZ KO ESCs (Fig. S11E), and MNs (Fig. 4C) compared to WT, and that these changes occurred in short ranges within $2 \mathrm{Mb}$. As CTCF is known to be present at loop anchors, we hypothesized that there might be loops bound by MAZ alone or MAZ and CTCF together. Therefore, we categorized the loops based on CTCF, MAZ, or MAZ and CTCF occupancy, and investigated how they were affected upon MAZ KO. Interestingly, the loops specific to CTCF, MAZ, or MAZ and CTCF showed a decrease in contact frequencies upon MAZ KO (Fig. 4C, and Fig. S11E). Moreover, we observed that some loop domains in larger ranges are also slightly downregulated upon MAZ KO while we did not observe local effects of MAZ KO on Hox cluster organization with respect to the CTCF and MAZ boundary, within the resolution of HiC (Fig. S12 to S14). 
While we did not detect interaction between MAZ and CTCF (Fig. S3E-F), MAZ and cohesin components did exhibit interaction (Fig. 4D), as is the case with CTCF (Fig. S3E-F, and (12)). Moreover, as deletion of either the MAZ or CTCF binding motif results in spreading of an active domain into a repressed one (Fig. 3C-E, and (16)), we speculate that MAZ, CTCF and cohesin function together to thwart progression of transcription from the respective active domain.

These results demonstrate that an unbiased genome-wide CRISPR screen coupled with biochemical approaches enable identification of novel factors that function similar to and in conjunction with CTCF. Our results place MAZ as a novel factor that functions in partitioning Hox clusters into insulated domains, wherein Trithorax and Polycomb activities are important to maintain distinct Hox gene expression. MAZ binding site deletions at active and repressed gene borders in Hox clusters phenocopy the effect of CTCF binding site deletions at Hox clusters (16, 39). Consistent with our findings, $M a z^{-/-}$mice show perinatal lethality, and developmental defects in the kidney and urinary track (40), though other phenotypes remain to be tested. Although more than 25 out of 39 Hox genes are expressed in the kidney, the role of Hox genes other than the Hox 11 paralogous group seems to be under active investigation (41). Hence, it can be informative to study possible Hox gene misexpression patterns in the kidney or urinary track in $\mathrm{Maz}^{-/-}$mice. MAZ contributes to the integrity of TADs, and contacts within TADs and loops are impacted upon loss of MAZ although the effects are not as large-scale as the loss of essential architectural proteins such as CTCF (42) or cohesin (43). In particular, MAZ appears to be present at the loop anchors with or without CTCF, and both proteins interact with cohesin components independently (Fig. 4E). Based on our preliminary transcription assays, MAZ and CTCF binding impact the progression of transcription in the presence of cohesin. Taken together, our findings point to MAZ functioning as a novel insulator factor at Hox clusters and being critical to genome organization. 
In addition to its role in impacting loop interactions, MAZ, together with adjacent CTCF and cohesin, possibly functions by blocking RNA polymerase II progression.

\section{References and Notes}

1. T. Misteli, Beyond the sequence: cellular organization of genome function. Cell 128, 787800 (2007).

2. K. Van Bortle et al., Drosophila CTCF tandemly aligns with other insulator proteins at the borders of H3K27me3 domains. Genome Res 22, 2176-2187 (2012).

3. J. E. Phillips-Cremins, V. G. Corces, Chromatin insulators: linking genome organization to cellular function. Mol Cell 50, 461-474 (2013).

4. C. T. Ong, V. G. Corces, CTCF: an architectural protein bridging genome topology and function. Nat Rev Genet 15, 234-246 (2014).

5. V. V. Lobanenkov, A novel sequence-specific DNA binding protein which interacts with three regularly spaced direct repeats of the CCCTC-motif in the 5'-flanking sequence of the chicken c-myc gene. (1990).

15 6. R. Ohlsson, R. Renkawitz, V. Lobanenkov, CTCF is a uniquely versatile transcription regulator linked to epigenetics and disease. Trends in Genetics 17, 520-527 (2001).

7. H. Heath et al., CTCF regulates cell cycle progression of alphabeta $\mathrm{T}$ cells in the thymus. EMBO J 27, 2839-2850 (2008).

8. E. Splinter et al., CTCF mediates long-range chromatin looping and local histone modification in the beta-globin locus. Genes Dev 20, 2349-2354 (2006).

9. J. R. Dixon et al., Topological domains in mammalian genomes identified by analysis of chromatin interactions. Nature 485, 376-380 (2012). 
10. E. P. Nora et al., Spatial partitioning of the regulatory landscape of the X-inactivation centre. Nature 485, 381-385 (2012).

11. F. Ciabrelli, G. Cavalli, Chromatin-driven behavior of topologically associating domains. J Mol Biol 427, 608-625 (2015).

12. J. Zlatanova, P. Caiafa, CTCF and its protein partners: divide and rule? J Cell Sci 122, 1275-1284 (2009).

13. E. D. Rubio et al., CTCF physically links cohesin to chromatin. Proc Natl Acad Sci US A 105, 8309-8314 (2008).

14. K. S. Wendt et al., Cohesin mediates transcriptional insulation by CCCTC-binding factor. Nature 451, 796-801 (2008).

15. S. Cuddapah et al., Global analysis of the insulator binding protein CTCF in chromatin barrier regions reveals demarcation of active and repressive domains. Genome Res 19, 2432 (2009).

16. V. R. Narendra, P.P.; An, D.; Raviram, R.; Skok, J.A.; Mazzoni,E.O.; Reinberg, D., CTCF establishes discrete functional chromatin domains at the Hox clusters during differentiation. Science 347, 1017-1021 (2015).

17. A. Nitzsche et al., RAD21 cooperates with pluripotency transcription factors in the maintenance of embryonic stem cell identity. PLoS One 6, e19470 (2011).

18. F. A. Ran et al., Genome engineering using the CRISPR-Cas9 system. Nat Protoc 8, 22812308 (2013).

19. J. P. Liu, E. Laufer, T. M. Jessell, Assigning the positional identity of spinal motor neurons: rostrocaudal patterning of Hox-c expression by FGFs, Gdf11, and retinoids. Neuron 32, 997-1012 (2001). 
20. E. O. Mazzoni et al., Saltatory remodeling of Hox chromatin in response to rostrocaudal patterning signals. Nat Neurosci 16, 1191-1198 (2013).

21. N. E. Sanjana, O. Shalem, F. Zhang, Improved vectors and genome-wide libraries for CRISPR screening. Nat Methods 11, $783-784$ (2014).

22. O. Shalem et al., Genome-scale CRISPR-Cas9 knockout screening in human cells. Science 343, $84-87$ (2014).

23. H. Wichterle, I. Lieberam, J. A. Porter, T. M. Jessell, Directed differentiation of embryonic stem cells into motor neurons. Cell 110, 385-397 (2002).

24. O. Shalem, N. E. Sanjana, F. Zhang, High-throughput functional genomics using CRISPRCas9. Nat Rev Genet 16, 299-311 (2015).

25. O. Parnas et al., A Genome-wide CRISPR Screen in Primary Immune Cells to Dissect Regulatory Networks. Cell 162, 675-686 (2015).

26. W. Li et al., MAGeCK enables robust identification of essential genes from genome-scale CRISPR/Cas9 knockout screens. Genome Biol 15, 554 (2014).

27. W. Li et al., Quality control, modeling, and visualization of CRISPR screens with MAGeCK-VISPR. Genome Biol 16, 281 (2015).

28. T. M. Yusufzai, H. Tagami, Y. Nakatani, G. Felsenfeld, CTCF tethers an insulator to subnuclear sites, suggesting shared insulator mechanisms across species. Mol Cell 13, 291298 (2004).

29. M. Soldi, T. Bonaldi, The ChroP Approach Combines ChIP and Mass Spectrometry to Dissect Locus-specific Proteomic Landscapes of Chromatin. Jove-Journal of Visualized Experiments, (2014). 
30. C. I. Wang et al., Chromatin proteins captured by ChIP-mass spectrometry are linked to dosage compensation in Drosophila. Nat Struct Mol Biol 20, 202-209 (2013).

31. M. R. Rafiee, C. Girardot, G. Sigismondo, J. Krijgsveld, Expanding the Circuitry of Pluripotency by Selective Isolation of Chromatin-Associated Proteins. Mol Cell 64, 624635 (2016).

32. S. Tu, G. LeRoy, D. Reinberg, Chromatin Starts to Come Clean. Mol Cell 64, 439-441 (2016).

33. K. Van Bortle et al., Insulator function and topological domain border strength scale with architectural protein occupancy. Genome Biol 15, R82 (2014).

34. J. G. Doench et al., Optimized sgRNA design to maximize activity and minimize off-target effects of CRISPR-Cas9. Nat Biotechnol 34, 184-191 (2016).

35. S. D. Bailey et al., ZNF143 provides sequence specificity to secure chromatin interactions at gene promoters. Nat Commun 2, 6186 (2015).

36. S. A. Bossone, C. Asselin, A. J. Patel, K. B. Marcu, Maz, a Zinc Finger Protein, Binds to C-Myc and C2 Gene-Sequences Regulating Transcriptional Initiation and Termination. Proceedings of the National Academy of Sciences of the United States of America 89, 74527456 (1992).

37. G. C. Kennedy, W. J. Rutter, Pur-1, a zinc-finger protein that binds to purine-rich sequences, transactivates an insulin promoter in heterologous cells. Proc. Natl. Acad. Sci. USA 89, 11498-11502 (1992).

38. J. Song et al., Two consecutive zinc fingers in $\mathrm{Sp} 1$ and in MAZ are essential for interactions with cis-elements. J Biol Chem 276, 30429-30434 (2001). 
39. V. Narendra, M. Bulajic, J. Dekker, E. O. Mazzoni, D. Reinberg, CTCF-mediated topological boundaries during development foster appropriate gene regulation. Genes Dev 30, 2657-2662 (2016).

40. M. Haller, J. Au, M. O'Neill, D. J. Lamb, 16p11.2 transcription factor MAZ is a dosagesensitive regulator of genitourinary development. Proc Natl Acad Sci U S A 115, E1849E1858 (2018).

41. D. M. Wellik, Hox genes and kidney development. Pediatr Nephrol 26, 1559-1565 (2011).

42. E. P. Nora et al., Targeted Degradation of CTCF Decouples Local Insulation of Chromosome Domains from Genomic Compartmentalization. Cell 169, 930-944 e922 (2017).

43. S. S. P. Rao et al., Cohesin Loss Eliminates All Loop Domains. Cell 171, 305-320 e324 (2017).

44. I. F. Davidson et al., DNA loop extrusion by human cohesin. Science 366, 1338-1345 (2019).

45. Y. Li et al., The structural basis for cohesin-CTCF-anchored loops. Nature 578, 472-476 (2020).

46. J. Joung et al., Genome-scale CRISPR-Cas9 knockout and transcriptional activation screening. Nat Protoc 12, 828-863 (2017).

47. O. Oksuz et al., Capturing the Onset of PRC2-Mediated Repressive Domain Formation. Mol Cell 70, 1149-1162 e1145 (2018).

48. J. D. Dignam, R. M. Lebovitz, R. G. Roeder, Accurate transcription initiation by RNA polymerase II in a soluble extract from isolated mammalian nuce. Nucleic Acids Research 11, 1475-1489 (1983). 
49. S. Tu et al., Co-repressor CBFA2T2 regulates pluripotency and germline development. Nature 534, 387-390 (2016).

50. G. LeRoy et al., LEDGF and HDGF2 relieve the nucleosome-induced barrier to transcription in differentiated cells. Sci Adv 5, eaay3068 (2019).

51. G. LeRoy, B. Rickards, S. J. Flint, The double bromodomain proteins Brd2 and Brd3 couple histone acetylation to transcription. Mol Cell 30, 51-60 (2008).

52. H. Mi, A. Muruganujan, D. Ebert, X. Huang, P. D. Thomas, PANTHER version 14: more genomes, a new PANTHER GO-slim and improvements in enrichment analysis tools. Nucleic Acids Res 47, D419-D426 (2019).

53. B. Langmead, C. Trapnell, M. Pop, S. L. Salzberg, Ultrafast and memory-efficient alignment of short DNA sequences to the human genome. Genome Biol 10, R25 (2009).

54. S. Anders, W. Huber, Differential expression analysis for sequence count data. Genome Biol 11, R106 (2010).

55. J. T. Robinson et al., Integrative genomics viewer. Nat Biotechnol 29, 24-26 (2011).

56. F. Ramirez, F. Dundar, S. Diehl, B. A. Gruning, T. Manke, deepTools: a flexible platform for exploring deep-sequencing data. Nucleic Acids Res 42, W187-191 (2014).

57. L. J. Zhu et al., ChIPpeakAnno: a Bioconductor package to annotate ChIP-seq and ChIPchip data. BMC Bioinformatics 11, 237 (2010).

58. N. T. T. Nguyen et al., RSAT 2018: regulatory sequence analysis tools 20th anniversary. Nucleic Acids Res 46, W209-W214 (2018).

59. T. L. Bailey, C. Elkan, Fitting a mixture model by expectation maximization to discover motifs in biopolymers. Proc Int Conf Intell Syst Mol Biol 2, 28-36 (1994). 
60. C. Lazaris, S. Kelly, P. Ntziachristos, I. Aifantis, A. Tsirigos, HiC-bench: comprehensive and reproducible $\mathrm{Hi}-\mathrm{C}$ data analysis designed for parameter exploration and benchmarking. BMC Genomics 18, 22 (2017).

61. B. Langmead, S. L. Salzberg, Fast gapped-read alignment with Bowtie 2. Nat Methods 9, 357-359 (2012).

62. M. Imakaev et al., Iterative correction of Hi-C data reveals hallmarks of chromosome organization. Nat Methods 9, 999-1003 (2012).

63. S. Heinz et al., Simple combinations of lineage-determining transcription factors prime cis-regulatory elements required for macrophage and B cell identities. Mol Cell 38, 576589 (2010).

64. S. Heinz et al., Transcription Elongation Can Affect Genome 3D Structure. Cell 174, 15221536 e1522 (2018).

65. A. Kloetgen et al., Three-dimensional chromatin landscapes in T cell acute lymphoblastic leukemia. Nat Genet 52, 388-400 (2020).

66. F. Ay, T. L. Bailey, W. S. Noble, Statistical confidence estimation for Hi-C data reveals

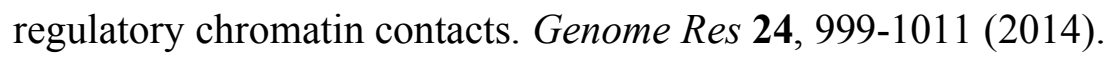

67. S. S. Rao et al., A 3D map of the human genome at kilobase resolution reveals principles of chromatin looping. Cell 159, 1665-1680 (2014).

68. M. T. Weirauch et al., Determination and inference of eukaryotic transcription factor sequence specificity. Cell 158, 1431-1443 (2014).

69. G. Ambrosini, R. Groux, P. Bucher, PWMScan: a fast tool for scanning entire genomes with a position-specific weight matrix. Bioinformatics 34, 2483-2484 (2018). 
70. F. Ramirez et al., High-resolution TADs reveal DNA sequences underlying genome organization in flies. Nat Commun 9, 189 (2018).

Acknowledgments: We thank L. Vales for reading and guidance on the manuscript. We thank S. Tu, S. Krishnan, and T. Escobar for discussions; O. Oksuz for discussions regarding sequencing data analysis; Y. Grobler for providing Drosophila S2R+ cells; and D. Hernandez for technical assistance. We thank other past and present members of the Reinberg laboratory for discussions as the work was in progress. We also thank New York University Langone Medical Center (NYULMC) Genome Technology Center, particularly A. Heguy, P. Zappile, and P. Meyn, for help with sequencing, the Applied Bioinformatics Laboratories (ABL) for providing bioinformatics support, and NYULMC Cytometry and Cell Sorting Core, particularly P. Lopez, and M. Gregory for help with FACS. This study utilized computing resources at the High-Performance Computing Facility of the Center for Health Informatics and Bioinformatics at the NYULMC. Funding: This work was supported by grants from NIH (R01NS100897) and the Howard Hughes Medical Institute to D.R; NIH(R01NS100897) to E.O.M.; American Cancer Society (RSG-15-189-01RMC), St. Baldrick's foundation (581357), and NCI/NIH (P01CA229086-01A1) to A.T; NIH (R35GM122515 and P01CA229086) to J.A.S.; NIH (F31HD090892) to H.O.K.; Alex's Lemonade Stand Foundation for Childhood Cancer to G.L.; and Memorial Sloan Kettering T32 Clinical Scholars Program to V.N. The NYULMC Genome Technology Center, the ABL, and the

NYUMC Cytometry and Cell Sorting Core are supported partially by the Cancer Center Support Grant (P30CA016087) at the Laura and Isaac Perlmutter Cancer Center. Author contributions: H.O.K., E.O.M., and D.R. conceived the project, designed the experiments, and wrote the paper; H.O.K. performed most of the experiments and the bioinformatic analysis except HiC; P.Y.H. 
helped with immunoprecipitation, transcription constructs, and RT-qPCRs; H.C. performed the bioinformatic analysis of $\mathrm{HiC}$ under the supervision of A.T.; V.N. advised in the initial design of study; G.L. advised on the mass-spectrometry and performed preliminary transcription assays; and J.A.S. advised on the progression of this study. Competing interests: D.R. was a cofounder of Constellation Pharmaceuticals and Fulcrum Therapeutics. Currently, D.R has no affiliation with either company. The authors declare that they have no other competing interests. Data and materials availability: Sequencing data has been deposited at Gene Expression Omnibus and accession numbers will be available immediately upon publication.

\section{List of Supplementary Materials}

Materials and Methods

Figs. S1 to S14

Tables S1 to S3

Captions for Data S1 to S10 
A

130,209 sgRNAs

6sgRNA/gene for 20611 genes
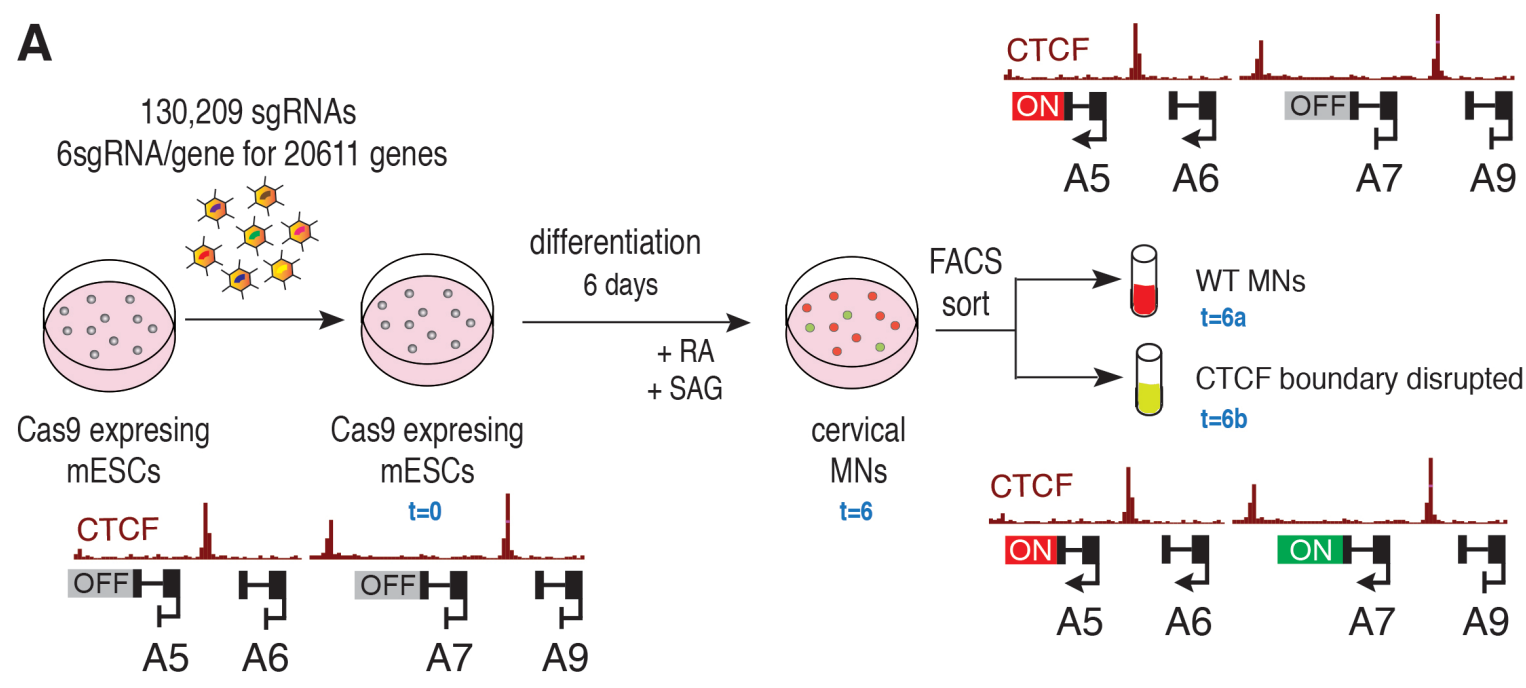

B.

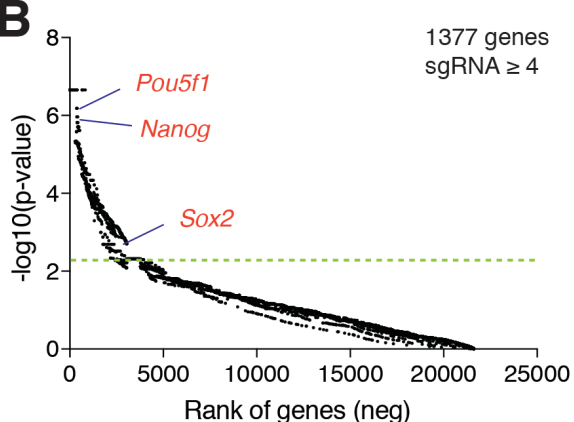

Screen
Library A
$(2 X)$

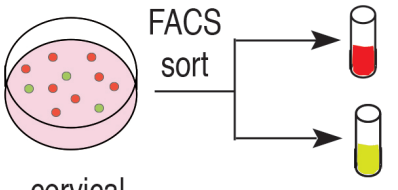

WT MNs

$\mathrm{t}=6 \mathrm{a}$

cervical

MNs

$\mathrm{t}=6$

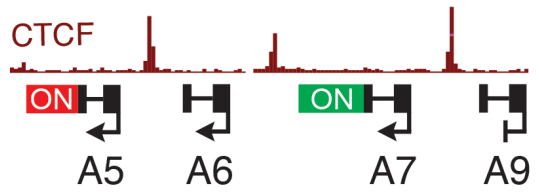

CTCF boundary disrupted MNs

$\mathrm{t}=6 \mathrm{~b}$

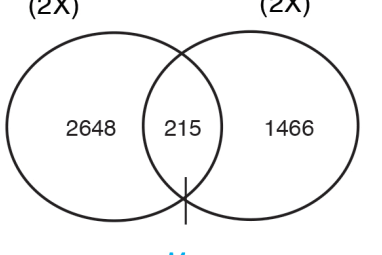

Maz

Screen $\operatorname{rank}(A)=2$

Screen $\operatorname{rank}(B)=486$

C

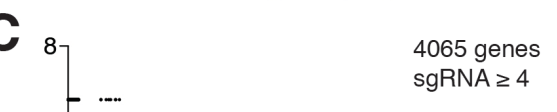

E
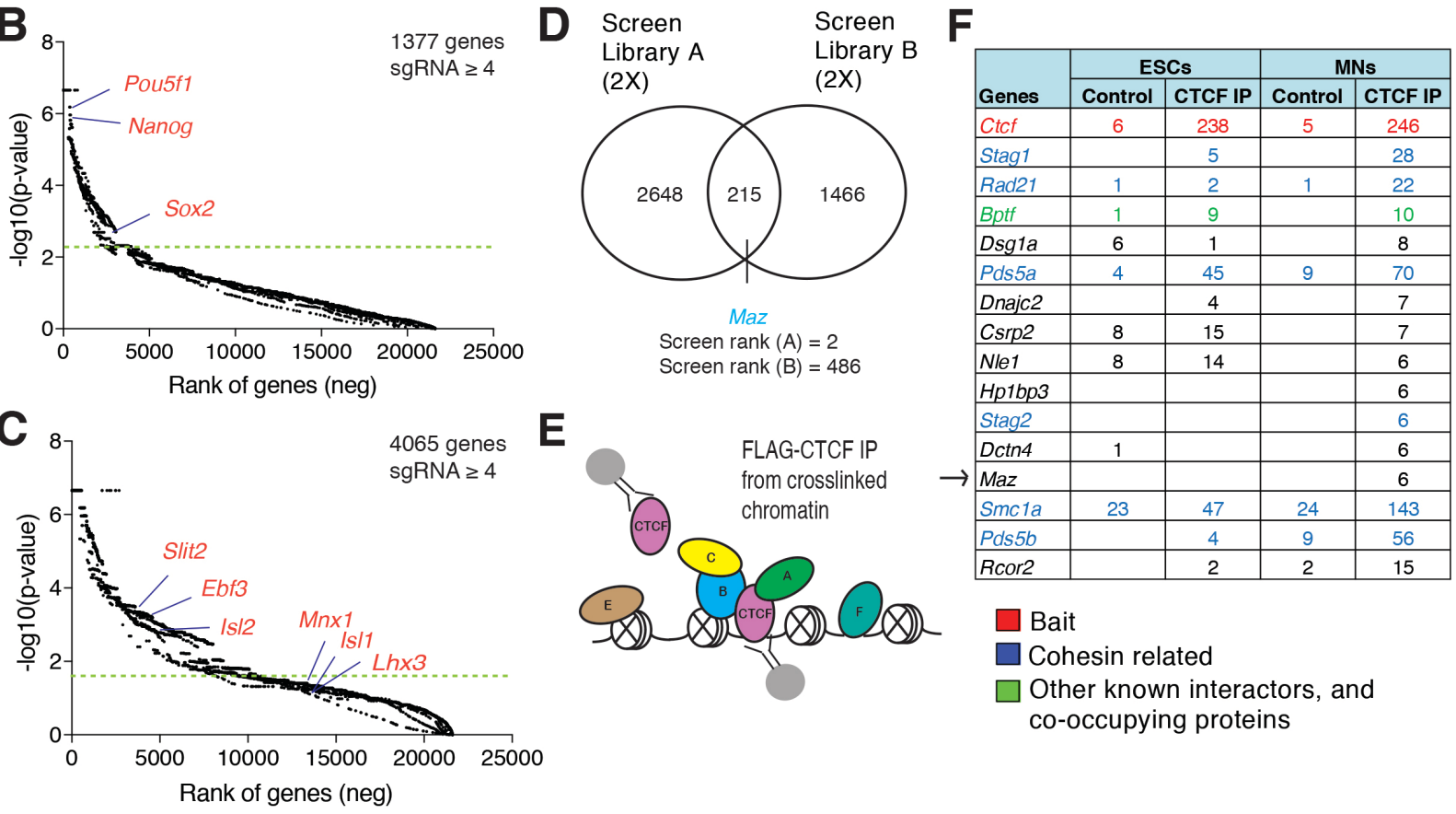

Bait

$\square$ Cohesin related

Other known interactors, and co-occupying proteins

Fig. 1. Genome-wide CRISPR loss-of-function screen to identify factors that affect the insulator function of CTCF, complemented with biochemical approaches. (A) Layout of the genetic loss-of-function screen that separates CTCF-boundary disrupted MNs from those with an intact boundary. (B) Rank of genes underrepresented in ESCs compared to plasmid library. Cutoff line indicates FDR $<0.05$. (C) Rank of genes underrepresented in MNs compared to ESCs. Cut-off line indicates FDR $<0.05$. (D) Venn diagram showing the overlap of CTCF-boundary 
related candidates identified in 4 independent (2 Library A, and 2 Library B) screens. P-value cutoff=0.05. (E) Scheme of crosslinked FLAG-CTCF ChIP-MS indicating identification of adjacent proteins on chromatin in addition to interactors. (F) Crosslinked FLAG-CTCF ChIP-MS in ESCs and MNs results in identification of known CTCF interactors, and novel proteins. The peptide counts in FLAG-CTCF IPs were normalized to control FLAG IPs in untagged cells. The list is ranked based on CTCF IP/control ratios in MNs. 
A

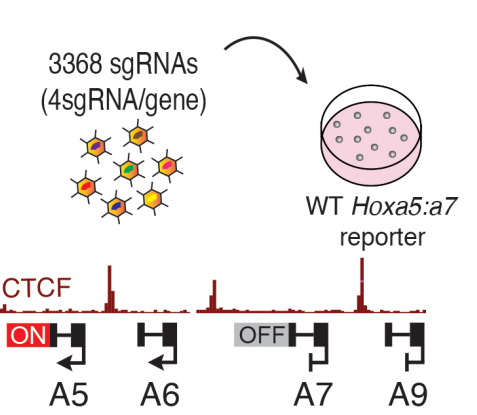

D

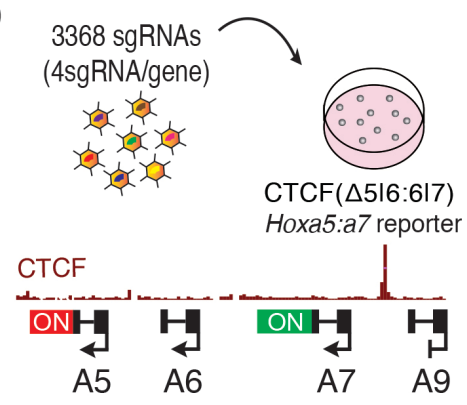

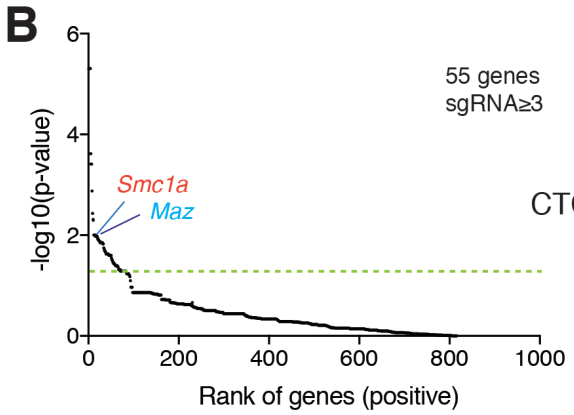

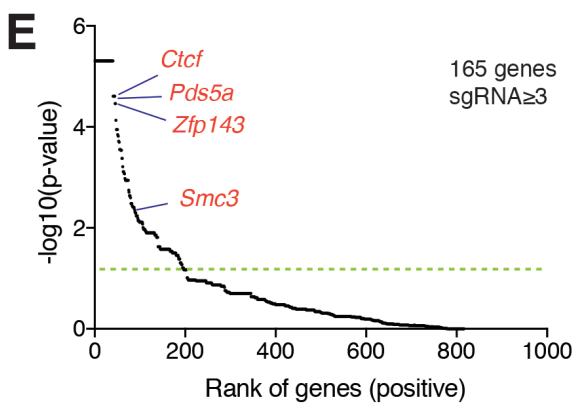

C Anterior Posterior
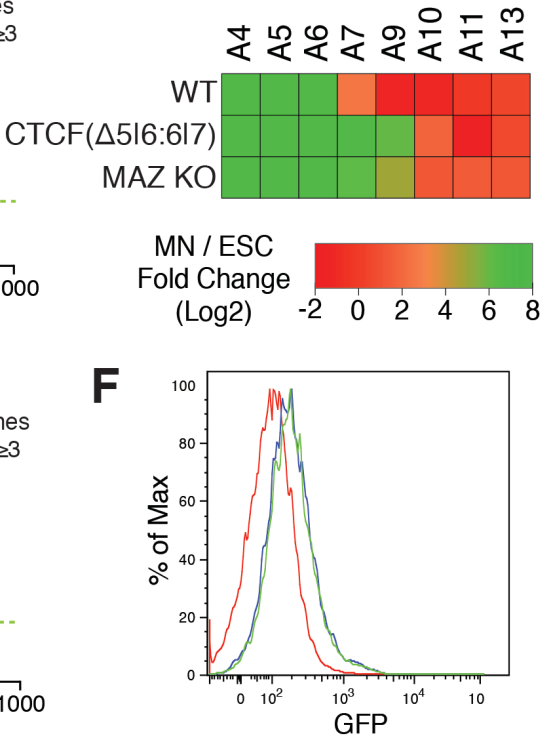

MAZ KO \& $\operatorname{CTCF}(\triangle 5 \mid 6: 617)$ $\square \operatorname{CTCF}(\triangle 516: 617)$
G

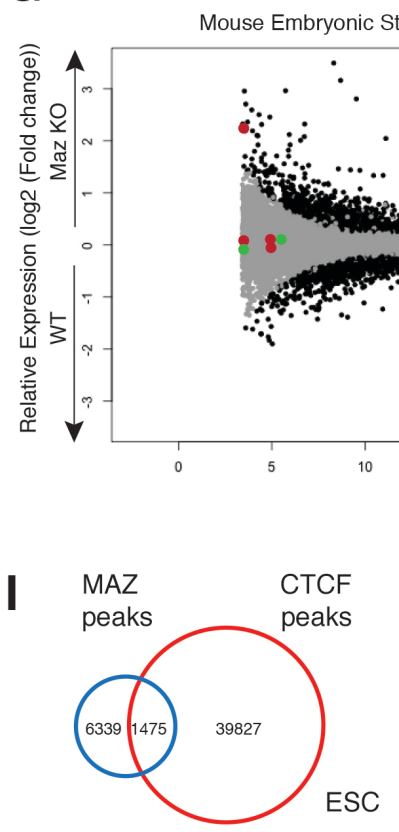

H
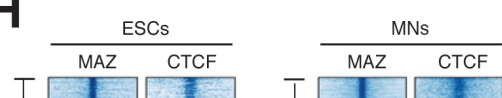
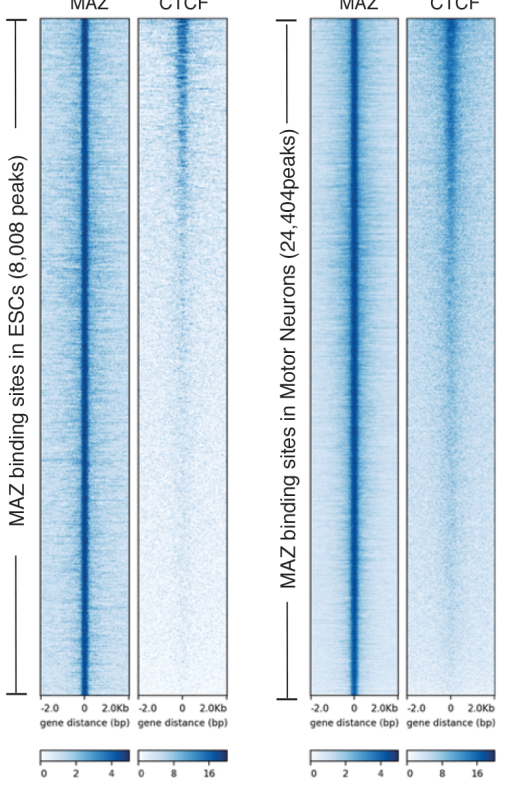

Fig. 2. Secondary CRISPR loss-of-function screens, and individual validation of MAZ as an insulator functioning at CTCF-boundaries in Hox clusters. (A) Scheme of secondary screen performed in WT background. (B) Rank of genes overrepresented in boundary disrupted MNs versus WT MNs in one biological replicate. Cut-off line indicates $\mathrm{p}<0.05$. (C) Heat map of 
relative gene expression in $\mathrm{WT}, \operatorname{CTCF}(\Delta 5|6: 6| 7)$, and $\mathrm{MAZ} \mathrm{KO}$ at the $H o x A$ cluster in MNs versus ESCs from three biological replicates. Maz KO represents three independent clones. (D) Scheme of secondary screen performed in CTCF $(\Delta 5|6: 6| 7)$ background. (E) Rank of genes overrepresented in dual positive Hoxa5:a7 MN population (further disrupted boundary) versus Hoxa5-mCherry positive population (WT) in two biological replicates. Cut-off line indicates $\mathrm{p}<$ 0.05. (F) Flow cytometry analysis of MNs with the indicated genotypes: WT, CTCF $(\Delta 5|6: 6| 7)$, and MAZ KO \& $\mathrm{CTCF}(\Delta 5|6: 6| 7)$. This plot is one representation of three biological replicates quantified in Fig. S6G. (G) RNA-seq MA plot of WT versus MAZ KO ESCs (left), and MNs (right) from three biological replicates. Differentially expressed genes are selected as p-value adjusted (padj) <0.05. Hox genes in 4 Hox clusters are colored based on their position with respect to the previously demonstrated CTCF-boundary in MNs. Hb9 is a MN marker. (H) Heat maps of CTCF and MAZ ChIP-seq read density in ESCs and MNs within a $4 \mathrm{~kb}$ window centered on the maximum value of the peak signal. (I to $\mathbf{J}$ ) Overlap of CTCF and MAZ binding sites in ESCs and MNs. ChIP-seq experiments are from one representative of two biological replicates. 
A
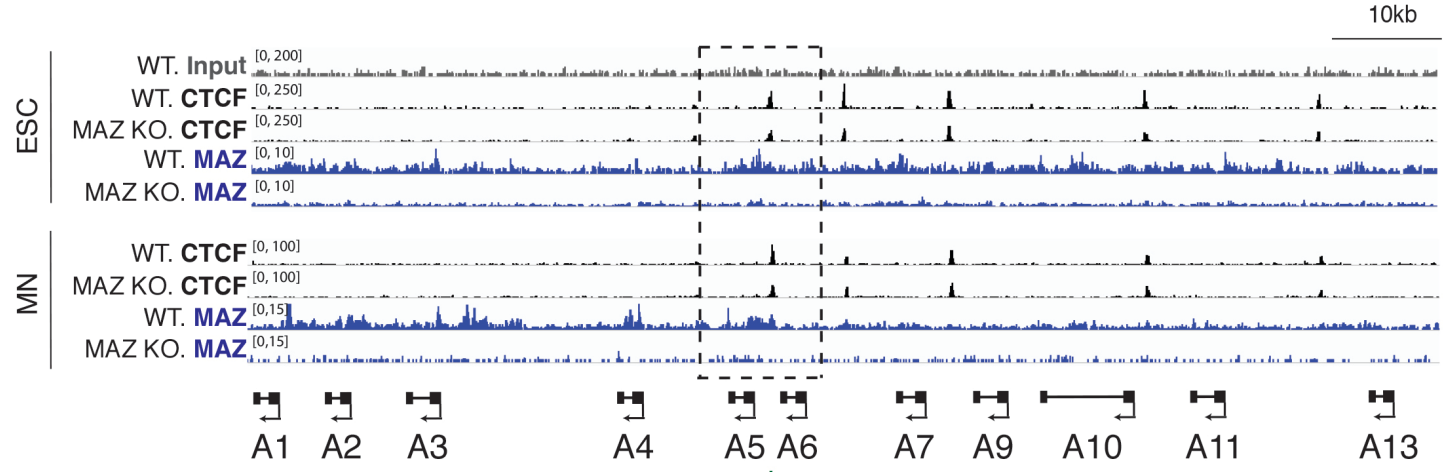

$\mathbf{B}$

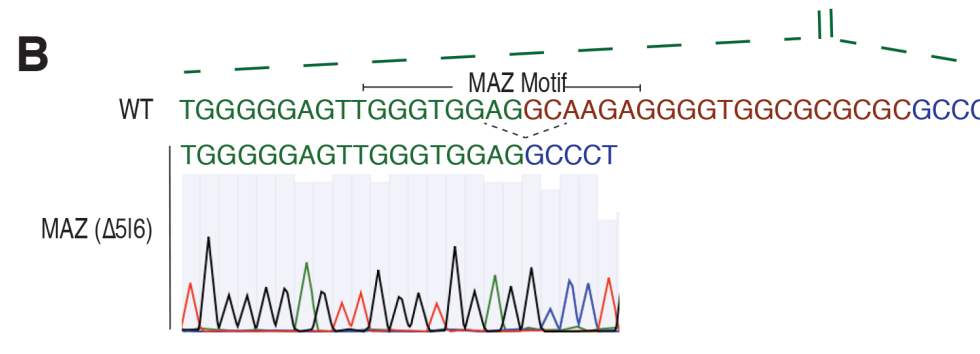

D

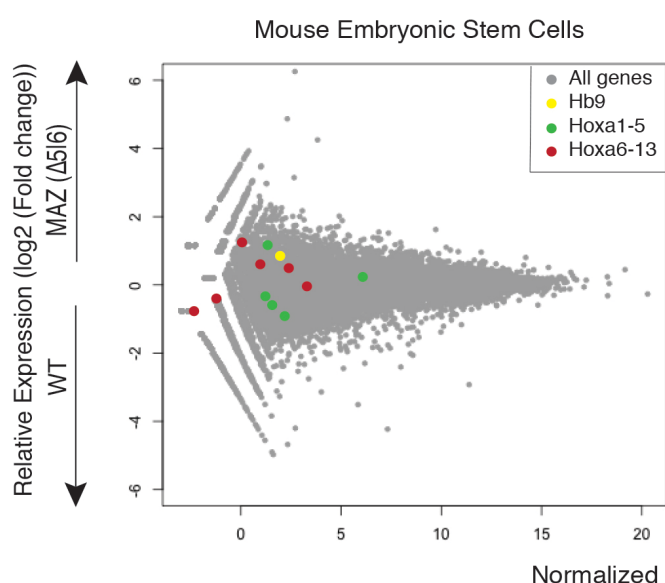

- - ${ }_{-1}$ - $-\mathbf{C}$

$\stackrel{\text { Anterior }}{\stackrel{\text { Posterior }}{\longleftrightarrow}}$

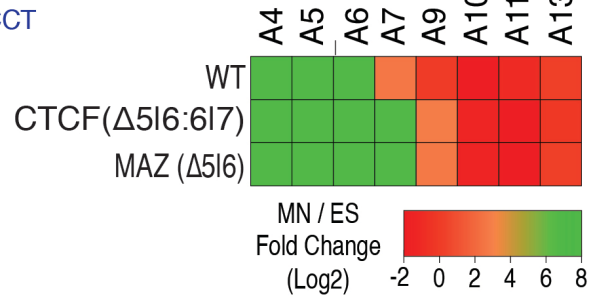

E
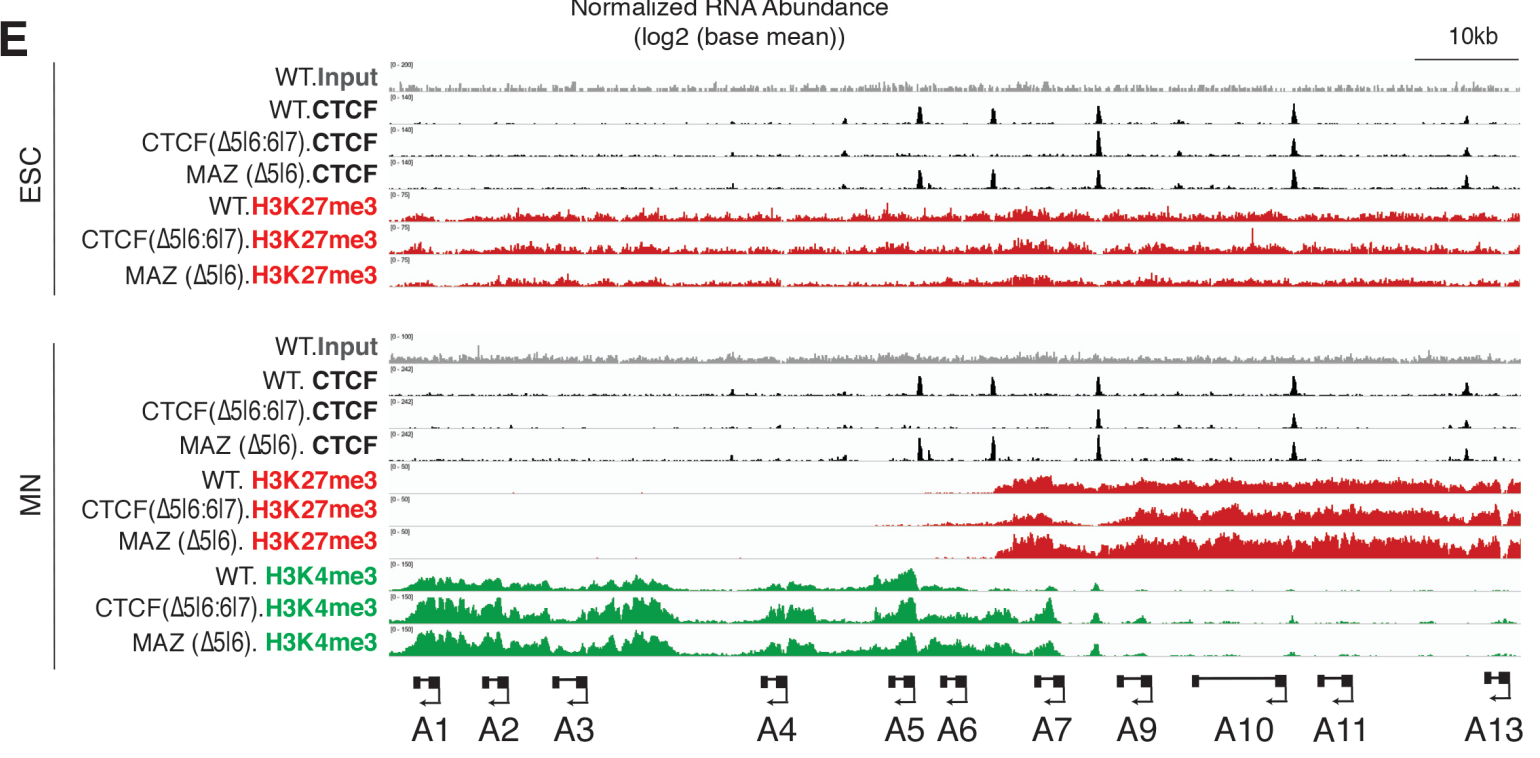
Fig. 3. Loss of MAZ binding site alters Hox gene expression pattern and chromatin domains at Hox clusters. (A) ChIP-seq for CTCF or MAZ in WT and MAZ KO ESCs and MNs in the HoxA cluster. (B) MAZ binding site deletion via CRISPR is depicted for the $5 \mid 6$ site at the HoxA cluster. (C) Heat map of relative gene expression in $\mathrm{WT}, \operatorname{CTCF}(\Delta 5|6: 6| 7)$, and $\operatorname{MAZ}(\Delta 5 \mid 6)$ at the HoxA cluster in MNs versus ESCs from three biological replicates. (D) RNA-seq MA plot of WT versus MAZ ( $\Delta 5 \mid 6)$ ESCs (left), and MNs (right) from three biological replicates. HoxA genes are colored based on their position with respect to the previously demonstrated CTCF boundary in MNs. $H b 9$ is a MN marker. (E) ChIP-seq for CTCF, and indicated histone modifications in WT, CTCF $(\Delta 5|6: 6| 7)$, and MAZ $(\Delta 5 \mid 6)$ ESCs and MNs in the HoxA cluster. ChIP-seq tracks are from one representative of two biological replicates for CTCF, one replicate for the histone modifications. 

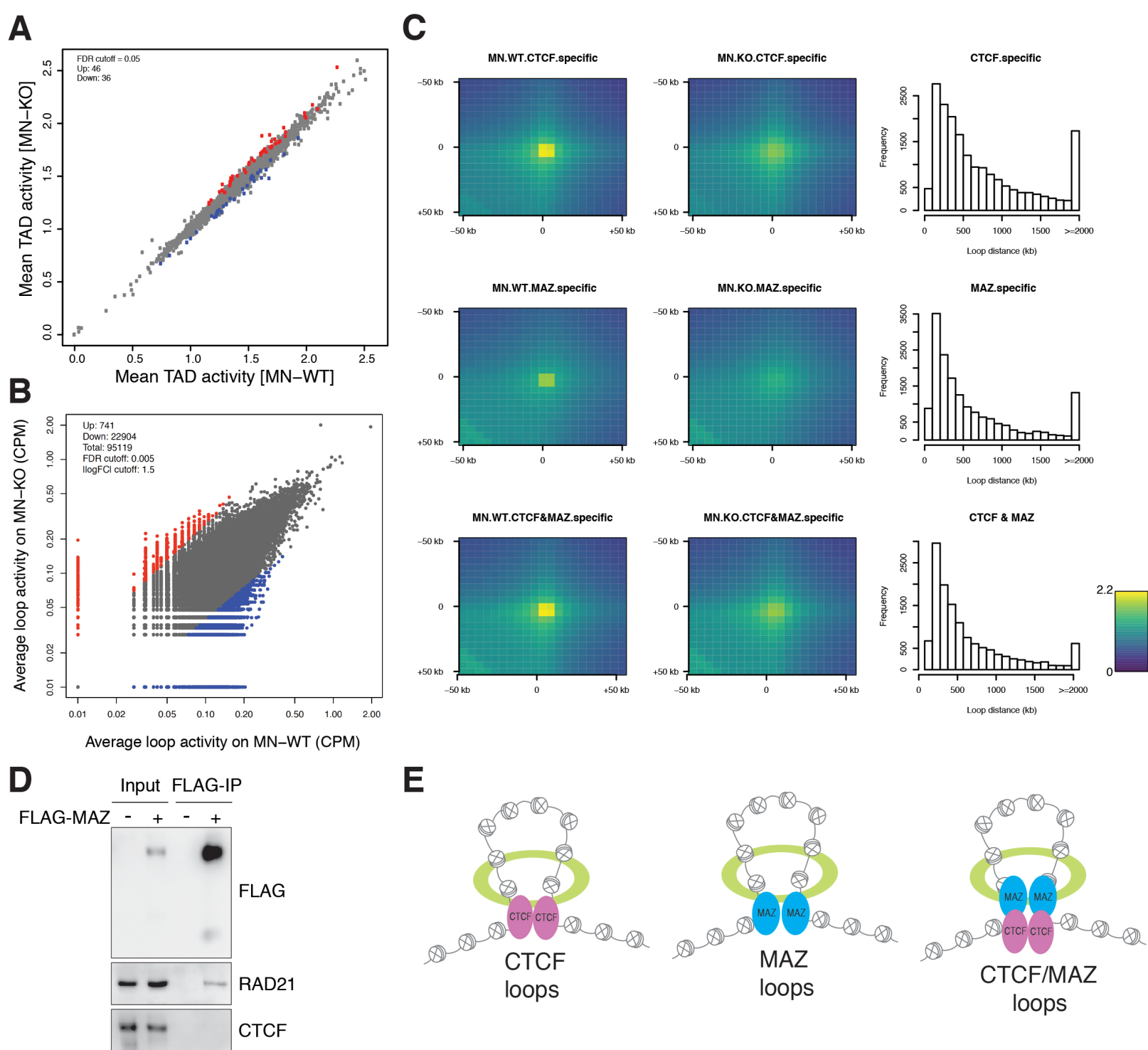

E
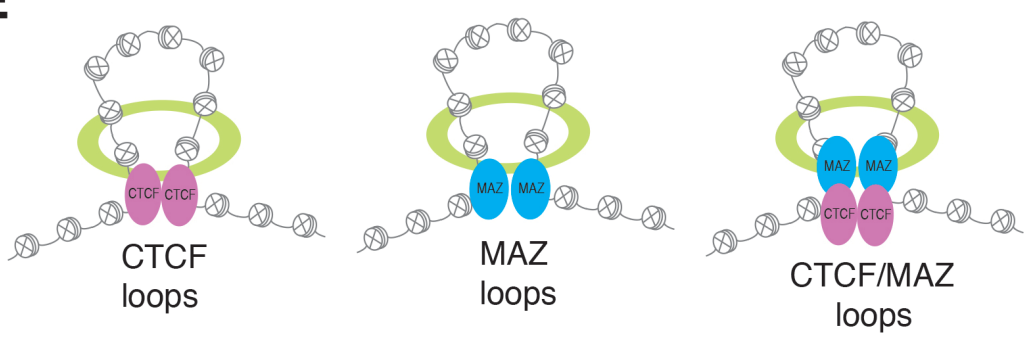

Fig. 4. Effect of MAZ on global genome organization. (A) Scatter plot showing differential intra-TAD activity in WT vs MAZ KO MNs (FDR cut-off=0.05). (B) Scatter plot showing differential loop activity in WT vs MAZ KO MNs (all loops, n=95119, FDR cut-off=0.005, | log

(Fold Change) $\mid$ cut-off=1.5, Up-regulated=741, Down-regulated: 22904). (C) Aggregate Peak Analysis (APA) of loops in WT vs MAZ KO MNs showing ChIP-seq signals of CTCF, MAZ, or both at any region covered by them. The resolution of APA is $5 \mathrm{~kb}$. Histograms showing the distribution of loop distance in MAZ KO compared to WT related to the binding level of ChIP- 
seq. (D) Western blot analysis of FLAG, RAD21, and CTCF upon FLAG-MAZ immunoprecipitation from nuclear extract of 293FT cells. (E) Proposed model for the effect of MAZ on loops via its interaction with cohesin (middle) or binding adjacent to CTCF and interaction with cohesin (right), in addition to existing loop-extrusion model of CTCF and cohesin (left, (44, 45)). 


\section{Supplementary Materials for}

\section{A CRISPR Screen Identifies Myc-associated Zinc Finger Protein (MAZ) as an Insulator Functioning at CTCF boundaries in Hox Clusters}

Havva Ortabozkoyun-Kara ${ }^{1,2}$, Pin-Yao Huang ${ }^{1,2},{\text { Hyunwoo } \mathrm{Cho}^{3,4,5}, \text { Varun Narendra }^{6} \text {, Gary }}^{*}$ Leroy $^{1,2}$, Jane A. Skok ${ }^{3}$, Aristotelis Tsirigos ${ }^{3,4,7}$, Esteban O. Mazzoni ${ }^{8}$ and Danny Reinberg ${ }^{1,2,}$

Correspondence to: Danny.Reinberg@nyulangone.org

\section{This PDF file includes:}

Materials and Methods

Figs. S1 to S14

Tables S1 to S3

Captions for Data S1 to S10

References (Full Reference List, 46 - 70)

Other Supplementary Materials for this manuscript include the following:

Supplementary Data S1 to S10 


\section{Materials and Methods}

\section{Cell Culture and Motor Neuron Differentiation}

E14 mouse embryonic stem cells (mESCs) were cultured in standard medium supplemented with LIF, and $2 \mathrm{i}$ conditions ( $1 \mathrm{mM}$ MEK1/2 inhibitor (PD0325901, Stemgent) and $3 \mathrm{mM}$ GSK3 inhibitor (CHIR99021, Stemgent)). For motor neuron (MN) differentiation, the previously described protocol was applied (16). Briefly, ESCs were differentiated into embryoid bodies in 2 days, and further patterning was induced with addition of $1 \mu \mathrm{M}$ all-trans-retinoic acid (RA, Sigma) and $0.5 \mu \mathrm{M}$ smoothened agonist (SAG, Calbiochem). Biological replicates stand for independent differentiation experiments performed. 293FT cells were cultured in standard medium as described in the manufacturer's protocol (Thermo Fisher Scientific).

\section{CRISPR Genome Editing}

sgRNAs were designed using CRISPR design tools in http://crispr.mit.edu/, currently available in https://benchling.com. All sgRNAs were cloned into SpCas9-2AGFP vector (Addgene: PX458) or into a lentiviral vector lentiGuide-puro (Addgene: 52963). The sgRNAs were transfected into mESCs using Lipofectamine 2000 (Invitrogen), as described before (16) or infected into a lentiCas9-blast (Addgene: 52962) expressing mESC clone. In the case of CRISPR knock-in cell lines, donor DNA ( $1 \mu \mathrm{l}$ of $10 \mu \mathrm{M}$ single stranded DNA oligo, or $3 \mu \mathrm{g}$ pBluescriptSK $(+)$ plasmid containing donor DNA) were transfected with $1 \mu \mathrm{g}$ px458-sgRNAs. Single clones from GFPpositive FACS sorted cells or puromycin (InvivoGen)-resistant cells were genotyped and confirmed by sequencing. When necessary, PCR products were further assessed by TOPO cloning, and sequencing to distinguish the amplified products of different alleles. The sequencing chromatograms were aligned in Benchling. All sgRNAs, donors, and genotyping primers are shown in Table $\mathbf{S 1 .}$ 


\section{Cell Line Generation}

\section{Hoxa5:a 7 dual reporter in WT and CTCF $(\Delta 5 \mid 6)$ backgrounds}

To generate Hoxa5:a 7 dual reporter cells, mESCs were sequentially targeted at Hoxa5 and Hoxa 7 loci, respectively. mESCs were initially transfected with sgRNA and donor pBluescriptSK $(+)$ plasmid for Hoxa5-P2A-mCherry cell line generation using Lipofectamine (Invitrogen). Hoxa5$m$ Cherry cell line was confirmed through genotyping, sequencing, and FACS analysis upon MN differentiation for the homozygous insertion of reporter. Next, the Hoxa5-mCherry cell line was transfected with sgRNA and donor pBluescriptSK $(+)$ plasmid for generation of the dual Hoxa5:a7 knock-in cell line, which was confirmed by genotyping, sequencing, and FACS analysis for the homozygous insertion of reporter. To demonstrate Hoxa7-P2A-GFP expression in MNs, CTCF binding sites at Hoxa5|6 and Hoxa6 7 were removed via sequential CRISPR genome editing using respective sgRNAs, generating $\mathrm{CTCF}(\Delta 5 \mid 6)$ and $\mathrm{CTCF}(\Delta 5|6: 6| 7)$ deletion lines in the Hoxa5:a 7 dual reporter background. For CRISPR library screen experiments, WT or CTCF $(\Delta 5|6: 6| 7)$ dual reporter lines were transduced with lentiCas9-blast (Addgene, 52962), and Cas9 expressing clones were obtained after selection with blasticidin (InvivoGen).

\section{FLAG-CTCF tagged cell line}

To generate the CTCF C-terminal FLAG-tagged cell line, E14 ESCs were targeted with sgRNA in SpCas9-2AGFP vector (Addgene: PX458) and single-stranded donor oligo at the CTCF locus and the cell line was confirmed by genotyping, sequencing, and western blot for FLAG-CTCF.

\section{MAZ KO cells}

WT or CTCF ( $\triangle 5|6: 6| 7)$ Hoxa5:a 7 dual reporter cells expressing Cas 9 were targeted with sgRNAs in lentiGuide-puro vector for Maz. Knock-out of Maz was confirmed by genotyping, sequencing, and western blot. 


\section{MAZ binding site deletions}

Hoxa5:a7 dual reporter cells were targeted with sgRNAs in SpCas9-2AGFP vector (Addgene: PX458) for MAZ binding sites at $\operatorname{HoxA}$, HoxD or HoxC cluster. Specific MAZ binding site deletions were confirmed by genotyping and sequencing.

\section{CRISPR Screens}

CRISPR genome-wide screens were done using methods described previously $(21,22)$. Briefly, GeCKO genome-wide pooled CRISPR libraries (Addgene: 1000000053) were amplified, and deep-sequenced to confirm sgRNA representations, as shown previously (21). A Cas 9 expressing Hoxa5:a7 ESC clone was transduced with the pooled lentiviral sgRNAs at a low multiplicity of infection (MOI) of $\sim 0.4$. The reporter ESCs were selected with puromycin, cultured for 7 days, and differentiated into MNs in 6 days, and sorted by FACS into two MN populations: (1) WT MNs ( $m$ Cherry positive/eGFP negative cells) and (2) CTCF-boundary disrupted MNs (double positive cells). During the screens, 300X and 1000X coverage was applied for genome-wide screens, and secondary screens, respectively. CRISPR libraries were prepared at each-time point and/or sorted population, and the relative sgRNA representation was assessed using next generation sequencing, as described previously $(21,22)$.

\section{Custom Library Construction for Secondary CRISPR Screens}

sgRNAs for custom library used in the secondary CRISPR screens were retrieved from a previously designed genome-wide mouse CRISPR knock-out pooled library (Brie) (34). When required for several genes, sgRNAs were designed by using the Broad Institute CRISPRko gRNA design tools (currently at https://portals.broadinstitute.org/gpp/public/analysis-tools/sgrnadesign). All sgRNAs in the custom library in Data $\mathbf{S 3}$ were synthesized as a pool by Twist 
Bioscience. The custom library was cloned into lentiGuide-puro vector, amplified, and verified in terms of representation of all constructs using the methods described previously (46).

\section{Flow Cytometry}

Cells were trypsinized, filtered, and stained with 4,6-diamidino-2-phenylindole (DAPI, Sigma) to eliminate dead cells during analysis of Hoxa5:a7 reporters in ESCs and MNs. Hoxa5:a7 dual fluorescent reporter cells in WT versus other backgrounds were assessed by using single color fluorescent reporters as controls in the same cell type as analyzed (i.e. MNs). Hb9-T2A-GFP reporter cells (not shown) were used as GFP control in MNs. For cell cycle analysis, ESCs were fixed in 75\% Ethanol, and DNA was stained with Propidium iodide (Thermo Fisher Scientific) after RNase A (Thermo Fisher Scientific) treatment. FlowJo 8.7 was used for all FACS analysis.

\section{Expression Analysis}

RNA was purified from cells with RNAeasy Plus Mini kit (Qiagen), and reverse transcription was performed on $1 \mu \mathrm{g}$ RNA by using Superscript III (Life Technologies) and random hexamers (Thermo Fisher Scientific). RT-qPCRs were performed in replicates on $100 \mathrm{ng}$ cDNA using PowerUp SYBR Green Master Mix (Thermo Fisher Scientific). The primers are listed in Table S2. For RNAseq analysis, $1 \mu \mathrm{g}$ RNA was used to prepare ribominus RNAseq libraries according to the manufacturer's protocols by the NYU Genome Technology Center.

\section{Chromatin Immunoprecipitation Sequencing}

ChIP-seq experiments were performed as described (47) [see details about ESC fixation (47), and MN fixation in (16)]. Briefly, cells were fixed with $1 \%$ formaldehyde, nuclei were isolated, and chromatin was fragmented to $\sim 250 \mathrm{bp}$ in size using a Diagenode Bioruptor. ChIP was performed by using antibodies listed in Table S2. Chromatin from Drosophila (1:100 ratio to ESC or MN derived chromatin), and Drosophila-specific H2Av antibody were used as spike-in control in each 
sample. For ChIP-seq, libraries were prepared as described (16) using 1-30 ng of immunoprecipitated DNA. ChIP-qPCRs were performed with PowerUp SYBR Green Master Mix (Thermo Fisher Scientific) and detected by the Stratagene Mx3005p or QuantStudio 5 (Thermo Fisher Scientific) instrument. All ChIP-qPCR primers are listed in Table S2.

\section{Preparation of HiC samples}

Cells were harvested, and $1 \mathrm{M}$ cells were fixed in 2\% formaldehyde (Fisher Chemical) according to the ARIMA-HiC protocol. Samples were prepared and sequenced according to the manufacturer's protocol by NYU Genome Technology Center.

\section{Cellular Fractionation and Immunoprecipitation}

All cellular fractionation and immunoprecipitation experiments were performed at $4^{\circ} \mathrm{C}$ or on ice with buffers containing $1 \mu \mathrm{g} / \mathrm{ml}$ Pepstatin, $1 \mu \mathrm{g} / \mathrm{ml}$ Aprotonin, $1 \mu \mathrm{g} / \mathrm{ml}$ Leupeptin, $0.3 \mathrm{mM}$ PMSF, $10 \mathrm{mM}$ Sodium fluoride, and $5 \mathrm{mM}$ Sodium orthovanadate. For FLAG affinity purification from native chromatin (native ChIP-mass spectrometry), nuclear extracts in ESCs and MNs were prepared using Buffer A and Buffer C as described (48). Cytosolic fraction was removed by Buffer A (10 mM Tris, $\mathrm{pH} 7.9,1.5 \mathrm{mM} \mathrm{MgCl} 2,10 \mathrm{mM} \mathrm{KCl}$, and $0.5 \mathrm{mM}$ dithiothreitol (DTT)). The pellet was resuspended in Buffer C (20 mM Tris, $\mathrm{pH} 7.9,25 \%$ glycerol, $420 \mathrm{mM} \mathrm{NaCl}, 1.5 \mathrm{mM} \mathrm{MgCl}$, $0.2 \mathrm{mM}$ EDTA, and $0.5 \mathrm{mM}$ DTT), and incubated $1 \mathrm{hr}$ to obtain nuclear extract. After removing nuclear extract, the remaining nuclear pellet was solubilized by benzonase (Millipore) digestion in a buffer containing $50 \mathrm{mM}$ Tris, $\mathrm{pH} 7.9$ and $2 \mathrm{mM} \mathrm{MgCl}_{2}$. For FLAG affinity purification from native chromatin and mass spectrometry, $20 \mathrm{mg}$ nuclear pellet was incubated with $200 \mu \mathrm{l}$ FAG M2 beads in BC250 overnight and washed six times with BC250 containing $0.05 \%$ NP40, as described (49). Two elutions were performed with $0.5 \mathrm{mg} / \mathrm{ml}$ FLAG peptide in BC50 (without any protease inhibitors) with rotation at $4^{\circ} \mathrm{C}$ for $2 \mathrm{hr}$ for a total of $4 \mathrm{hr}$. The eluate was sent for mass 
spectrometry to the Rutgers Mass Spectrometry Center and analyzed by liquid chromatographymass spectrometry (LC-MS/MS). Peptide counts are shown for the native ChIP-mass spectrometry experiments in Data S2.

For FLAG affinity purification from crosslinked chromatin (crosslinked ChIP-mass spectrometry), a modified version of a previously reported protocol was applied $(31,32)$. Briefly, cells were crosslinked and sonicated as described above for ChIPseq except with a larger fragment size to include $~ 3-5$ nucleosomes. $3 \mathrm{mg}$ chromatin was used for FLAG affinity purification, and FLAG elutions were performed after stringent washes as described (31), but excluding the second step in the protocol wherein DNA is biotinylated. After de-crosslinking, samples were sent for mass spectrometry to the Rutgers Mass Spectrometry Center and analyzed by liquid chromatographymass spectrometry (LC-MS/MS).

For extraction in 293FT cells, C $\beta$ F expression vectors containing cDNAs for CTCF (mouse) or MAZ (mouse) were transfected into 293FT cells using PEI, and nuclei was prepared by using TMSD and BA450 buffers as described before (50, 51). Briefly, TMSD buffer (20 mM HEPES, 5 $\mathrm{mM} \mathrm{MgCl}_{2}, 85.5 \mathrm{~g} / \mathrm{L}$ Sucrose, $25 \mathrm{mM} \mathrm{NaCl}$, and $1 \mathrm{mM}$ DTT) was used for cytosol removal, and nuclear extraction was done in BA450 buffer (20 mM HEPES, $450 \mathrm{mM} \mathrm{NaCl}, 5 \%$ glycerol, and 0.2 mM EDTA). FLAG affinity purification and FLAG peptide elution were performed similarly in the nuclear fraction.

\section{Library Construction}

All libraries were prepared according to the manufacturer's instructions (Illumina). CRISPR libraries were prepared by performing two-step PCRs as described (22). Briefly, sgRNAs were amplified from genomic DNA by keeping the coverage maintained throughout the screens (300X for GeCKO v2 library, 1000X for custom library in secondary screens) and performing secondary 
amplifications by using Phusion polymerase (New England Biolabs) to attach Illumina adaptors (shown in Table S3). ChIPseq libraries were prepared as described (16). RNAseq libraries were prepared using KAPA library preparation kits, and HiC libraries were prepared according to the ARIMA standard HiC protocols by the NYU Genome Technology Center.

\section{Data Analysis}

\section{CRISPR screens}

MaGeCK tools were used for all primary and secondary CRISPR screen analysis (26, 27). Genome-wide screens with GeCKO v2 library A (3sgRNAs/gene) and GeCKO v2 library B (3sgRNAs/gene) were analyzed together in total populations of ESCs and MNs to identify essential/differentiation related genes (negative selections). The analysis was done separately for library A (2 screens) and library B (2 screens) in sorted MN populations to identify genes affecting CTCF boundary function (positive selection). PANTHER database was used for Gene Ontology (GO) analysis (52). To generate Venn diagrams in CRISPR screens, web-tools at http://genevenn. sourceforge.net were used.

\section{RNAseq}

RNA-seq data was analyzed as described (16). Briefly, sequence reads were mapped to mm10 reference genome with Bowtie 2 (53) and normalized differential gene expression was obtained with DEseq (R package) (54). Relevant expression and p-values are listed for differentially expressed genes in Data S7-S10. PANTHER database was used for Gene Ontology (GO) analysis (52).

\section{ChIPseq}

ChIPseq experiments were analyzed as before (47). In brief, sequence reads were mapped to mm10 reference genome with Bowtie 2 using default parameters (53). After normalization with the spike- 
in Drosophila read counts, normalized ChIP-seq read densities were visualized in Integrative Genomics Viewer (IGV) (55). Heat maps were generated using deepTools in R (56). 'ChIPpeakAnno' package from Bioconductor (57) was used to draw Venn diagrams to visualize the overlap among ChIP-seq samples. The replicates were assessed similarly by visualizing at IGV and generating heat maps. ChIPseq "bed" file coordinates were converted into "fasta" by using fetch sequences tool within Regulatory Sequence Analysis Tools (RSAT) (58), and MEME was used for motif analysis of MAZ in ESCs and MNs (59).

\section{HiC}

All samples were prepared in two biological replicates. All Hi-C data were analyzed by the hicbench platform (60). Throughout our comprehensive analysis, the following operations were done using hic-bench. Internally, bowtie 2 (61) was used to align the paired reads using mm10 reference genome and only the read pairs uniquely mapped to the same chromosome with the mapping quality $\geq 20$ and the pair distance $\geq 25 \mathrm{~kb}$ were used. Then, the interaction matrix was tabulated by reading the coordinates of aligned reads in 20 kilobase bins. To ensure that each interaction bin showed equal visibility, the iterative correction method (62) was used to normalize the bins.

For the compartment analysis, the Hi-C interaction bins were divided into A and B compartments using the first principal component values from HOMER's runHiCpca $(63,64)$. Using hic-bench, the compartment changes from comparison of two cell types for the bins in the interaction matrix were visualized by the stacked bar plot.

Topologically associated domains (TADs) were defined as shown $(60,65)$ with the insulating window of $500 \mathrm{~kb}$. The boundaries of TADs were called from the boundary score, using the "ratio" method defined (60), wherein each TAD boundary had a noticeably lower boundary score than the neighboring region. The score was calculated for each 20 kilobase bin using the window size of 
$250 \mathrm{~kb}, 500 \mathrm{~kb}$, and $1000 \mathrm{~kb}$. In the principal component analysis to distinguish the differences, the boundary score for every replicate and cell type was combined, quantile normalized, and plotted. Then, for each TAD, the magnitude of intra-TAD "activity" was defined as in (65). The cutoff for significantly differential TADs was Benjamini-Hochberg corrected Q value of 0.05 and no cutoff for the fold change.

Significantly enriched chromatin loops were called using FitHi-C 2 (66) with default parameters. To characterize the loops by the level of CTCF and MAZ ChIP-seq level, aggregate peak analysis (APA) software (67) was used to show the averaged profile. The genome sequence that matched the transcription factor motifs of mouse CTCF and MAZ from the Catalog of Inferred Sequence Binding Preferences (CIS-BP) (68) was found from PWMScan (69). Visualization of Hi-C and associated ChIP-seq data was made with pyGenomeTracks (70). 
A

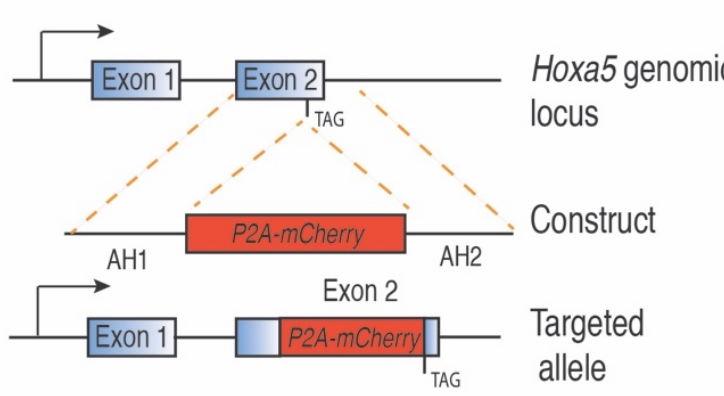

B

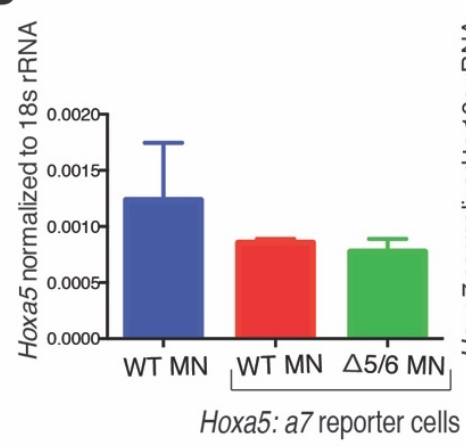

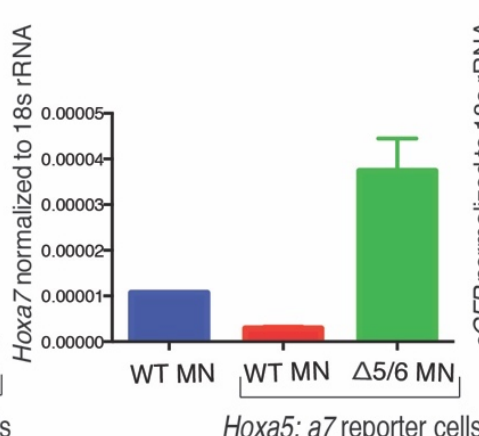

Hoxa5: a7 reporter cells
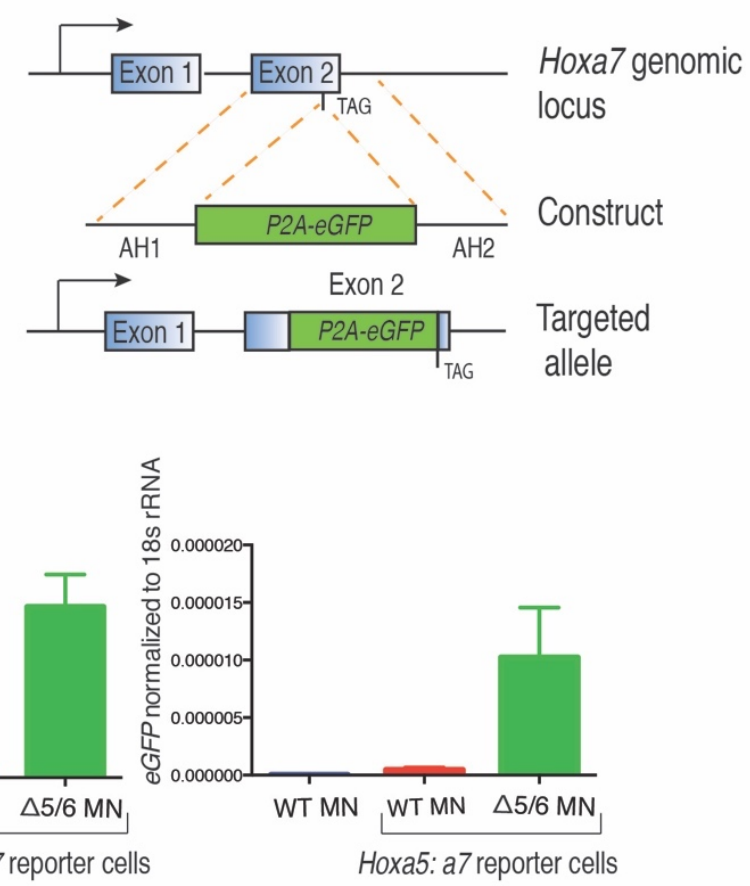

C

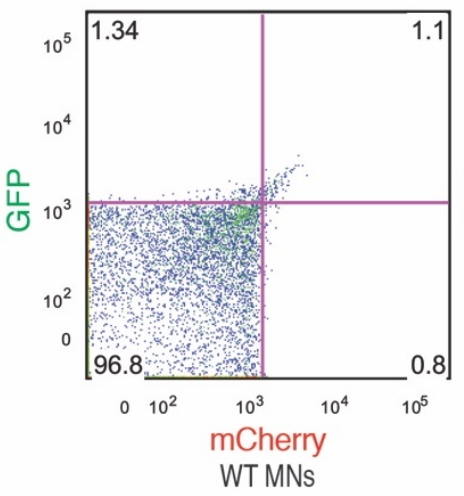

D

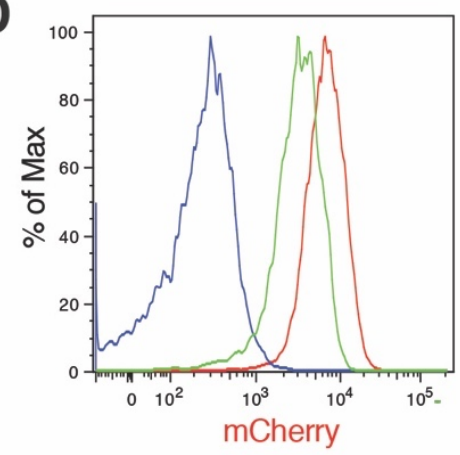

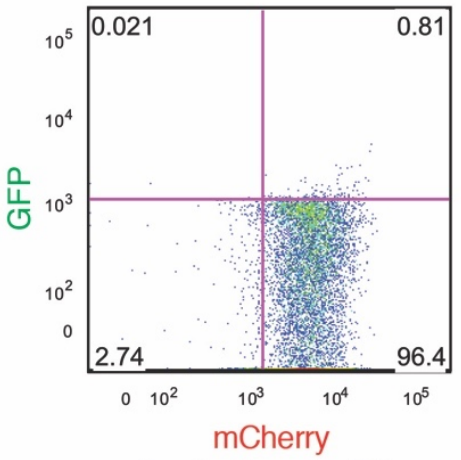

Hoxa5: a7 reporter MNs

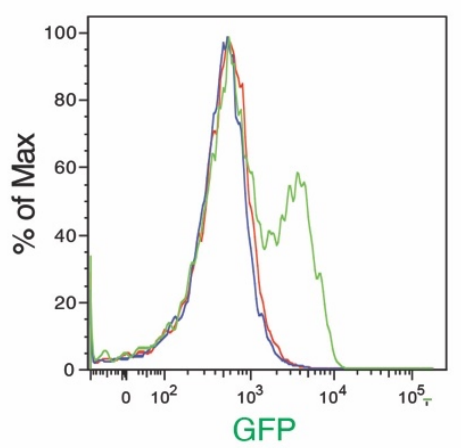

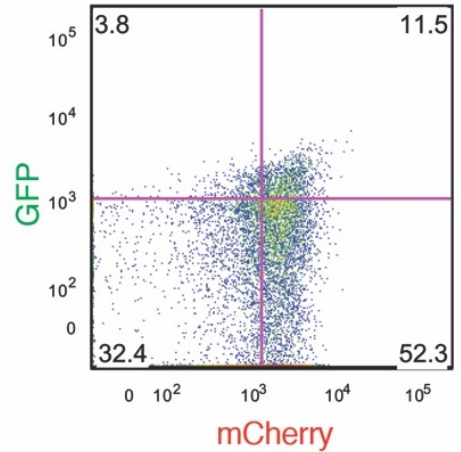

CTCF $\triangle 5 / 6: 6 / 7$

Hoxa5: a7 reporter MNs

$\square \operatorname{CTCF}(\Delta 516: 617)$ reporter

WT

WT Hoxa5:a7 reporter 
Fig. S1. A dual-fluorescent reporter Hoxa5:a 7 mESC line can be used to assess disruption of a functional CTCF boundary in MNs

A. Strategy for generating the Hoxa5:a7 reporter mESC line via CRISPR. AH1, AH2: arm of homology 1, 2. B. RT-qPCR signal for Hoxa5, Hoxa7, and eGFP normalized to 18s ribosomal RNA in WT MNs, Hoxa5:a 7 reporter MNs, and $\mathrm{CTCF}(\Delta 5 \mid 6)$ Hoxa5:a 7 reporter MNs. Error bars indicate the standard deviation across two biological replicates. MNs were obtained through in vitro differentiation of mESCs. C. FACS data showing mCherry and eGFP reporter expression in WT MNs (left), Hoxa5:a7 reporter MNs (middle), and $\mathrm{CTCF}(\triangle 5|6: 6| 7)$ Hoxa5:a7 reporter MNs (right). These plots are one representative of three biological replicates. Percentage of positive population in each quadrant is indicated. D. Flow cytometry analysis of mCherry and eGFP reporter expression in MNs with the indicated genotypes: WT, Hoxa5:a7 reporter, and $\operatorname{CTCF}(\Delta 5|6: \Delta 6| 7)$ reporter (see Fig. S6 for RT-qPCR of Hox genes). These plots are one representative of three biological replicates. $\Delta 5 \mid 6$ refers to CTCF binding site deletion between Hoxa5-6. $\Delta 5|6: 6| 7$ stands for 2 CTCF binding site deletions. 
A

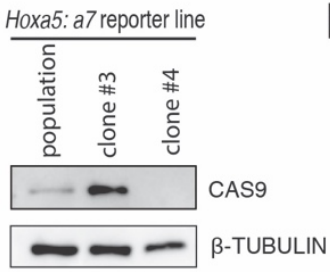

B

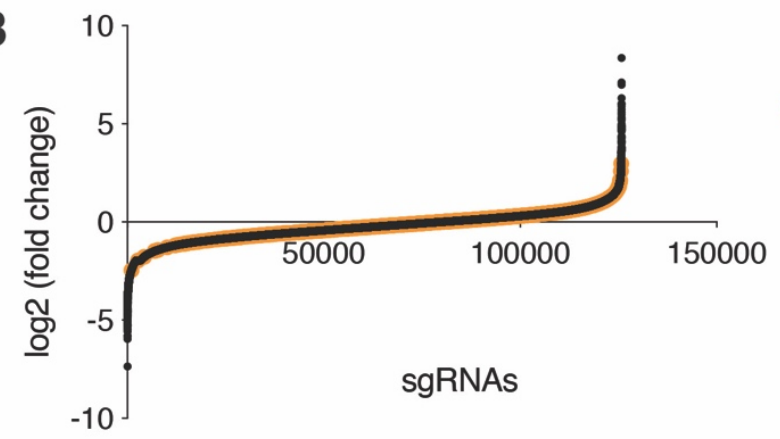

- all sgRNAs

- Nontargeting sgRNAs

C

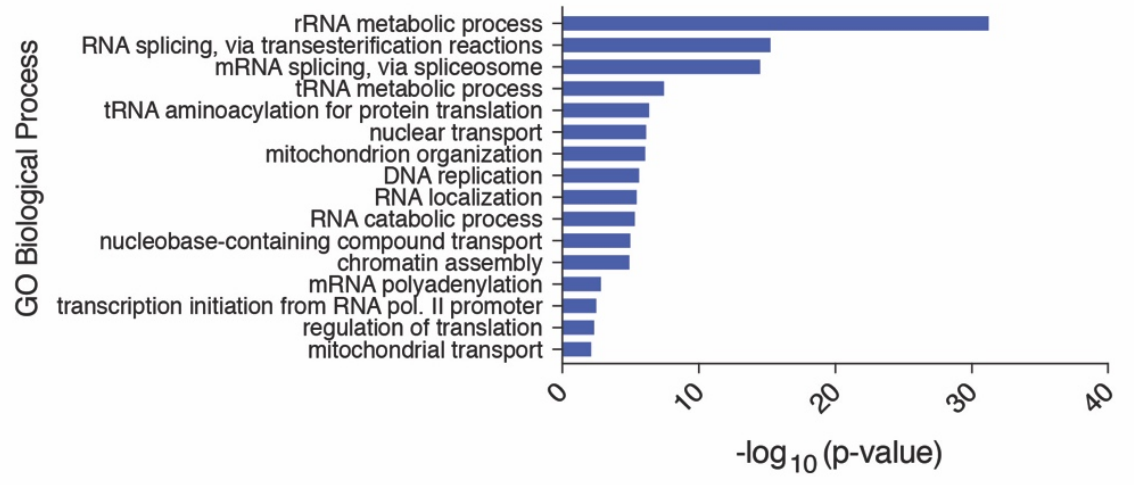

D

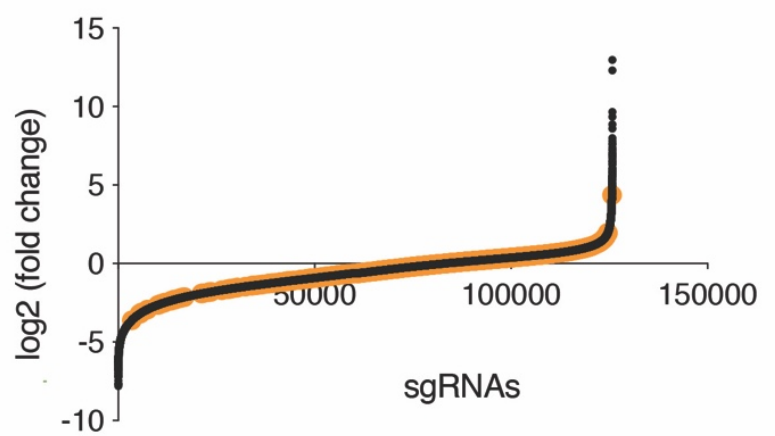

- all sgRNAs

- Nontargeting sgRNAs

E

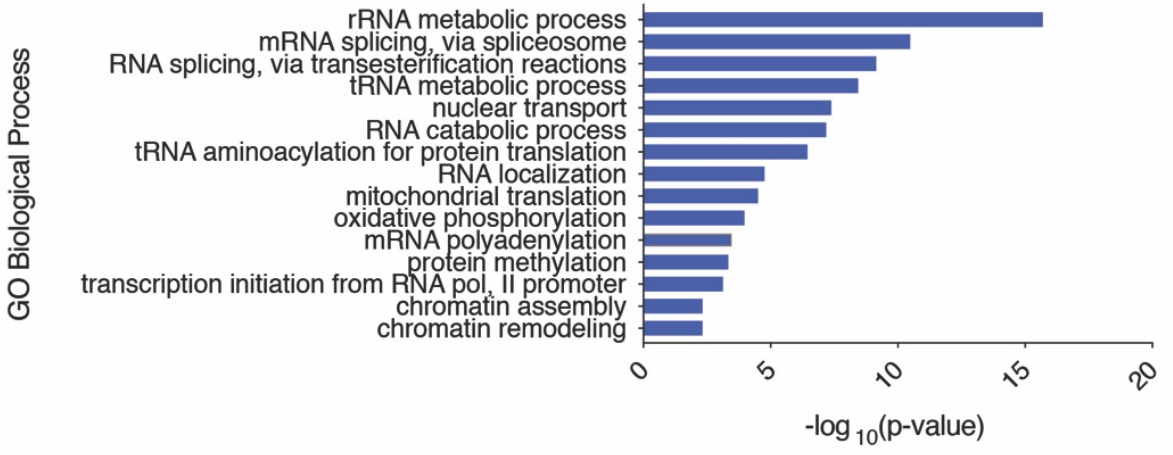


Fig. S2. Genome-wide CRISPR KO screen shows selective loss of essential genes in ESCs, and MNs

A. CAS9 protein expression in dual reporter mESCs transduced with Cas9 lentivirus and selected with blasticidin. $\beta$-TUBULIN acts as a loading control. B. Fold change of sgRNAs in the primary screens comparing ESCs to plasmid library. C. Gene Ontology (GO) analysis of biological processes in negatively selected genes in ESCs compared to plasmid library $(\mathrm{FDR}<0.05)$. D. Fold change of sgRNAs in the primary screens comparing MN population to ESC population. E. GO analysis of biological processes in negatively selected genes in MNs compared to ESCs $(\mathrm{FDR}<0.05)$ 
A

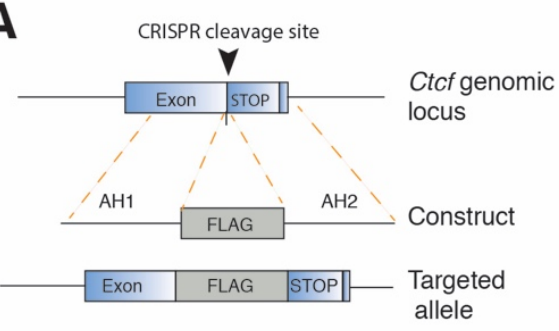

D
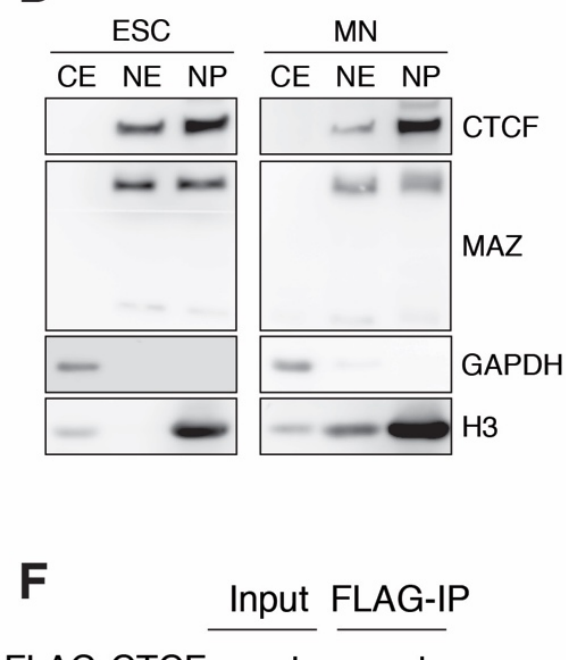

FLAG-CTCF - $+\quad-\quad+$

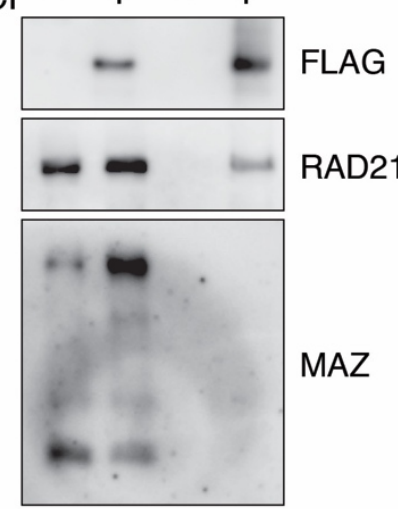

B

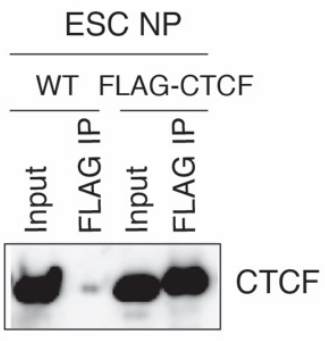

E

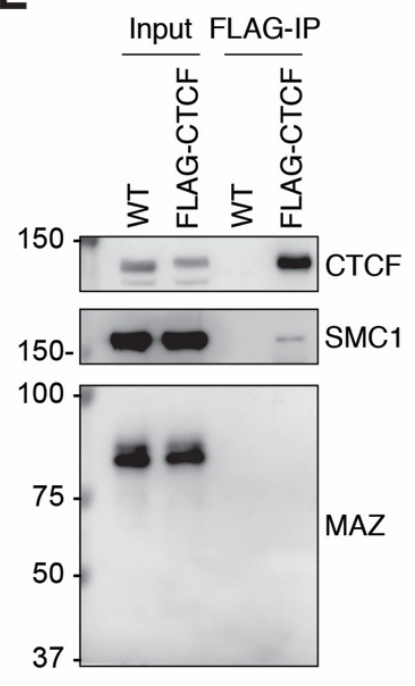

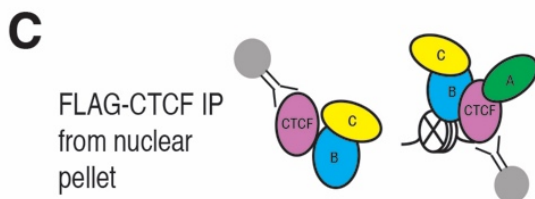

\begin{tabular}{|c|c|c|c|c|}
\hline \multirow{2}{*}{ Genes } & \multicolumn{2}{|c|}{ ESCs } & \multicolumn{2}{c|}{ MNs } \\
\cline { 2 - 5 } & Control & CTCF IP & Control & CTCF IP \\
\hline Ctcf & 1 & 1782 & 3 & 1082 \\
\hline Chd4 & 10 & 271 & & 120 \\
\hline Ddx5 & 11 & 288 & 17 & 237 \\
\hline Safb & & 210 & & 178 \\
\hline Trim28 & 21 & 301 & 1 & 116 \\
\hline Polr2a & 2 & 167 & & 98 \\
\hline Fam208a & & 125 & & 16 \\
\hline Smc1a & 1 & 119 & & 55 \\
\hline Rbmxl1 & 10 & 221 & 6 & 179 \\
\hline Smc3 & & 119 & & 55 \\
\hline Dnmt3a & & 120 & & 69 \\
\hline Ddx3x & & 117 & 2 & 98 \\
\hline Smarca5 & 3 & 163 & & 51 \\
\hline Adnp & & 105 & & 70 \\
\hline Polr2b & 1 & 125 & & 75 \\
\hline Hdac1 & 8 & 88 & & 52 \\
\hline Safb2 & & 97 & & 57 \\
\hline
\end{tabular}

Bait

Cohesin related

Other known interactors, and co-occupying proteins

Fig. S3. Identification of proteins co-localizing with CTCF via native ChIP-MS in ESCs and MNs

A. FLAG-tag integrated at the C-terminus of CTCF via CRISPR genome editing. AH1, 2: arm of homology 1, 2. B. FLAG pull-down followed by CTCF western blot in benzonase solubilized nuclear pellet (NP) of ESCs. C. Native FLAG-CTCF IP in ESCs and MNs results in identification of known CTCF interactors, and novel proteins. The mean peptide counts from two biological 
replicates of FLAG-CTCF IPs were normalized to the control FLAG IP from untagged cells. Candidates filtered from the top of the list are shown (see Data $\mathbf{S 2}$ for all). D. Western blot analysis of CTCF and MAZ in different cellular fractions in mESCs and MNs. CE: cytoplasmic extract, NE: nuclear extract, NP: nuclear pellet. E. Western blot analysis of CTCF, SMC1, and MAZ upon FLAG-CTCF immunoprecipitation from nuclear pellet of mESCs. F. Western blot analysis of FLAG, RAD21, and MAZ upon FLAG-CTCF immunoprecipitation from nuclear extract of 293FT cells. 
A

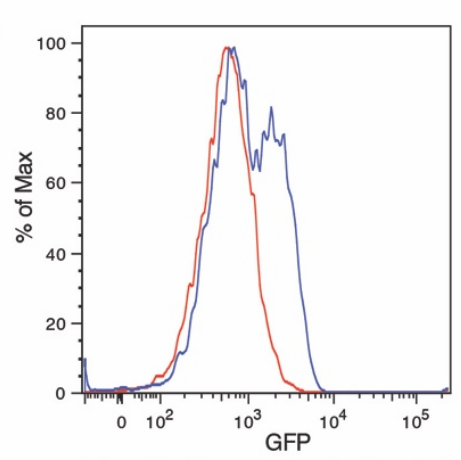

Lentiviral library expressing Hoxa5:a7 reporter

WT Hoxa5:a7 reporter

C

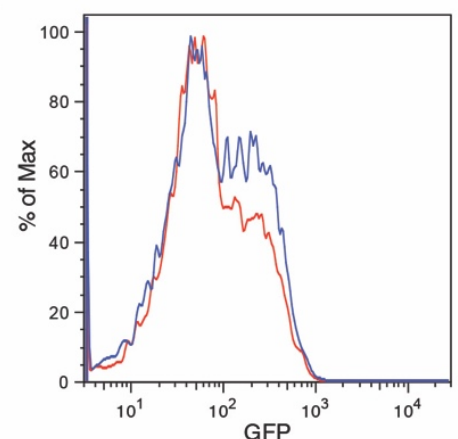

B

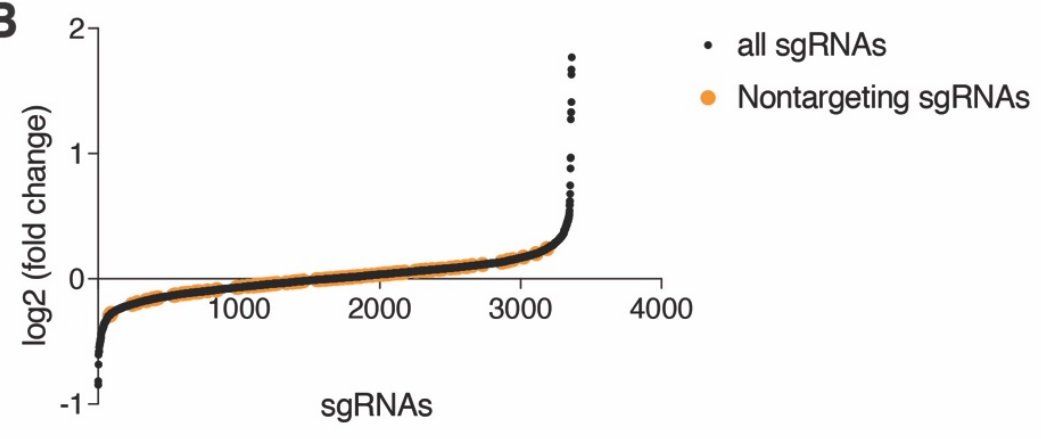

D

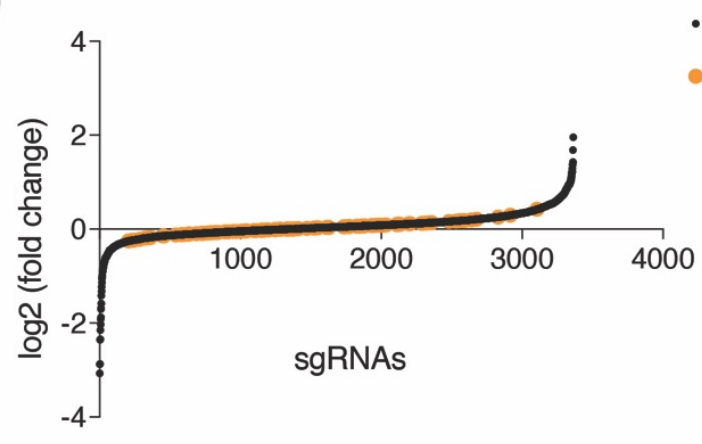

- all sgRNAs

- Nontargeting sgRNAs

Lentiviral library expressing $\operatorname{CTCF}(\Delta 516: 617)$ reporter $\operatorname{CTCF}(\Delta 516: 617)$ reporter

E

\section{Genome-wide Screens}

Sub-Library A (2X)

- Top 50 genes in each screen

Sub-Library B (2X)

- Top 50 genes in each screen

Common candidates to A-B screens

- 200 genes

\section{Biochemical Studies}

Candidates from

CTCF-mass spectrometry

from native/crosslinked

chromatin ( 350 genes)

\section{Secondary Screen}

Custom Library (3368 sgRNAs)

- 4 sgRNAs/gene

- 817 genes including possible controls

(i.e. CTCF, cohesin components...etc.)

- 100 nontargeting controls

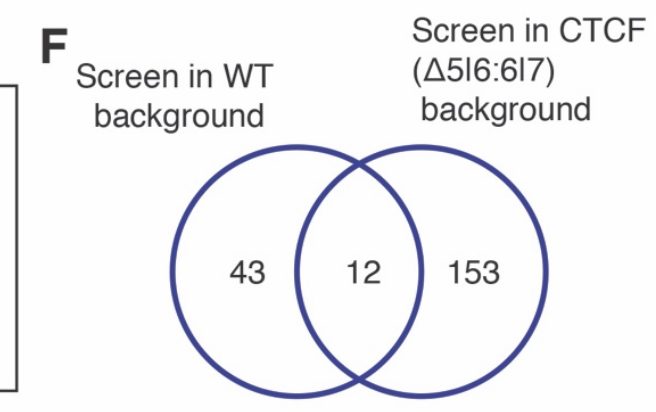


Fig. S4. Secondary loss-of-function screen at the CTCF boundary in the HoxA cluster results in a small list of candidates

A. FACS analysis of GFP expression in lentivirus library expressing MNs in WT background (with intact CTCF binding sites) versus untransduced WT MNs. B. Fold change of sgRNAs in the secondary screen performed in WT background. C. FACS analysis of GFP expression in lentivirus library expressing $\mathrm{MNs}$ in $\mathrm{CTCF}(\Delta 5|6: 6| 7)$ background versus untransduced $\mathrm{CTCF}(\Delta 5|6: 6| 7)$ MNs. D. Fold change of sgRNAs in the secondary screen performed in $\operatorname{CTCF}(\Delta 5|6: 6| 7)$ background. E. Schematic of candidate selection for the secondary loss-of-function screens. F. Venn diagram depicting overlap of secondary genetic screens in WT versus CTCF( $\Delta 5|6: 6| 7)$ background. 
bioRxiv preprint doi: https://doi.org/10.1101/2020.08.25.267237; this version posted August 26, 2020. The copyright holder for this preprint (which was not certified by peer review) is the author/funder. All rights reserved. No reuse allowed without permission.

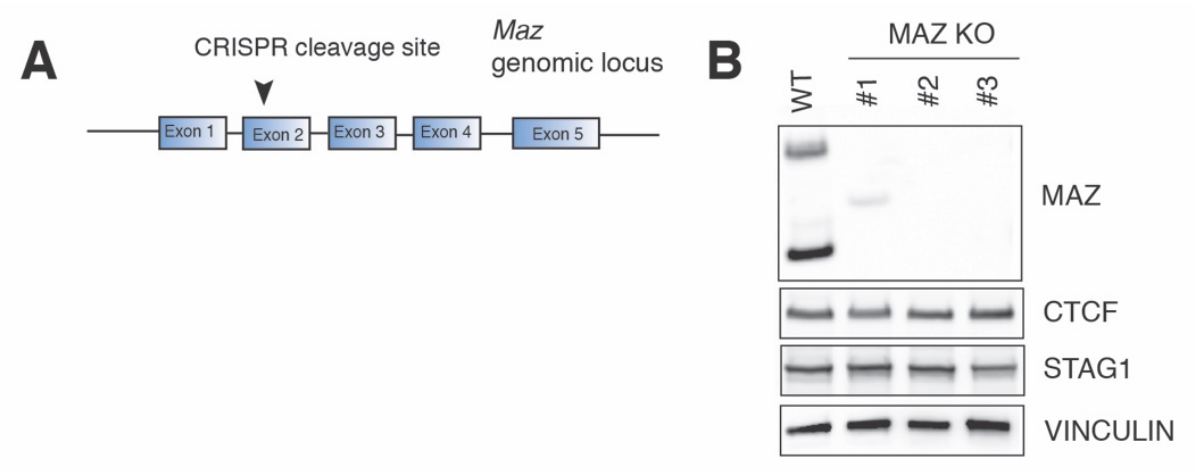

RNA metabolic process nucleic acid metabolic process

RNA processing mRNA processing rRNA metabolic process mRNA splicing, via spliceosome maturation of SSU-rRNA nuclear transport hexose metabolic process rRNA processing mitochondrion organization heterocycle biosynthetic process cellular amino acid metabolic process nucleobase-containing compound biosynthetic process purine ribonucleotide biosynthetic process RNA 3'-end processing negative regulation of gene expression organophosphate metabolic process maturation of SSU-rRNA from tricistronic rRNA transcript

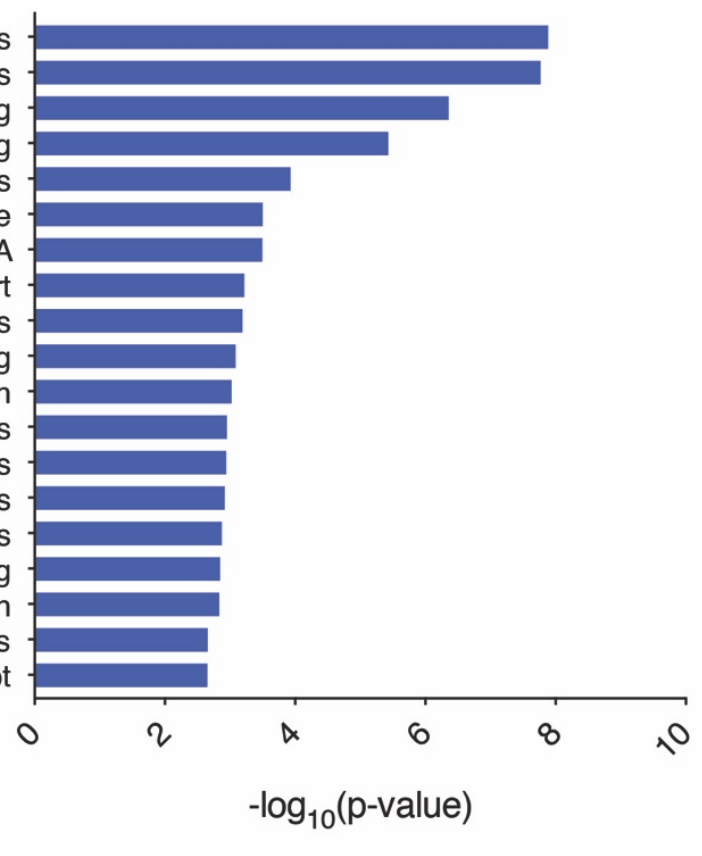

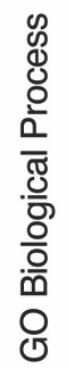

anterior-posterior pattern specification regionalization regulation of cellular component organization supramolecular fiber organization actin cytoskeleton organization actin filament-based process axon guidance organic hydroxy compound transport regulation of cell cycle regulation of cell shape regulation of organelle organization embryonic organ development regulation of locomotion regulation of mitotic nuclear division Wnt signaling pathway cell morphogenesis cellular component morphogenesis regulation of cell morphogenesis developmental growth system development regulation of nuclear division vesicle fusion to plasma membrane

0

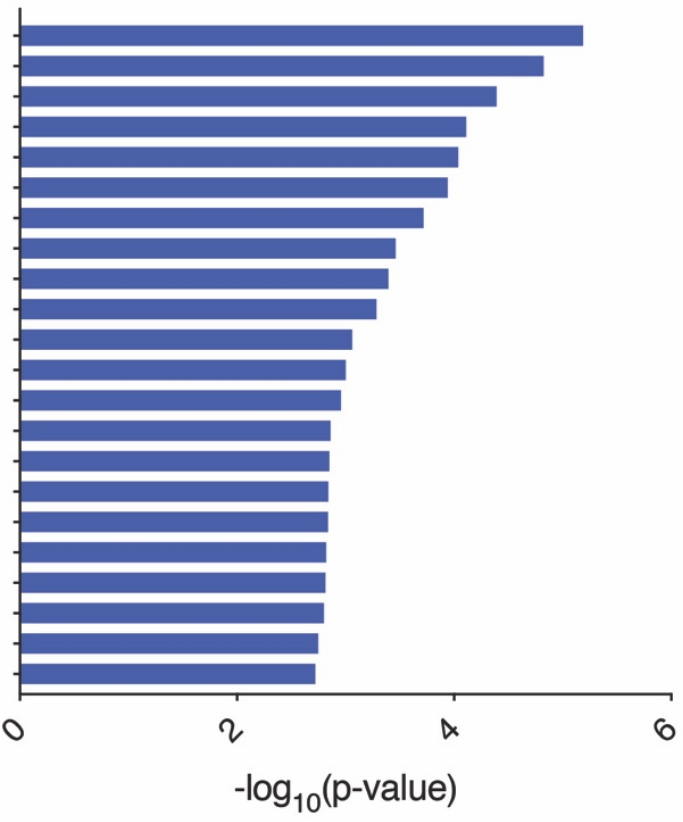


Fig. S5. Developmental and neuronal processes are enriched upon loss of $\mathrm{Maz}$ in motor neurons

A. The strategy of MAZ KO mESC line generation via CRISPR. B. Western blot analysis of indicated proteins in WT and MAZ KO ESCs. C. GO analysis of biological processes in differentially expressed genes in MAZ KO ESCs compared to WT ESCs. D. GO analysis of biological processes in differentially expressed genes in MAZ KO MNs compared to WT MNs. 
A

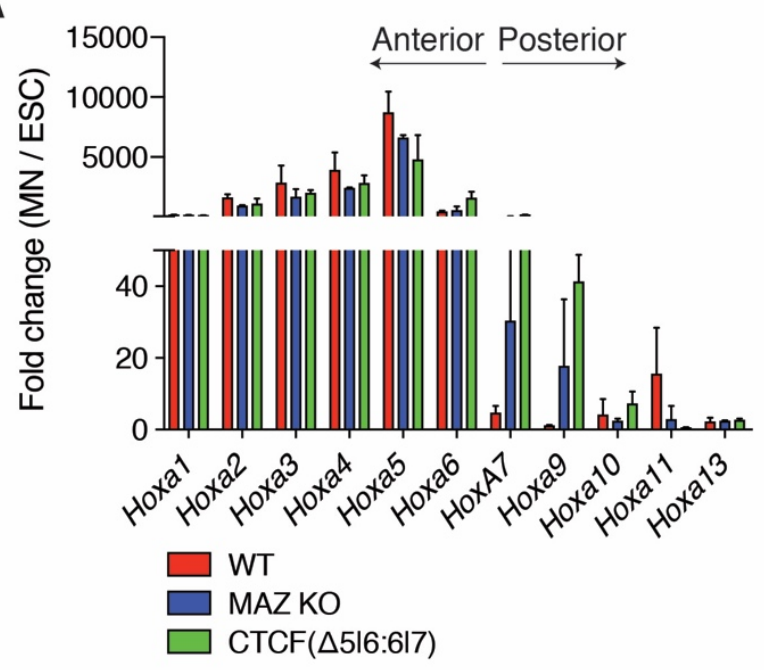

C

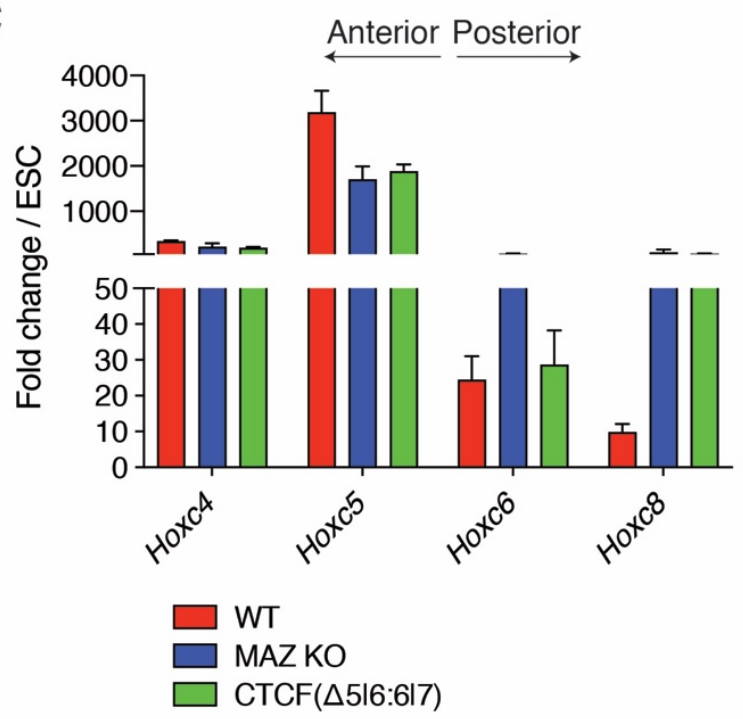

E

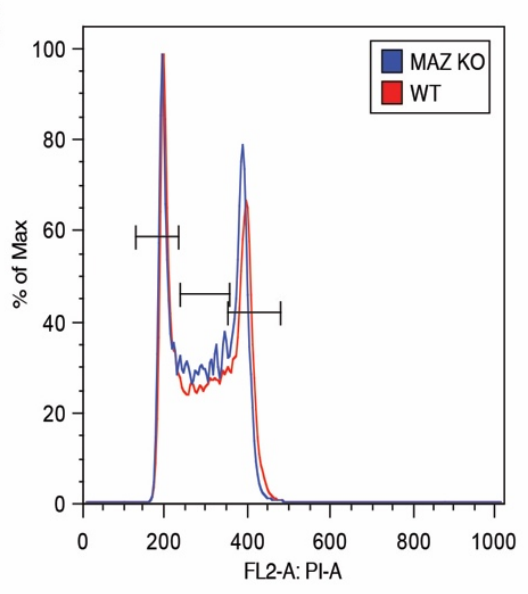

F
B

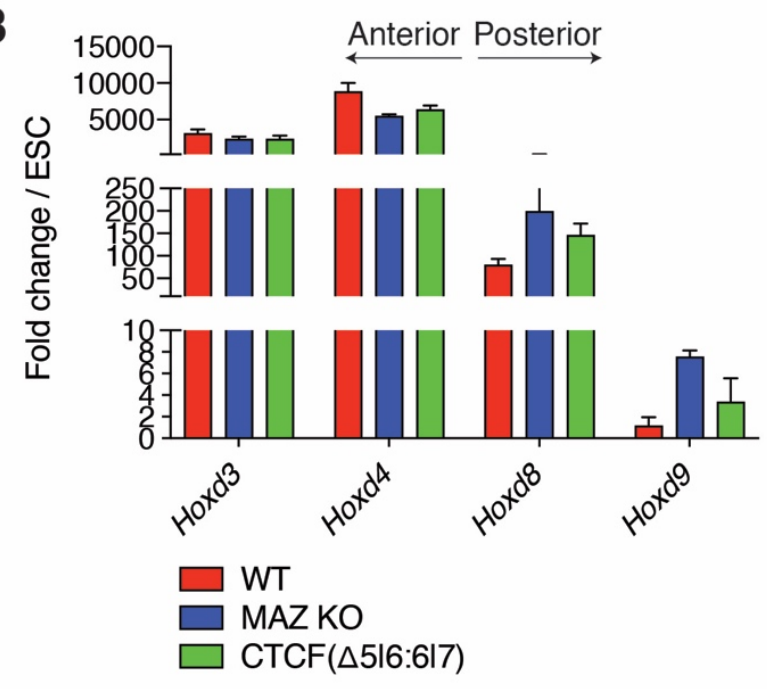

D
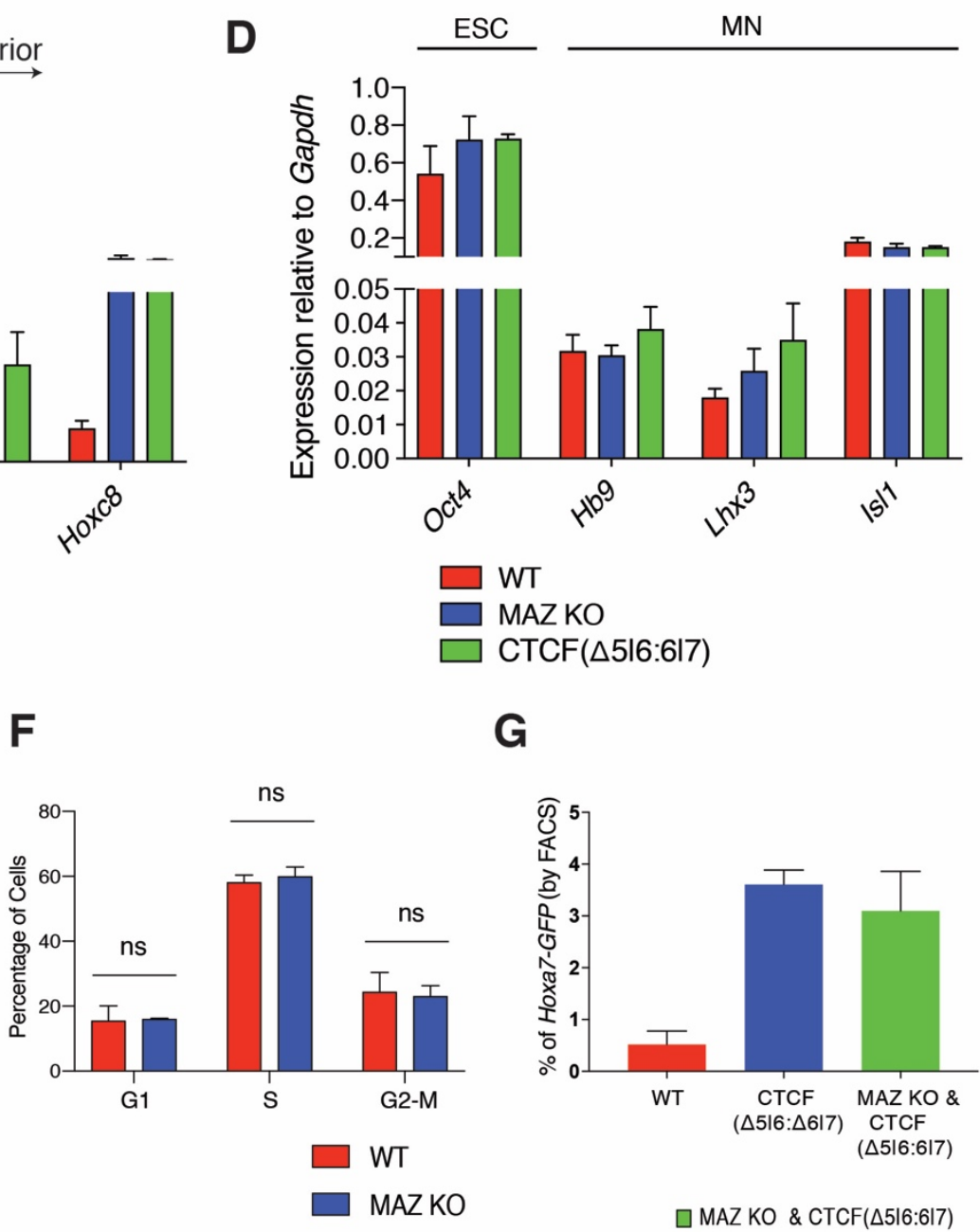

G

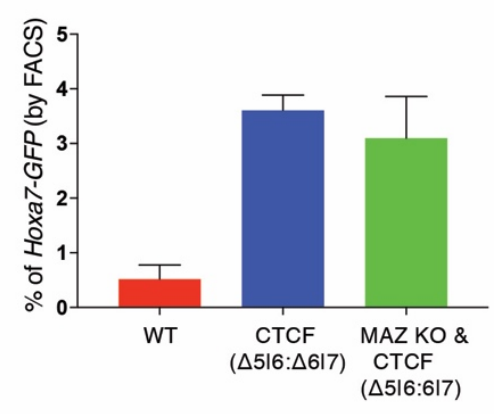

$\square$ MAZ KO \& $\operatorname{CTCF}(\Delta 516: 617)$ 口 $\operatorname{CTCF}(\Delta 566: 617)$ $\square$ WT 
Fig. S6. Loss of Maz results in Hox gene de-repression downstream of CTCF boundaries at Hox A, HoxD, and HoxC clusters

A. RT-qPCR analysis for the indicated Hox genes in HoxA cluster, HoxD cluster (B), and HoxC cluster $(\mathbf{C})$ in WT, MAZ KO, and $\operatorname{CTCF}(\Delta 5|6: 6| 7)$ cells. RT-qPCR signal is normalized to Gapdh levels. Fold change in expression in MNs is calculated relative to baseline expression in ESCs. D. RT-qPCR analysis for the indicated ESC and MN markers in WT, MAZ KO, and CTCF( $\triangle 5|6: 6| 7)$ cells. RT-qPCR signal is normalized to Gapdh levels. Error bars in all RT-qPCR results represent standard deviation across three biological replicates. Maz KO represents three independent clones. E. FACS analysis of cell cycle in WT versus MAZ KO ESCs. F. Quantification of cell cycle analysis by FACS in WT versus MAZ KO ESCs. Error bars indicate standard deviation across three biological replicates. Maz KO represents three independent clones. G. The percentage of Hoxa7-GFP cells quantified based on FACS analysis in MNs with the indicated genotypes: WT, $\operatorname{CTCF}(\Delta 5|6: 6| 7)$, and MAZ KO \& $\operatorname{CTCF}(\Delta 5|6: 6| 7)$. Error bars show standard deviation across three biological replicates. Results from MAZ KO \& $\operatorname{CTCF}(\triangle 5|6: 6| 7)$ represent three independent clones. 
A

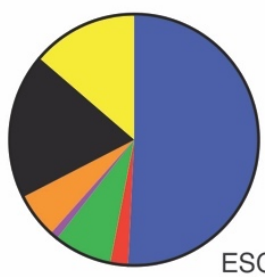

B

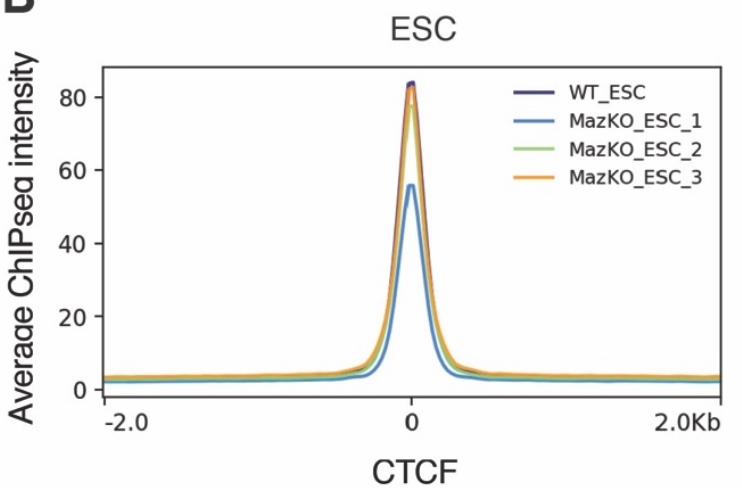

D
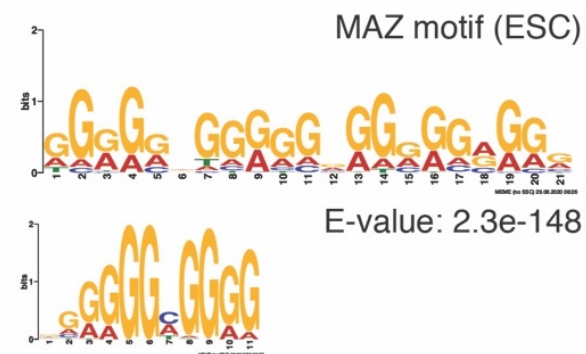

E-value: $5.5 e-010$ peak center

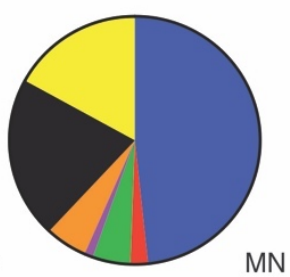

E-value: $2.3 e-148$
$\mathbf{E}$

C

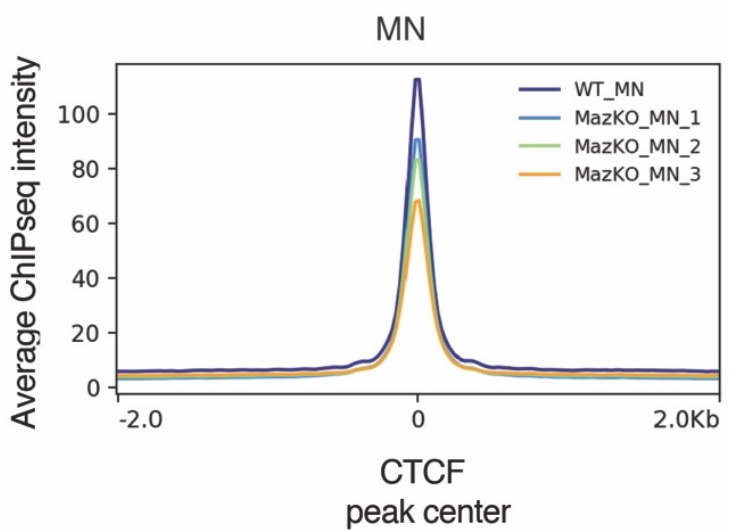

MAZ motif (MN)
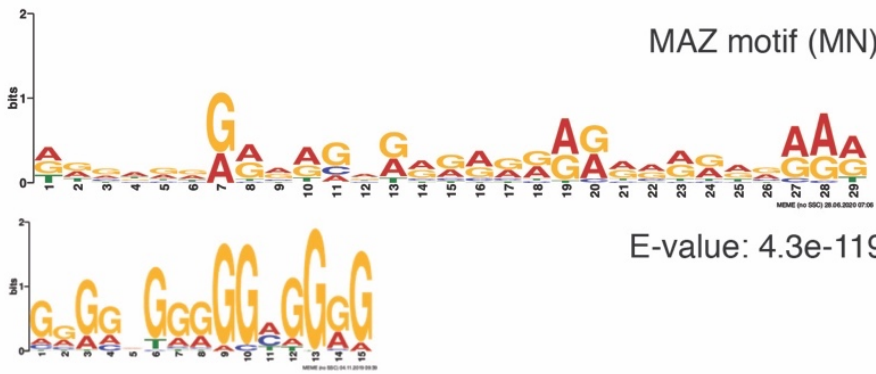

E-value: $4.3 e-119$

F

E-value: 2.3e-031

$10 \mathrm{~kb}$

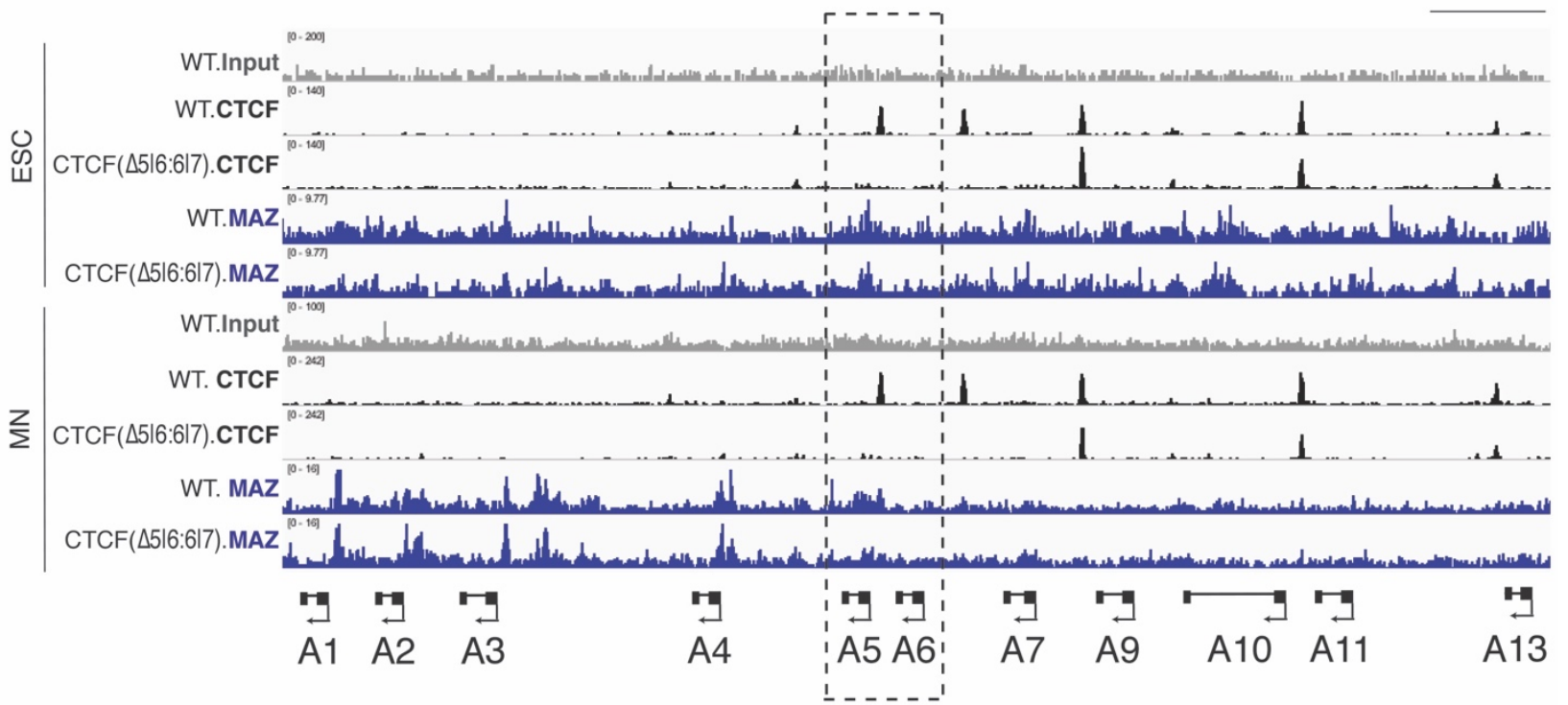




\section{Fig. S7. CTCF binding is altered globally upon MAZ KO in ESCs and MNs}

A. Distribution of MAZ binding sites across genomic features. B. Average CTCF ChIPseq intensity plotted for WT versus MAZ KO ESCs and MNs (C) globally within a 4 kb window centered at CTCF peak center from three biological replicates. D. Motif analysis of MAZ ChIPseq in ESCs, and MNs (E) by using MEME. Top two motifs are shown. F. Normalized ChIP-seq densities for CTCF, and MAZ in WT, and CTCF ( $\Delta 5|6: 6| 7)$ ESCs and MNs in the HoxA cluster. ChIPseq tracks are from one representative of two biological replicates for CTCF and MAZ except for one replicate for MAZ in CTCF $(\Delta 5|6: 6| 7)$ ESCs and MNs. 
A

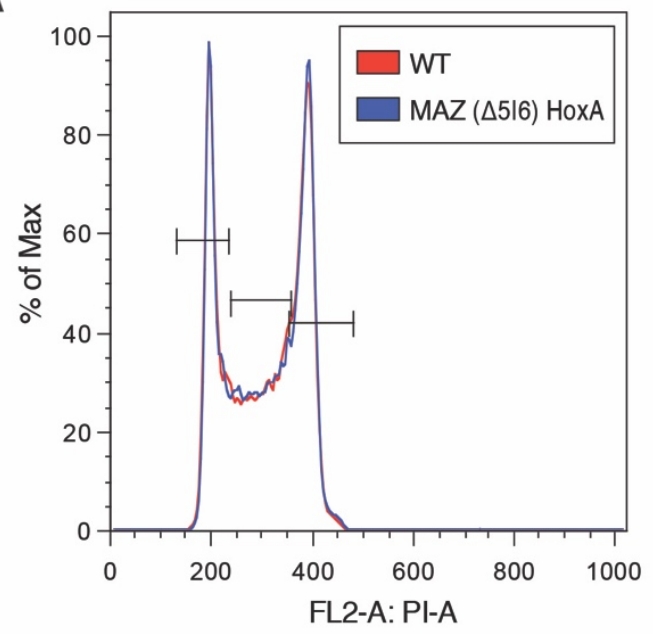

B

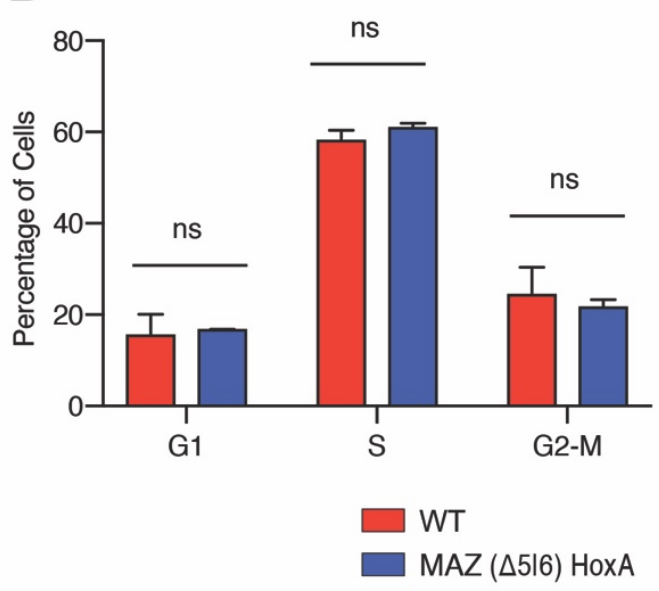

C

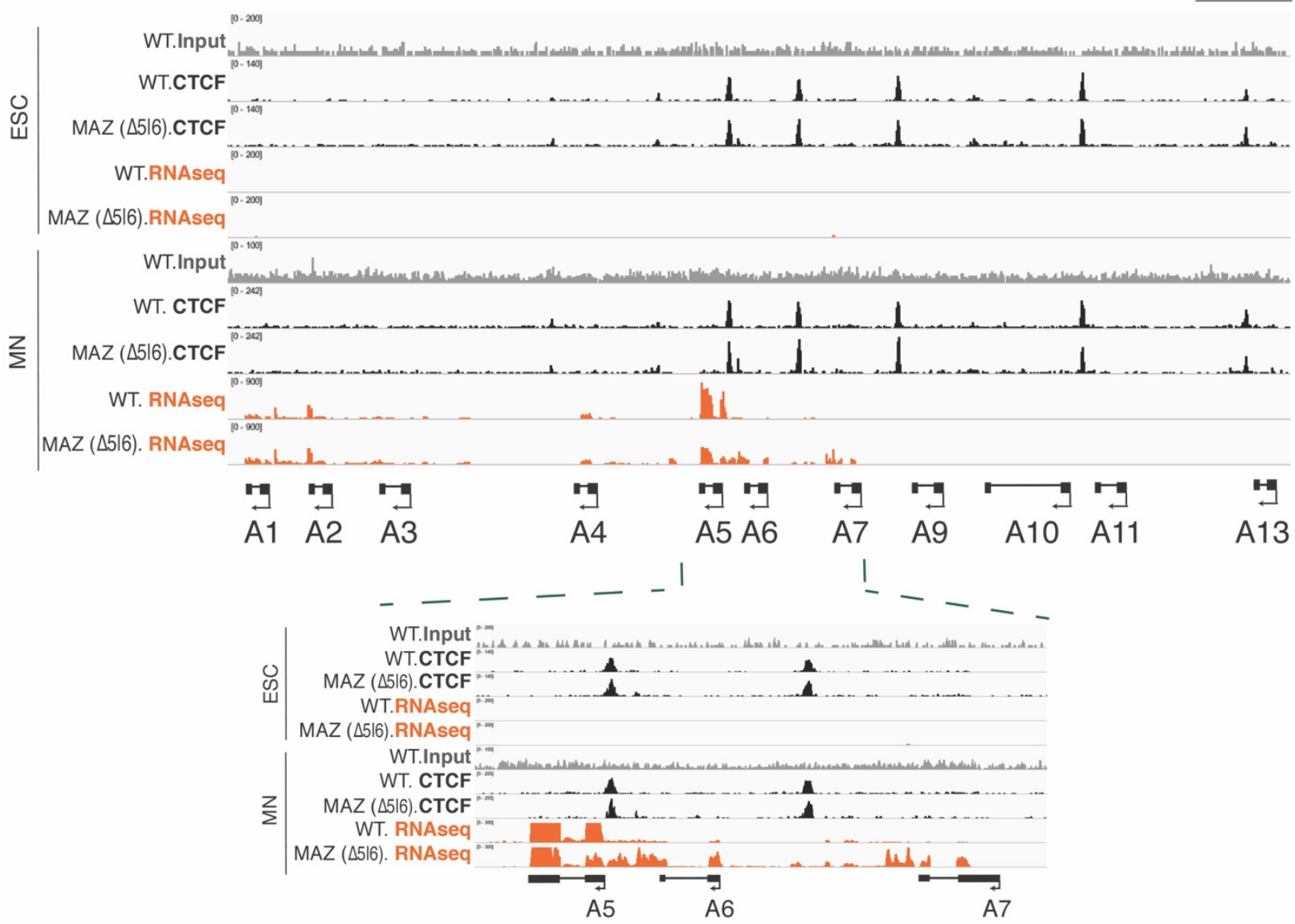


Fig. S8. Loss of MAZ binding site at HoxA cluster alters Hox gene expression pattern at

\section{Hox $A$ cluster}

A. FACS analysis of cell cycle in WT versus MAZ $(\Delta 5 \mid 6)$ ESCs. B. Quantification of cell cycle analysis by FACS in WT versus MAZ $(\Delta 5 \mid 6)$ ESCs. Error bars represent standard deviation across three biological replicates. Student's t-test was used. C. Normalized ChIP-seq densities for CTCF, and RNAseq tracks in WT, and MAZ $(\Delta 5 \mid 6)$ ESCs and MNs in the HoxA cluster. Below is an enlarged view. ChIPseq tracks are from one representative of two biological replicates for CTCF. RNAseq tracks are from one representative of three biological replicates. 

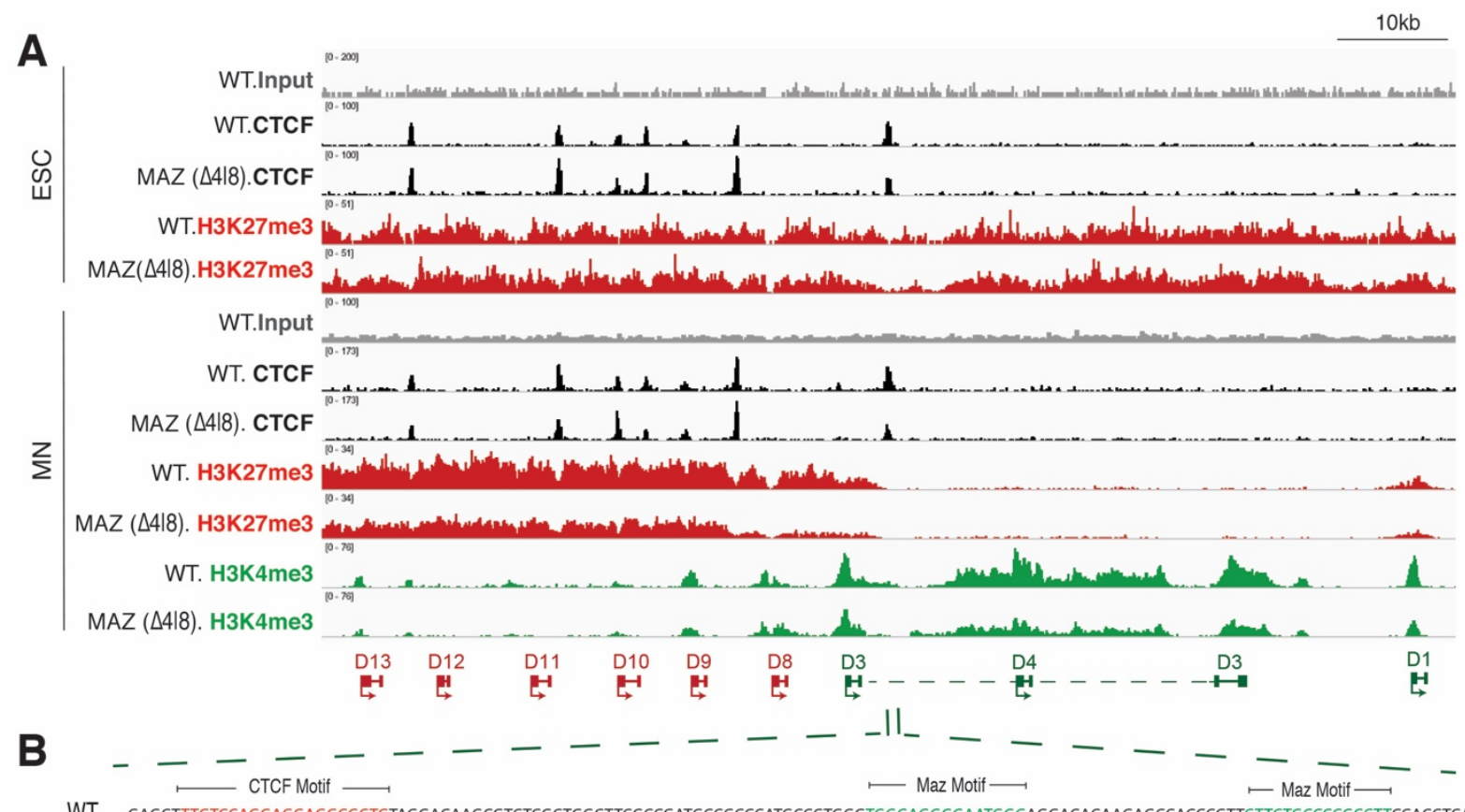

WT GAGCTTTGTCCAGGAGGAGGCGCTGTAGGACAAGCGTCTCGCTGGCTTCGCCGATGCCGCCCATGCCCTGGGTGGGAGGGGAATGGGAGGACACAAGAGCCACCCGTTCTTCTGCCCCGCCTTCCACCTCA

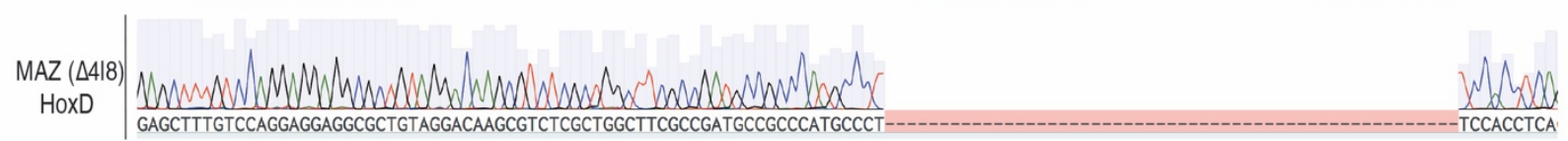

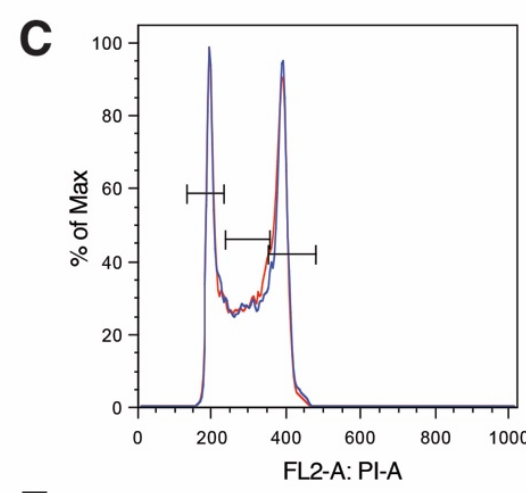

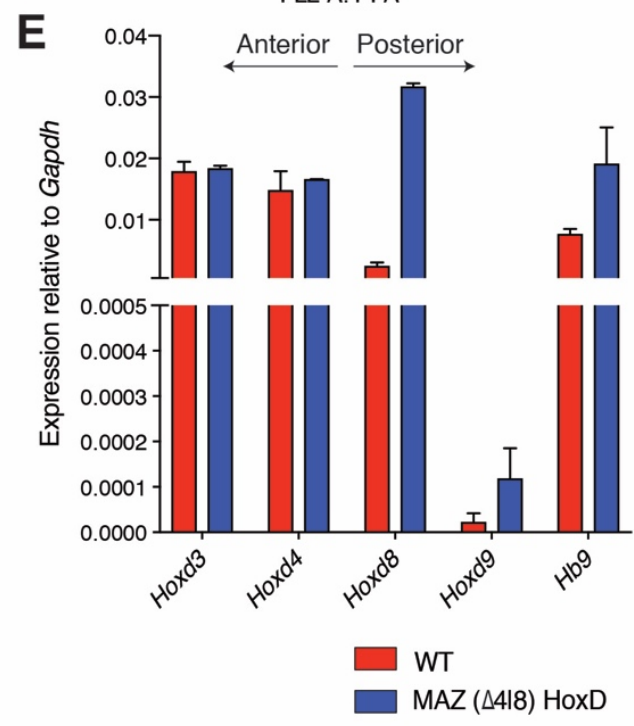

D

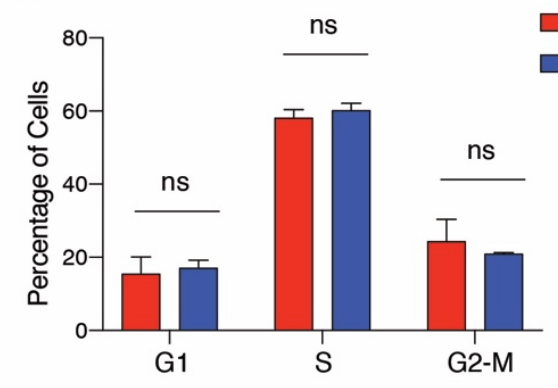

$\mathbf{F}$

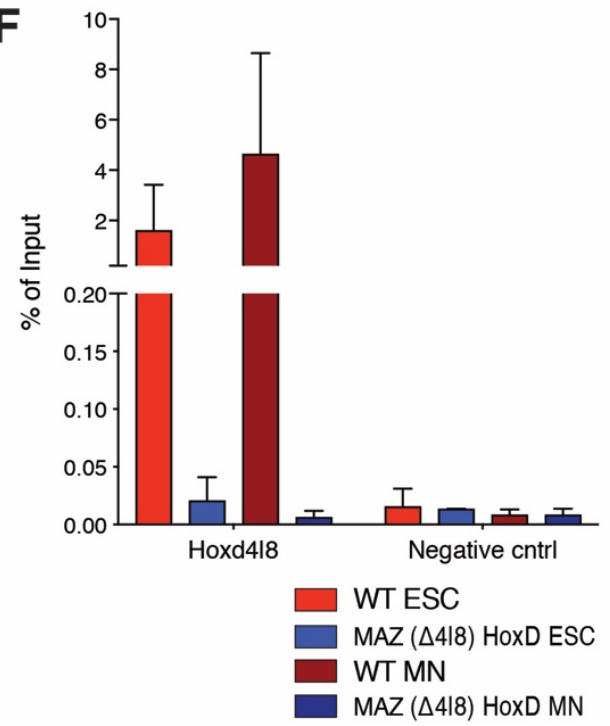


Fig. S9. Loss of MAZ binding site alters Hox gene expression pattern and chromatin domains

\section{at $H o x D$ cluster}

A. Normalized ChIP-seq densities for CTCF and indicated histone post-translational modifications (PTMs) in WT, and MAZ $(\Delta 4 \mid 8)$ ESCs and MNs in the HoxD cluster. ChIPseq tracks are from one representative of two biological replicates for CTCF, and one replicate for histone PTMs. B. MAZ binding site deletion via CRISPR is depicted for the $4 \mid 8$ site at the HoxD cluster. C. FACS analysis of cell cycle in WT versus MAZ $(\Delta 4 \mid 8)$ ESCs. D. Quantification of cell cycle analysis by FACS in WT versus MAZ $(\Delta 4 \mid 8)$ ESCs. Error bars represent standard deviation across three biological replicates. Student's t-test was used. E. RT-qPCR for the indicated Hox genes in the HoxD cluster in MNs upon MAZ $(\Delta 4 \mid 8)$. Error bars indicate standard deviation across two biological replicates. F. MAZ ChIP-qPCR analysis in the HoxD cluster in mESCs and MNs upon MAZ $(\Delta 4 \mid 8)$. Error bars show standard deviation across two biological replicates. 
A

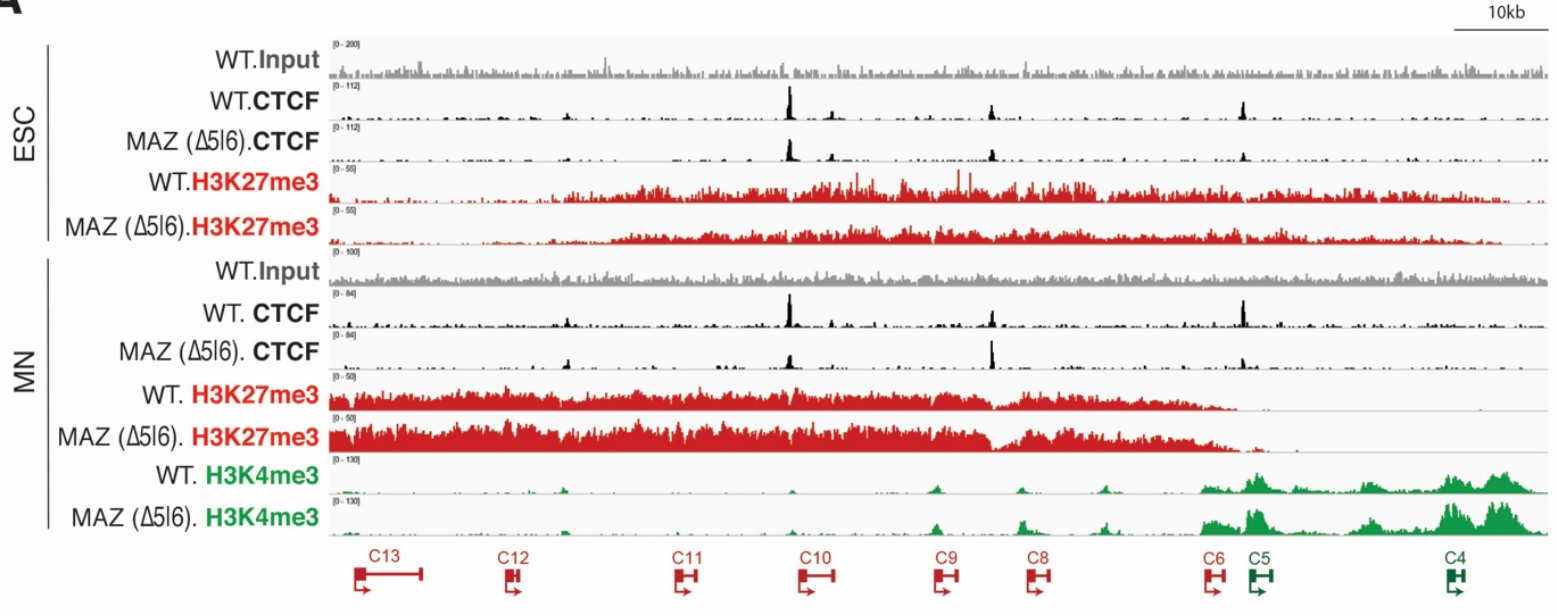

B

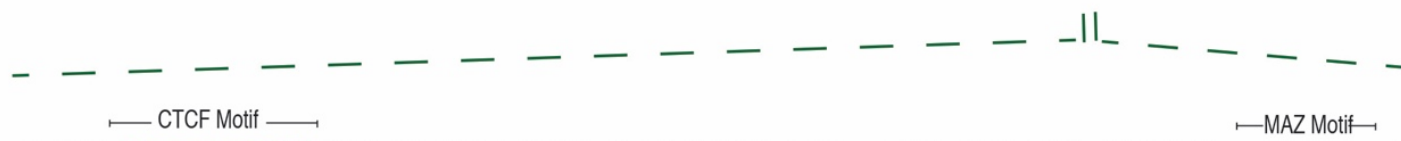

WT TCCTCCGAGTCGGCACCAGGTGTCGCTGTGAGCTCGTTGTCCCGACTACCCCCCAATTCCAAGAACCAttHtHttCCCTCCCCCTTGCTTCTCTTTCTCTTTCATCCCCCCCCCCCCaacCCCCG

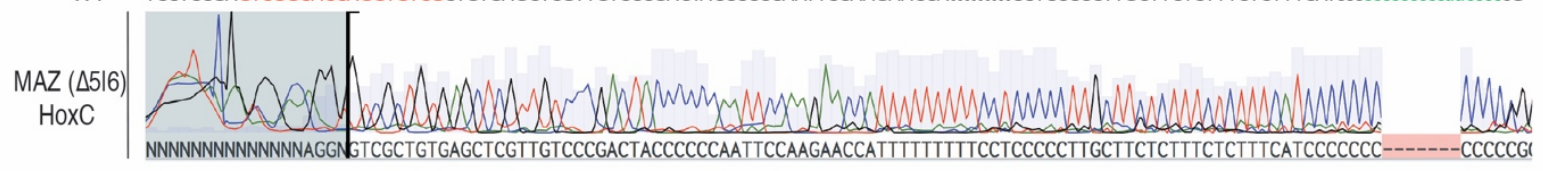

C

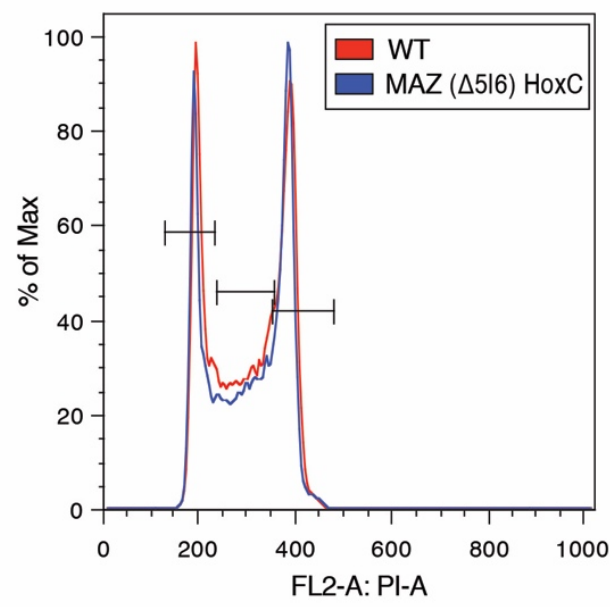

D

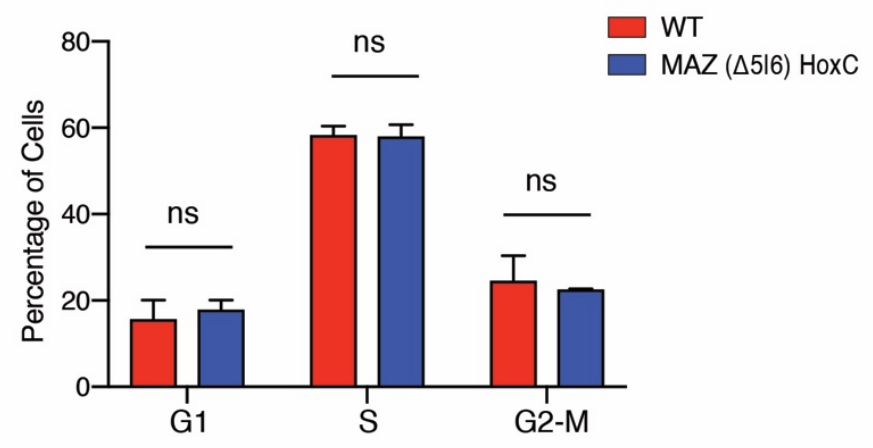

E

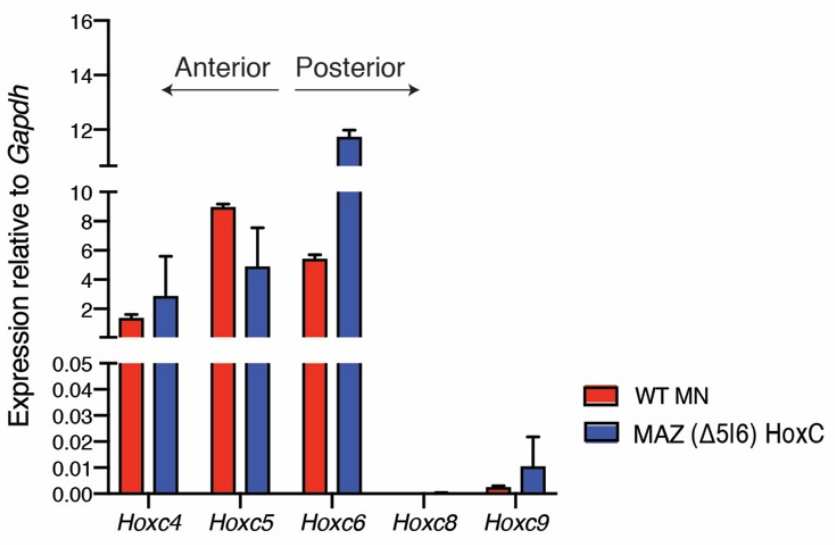


Fig. S10. Loss of MAZ binding site alters Hox gene expression pattern and chromatin domains at the HoxC cluster

A. Normalized ChIP-seq densities for CTCF, and indicated histone PTMs in WT, and MAZ ( $\Delta 5 \mid 6)$ ESCs and MNs in the HoxC cluster. ChIPseq tracks represent one biological replicate. B. MAZ binding site deletion via CRISPR is depicted for the $5 \mid 6$ site at the HoxC cluster. C. FACS analysis of cell cycle in WT versus MAZ $(\Delta 5 \mid 6)$ ESCs. D. Quantification of cell cycle analysis by FACS in WT versus MAZ $(\Delta 5 \mid 6)$ ESCs. Error bars represent standard deviation across three biological replicates. Student's t-test was used. E. RT-qPCR for the indicated Hox genes in the HoxC cluster in MNs upon MAZ ( $\Delta 5 \mid 6)$. Error bars show standard deviation across two biological replicates. 
A

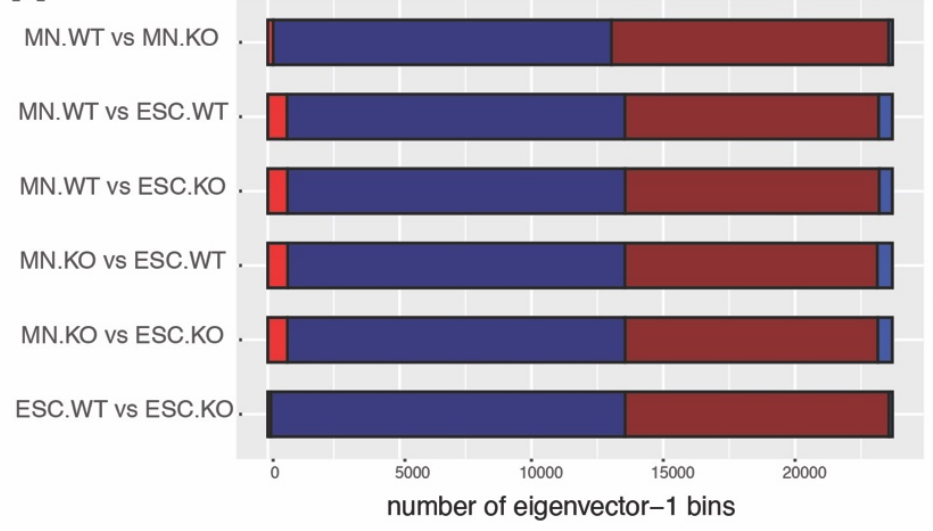

C
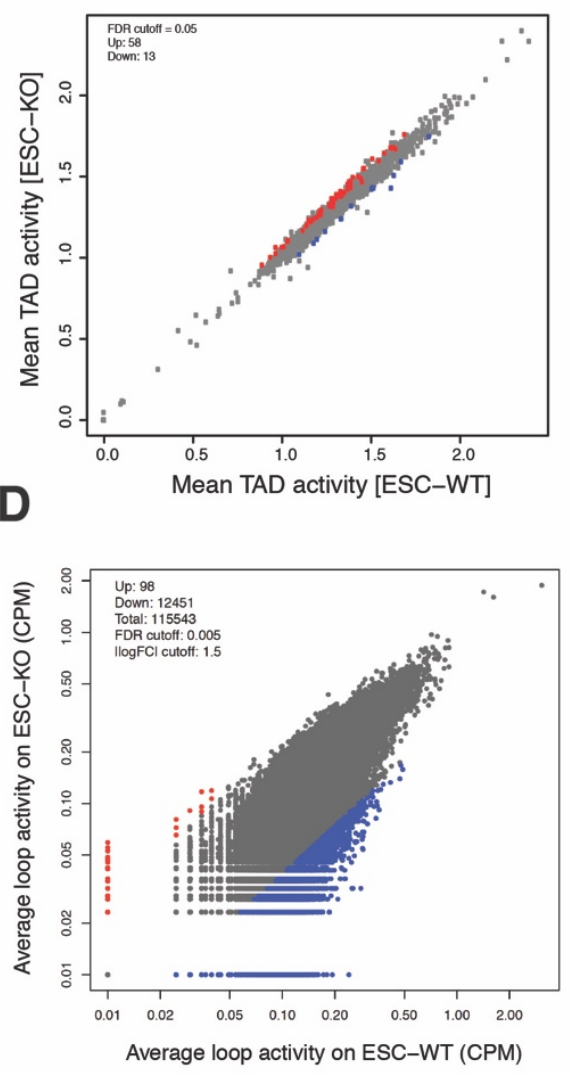

E
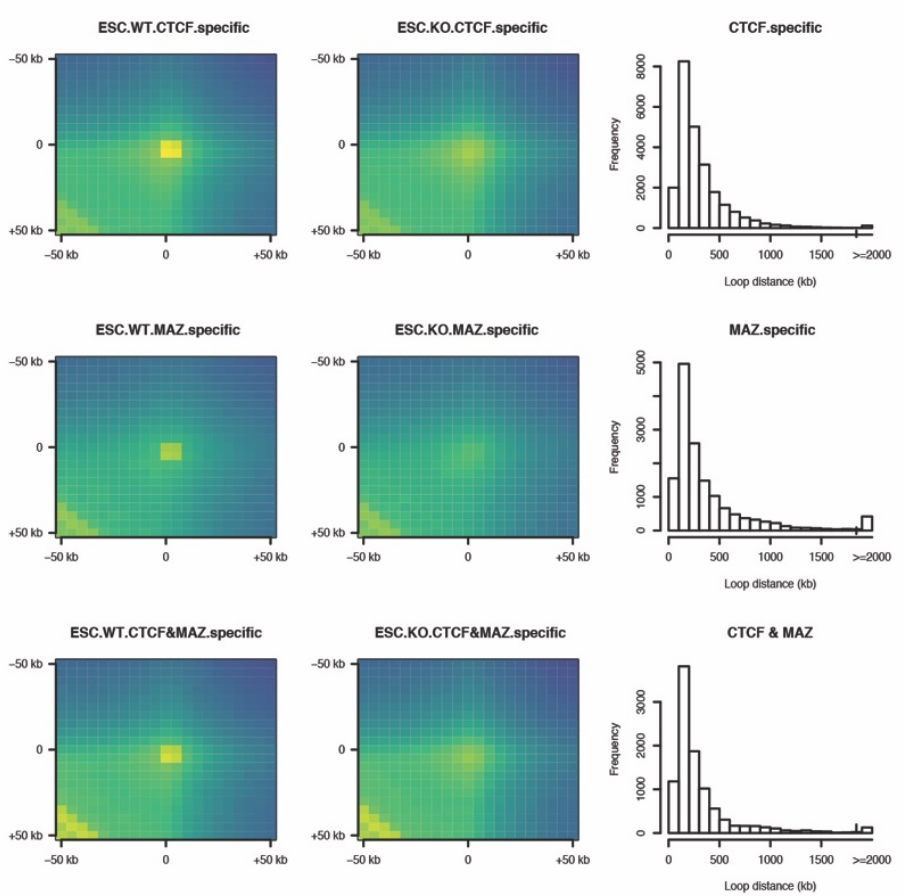

\section{Fig. S11. HiC analysis of global organization in MAZ KO ESCs and MNs}

A. Bar plot of compartment switches between active (A) and inactive (B) compartments in ESCs and MNs. B. Principal Component Analysis (PCA) of boundary scores in WT ESCs, MAZ KO ESCs, WT MNs, and MAZ KO MNs. C. Scatter plot showing differential intra-TAD activity in 
WT vs MAZ KO ESCs (FDR cut-off=0.05). D. Scatter plot showing differential loop activity in WT vs MAZ KO ESCs (all loops, n=115543, FDR cut-off=0.005, $\mid \log$ (Fold Change) $\mid$ cut-off=1.5, Up-regulated=98, Down-regulated: 12451). E. Aggregate Peak Analysis (APA) of loops in WT vs MAZ KO ESCs showing ChIP-seq signals of CTCF, MAZ, or both at any region covered by them. The resolution of APA is $5 \mathrm{~kb}$. Histograms showing the distribution of loop distance in MAZ KO compared to WT related to the binding level of ChIP-seq. 

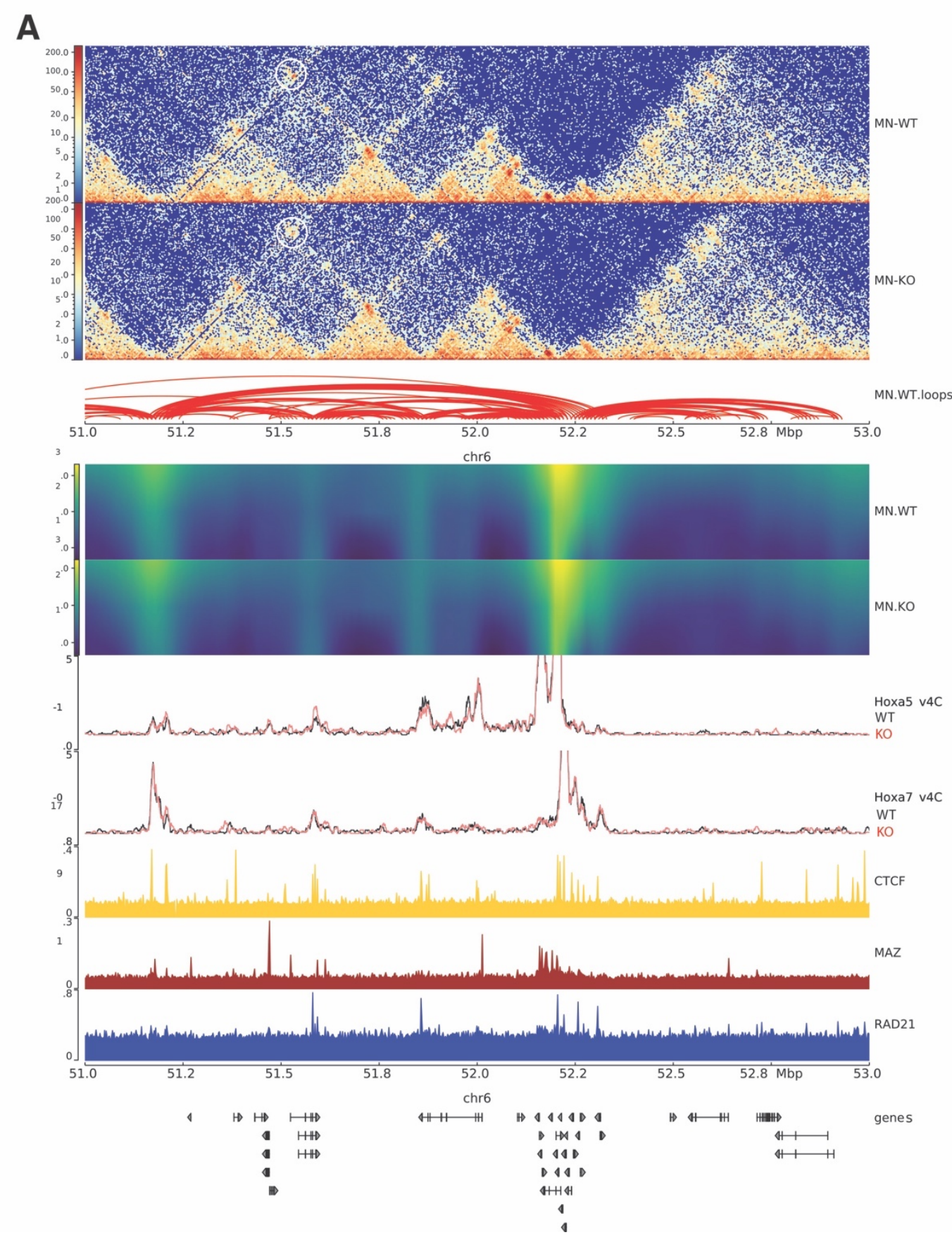

Fig. S12. Visualization of HiC analysis at the indicated region in WT and MAZ KO MNs

A. Visualization of Hi-C contact matrices for a zoomed-in region on chromosome 6 with a change of loop activity in WT vs MAZ KO MNs. Shown below are loops identified in WT MNs, insulation score heat maps in WT MNs and MAZ KO MNs, virtual 4C plots with Hoxa5 as viewpoint and 
bioRxiv preprint doi: https://doi.org/10.1101/2020.08.25.267237; this version posted August 26, 2020. The copyright holder for this preprint (which was not certified by peer review) is the author/funder. All rights reserved. No reuse allowed without permission.

Hoxa7 as viewpoint, and ChIPseq read densities for CTCF, MAZ, and RAD21. The last track shows gene annotations. 
bioRxiv preprint doi: https://doi.org/10.1101/2020.08.25.267237; this version posted August 26, 2020. The copyright holder for this preprint (which was not certified by peer review) is the author/funder. All rights reserved. No reuse allowed without permission.
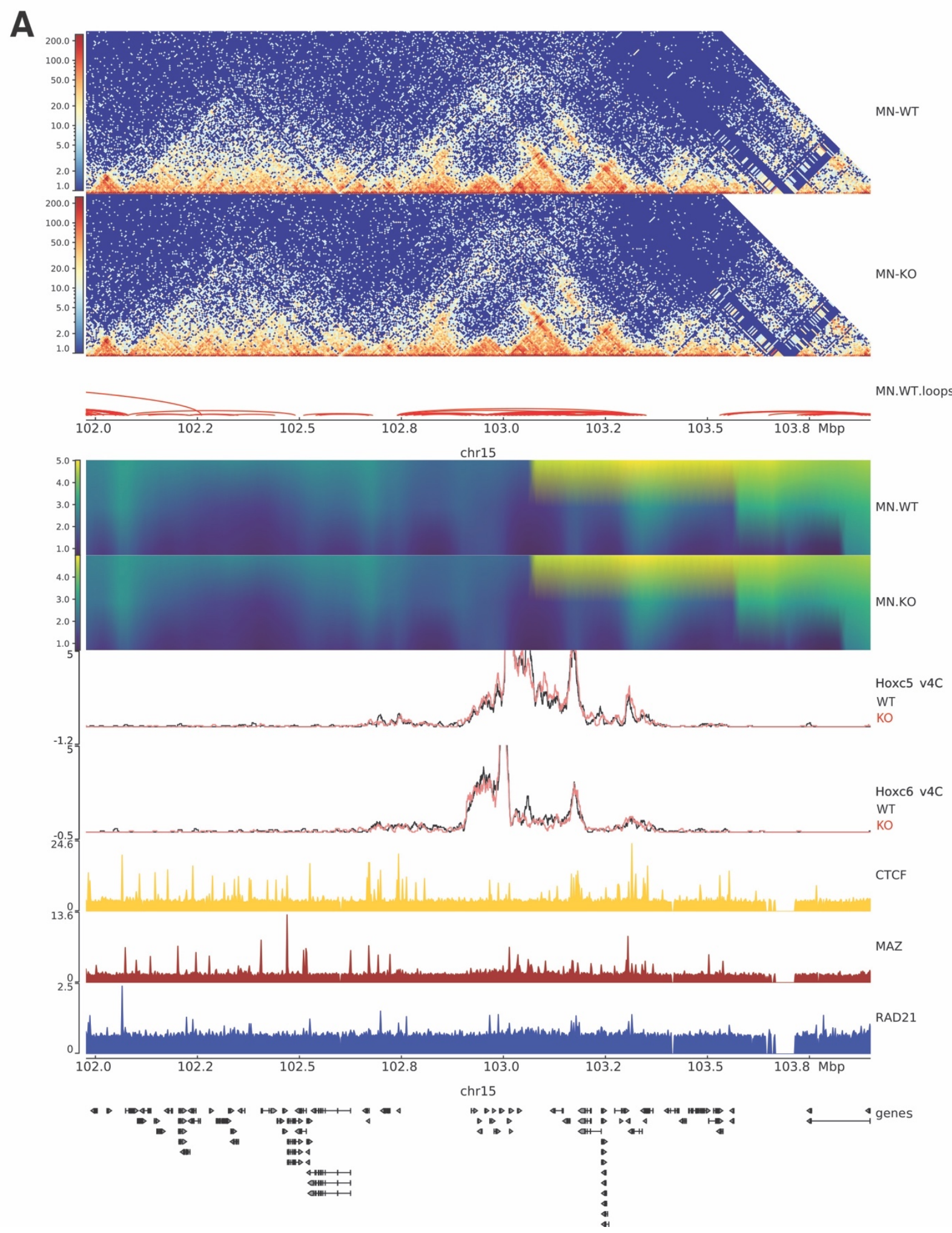
Fig. S13. Visualization of $\mathrm{HiC}$ analysis at the indicated region in WT and MAZ KO MNs

A. Visualization of Hi-C contact matrices for a zoomed-in region on chromosome 15 in WT vs MAZ KO MNs. Shown below are loops identified in WT MNs, insulation score heat maps in WT MNs and MAZ KO MNs, virtual 4C plots with Hoxc5 as viewpoint and Hoxc6 as viewpoint, and ChIPseq read densities for CTCF, MAZ, and RAD21. The last track shows gene annotations. 


\section{A}
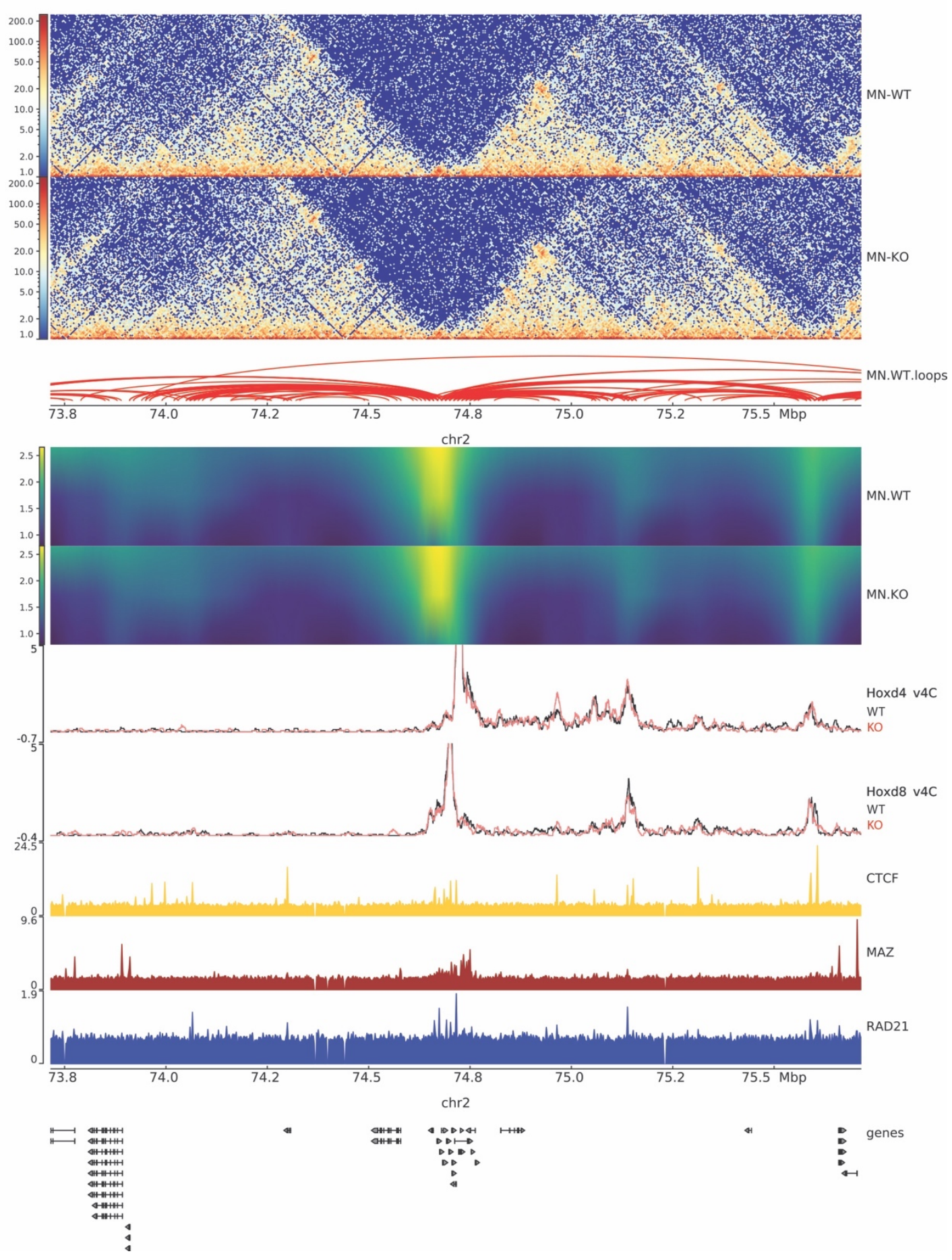

Fig. S14. Visualization of $\mathrm{HiC}$ analysis at the indicated region in WT and MAZ KO MNs

A. Visualization of Hi-C contact matrices for a zoomed-in region on chromosome 2 in WT vs MAZ KO MNs. Shown below are loops identified in WT MNs, insulation score heat maps in WT 
bioRxiv preprint doi: https://doi.org/10.1101/2020.08.25.267237; this version posted August 26, 2020. The copyright holder for this preprint (which was not certified by peer review) is the author/funder. All rights reserved. No reuse allowed without permission.

MNs and MAZ KO MNs, virtual 4C plots with Hoxd4 as viewpoint and Hoxd8 as viewpoint, and ChIPseq read densities for CTCF, MAZ, and RAD21. The last track shows gene annotations. 
bioRxiv preprint doi: https://doi.org/10.1101/2020.08.25.267237; this version posted August 26, 2020. The copyright holder for this preprint

(which was not certified by peer review) is the author/funder. All rights reserved. No reuse allowed without permission.

\section{TABLE S1}

CRISPR sgRNAs, DONORS, GENOTYPING PRIMERS

\begin{tabular}{|c|c|c|c|c|}
\hline Primer Name & Forward Sequence & Reverse Sequence & sgRNA target & Aim \\
\hline A5.Cterm.gRNA & CACCGTTTGGCCGCTCAGATGCTC & AAACGAGCATCTGAGCGGCCAAAC & Hoxa5 & Hoxa5-P2A-mCherry knock-in \\
\hline A7.Cterm.gRNA & CACCGGCTGACAAGGCGGACGAGG & AAACCCTCGTCCGCCTTGTCAGCC & Hoxa7 & Hoxa7-P2A-eGFP knock-in \\
\hline HOX5|6 gRNA.3 & CACCGGGACTCGAGCGCCACCCGC & AAACGCGGGTGGCGCTCGAGTCCC & CTCF binding site at Hoxa $5 \mid 6$ & CTCF binding site deletion \\
\hline Hox6|7.gRNA.new & CACCGGCTCGCTGCTGCCACGCTG & AAACCAGCGTGGCAGCAGCGAGCC & CTCF binding site at Hoxa6 $\mid 7$ & CTCF binding site deletion \\
\hline CTCF.Cterm.gRNA & CACCGCATGATGGACCGGTGATGC & AAACGCATCACCGGTCCATCATGC & Ctcf & CTCF C-terminus FLAG tag knock-in \\
\hline Maz ex2 2 & CACCGCCGAATGCGACAGCTTATGT & AAACACATAAGCTGTCGCATTCGGC & Maz & MAZ knock-out \\
\hline Maz.a56.gRNA1 & CACCGGAGTTGGGTGGAGGCAAGA & AAACTCTTGCCTCCACCCAACTCC & MAZ binding site at Hoxa $5 \mid 6$ & MAZ binding site deletion \\
\hline Maz.d48.gRNA2*** & CACCGGATGCCGCCCATGCCCTGGG & AAACCCCAGGGCATGGGCGGCATCC & MAZ binding site at Hoxd $4 \mid 8$ & MAZ binding site deletion \\
\hline Maz.d48.gRNA3** & CACCGGCGAGCTGAGGTGGAAGGCG & AAACCGCCTTCCACCTCAGCTCGCC & MAZ binding site at $\operatorname{Hoxd} 4 \mid 8$ & MAZ binding site deletion \\
\hline Maz.c56.gRNA1 & CACCGGGCAAAGCCCGGGGGTTGGG & AAACCCCAACCCCCGGGCTTTGCCC & MAZ binding site at $H o x c 5 \mid 6$ & MAZ binding site deletion \\
\hline **simultaneously trans & $\begin{array}{l}\text { *G for U6 promoter transcription initiation } \\
\text { *BbsI overhang }\end{array}$ & *BbsI overhang & & \\
\hline
\end{tabular}

\begin{tabular}{|c|c|c|c|}
\hline \multicolumn{4}{|l|}{ Genotyping Primers } \\
\hline Experiment & Primer Name & Forward Sequence & Reverse Sequence \\
\hline Hoxa $5: a 7$ dual reporter genotyping & A5.AH1.int.F/A5.AH2.int.R & GACAATATAGGTGGCCCAGAAG & GATGAATTAGGGCAACGAGAAC \\
\hline Hoxa5:a7 dual reporter genotyping & A7.AH1.int.F/A7.AH2.int.R & TCCACATCCTAGTCTGCTTGTC & TCTTAAAGACGCTTTTCCAACTG \\
\hline Hoxa5:a 7 dual reporter genotyping & A5.AH1.int.F/mCherry.begin.R & GACAATATAGGTGGCCCAGAAG & GTACATGAACTGAGGGGACAGG \\
\hline Hoxa $5: a 7$ dual reporter genotyping & A7.AH1.int.F/EGFP.begin.R & TCCACATCCTAGTCTGCTTGTC & GCAGATGAACTTCAGGGTCAG \\
\hline Hoxa5:a 7 dual reporter genotyping & mCherry.end.F/A5.out.R & CAACATCAAGTTGGACATCACC & CTCAATTCAGTCTTGCCAAATG \\
\hline Hoxa5:a 7 dual reporter genotyping & EGFP.end.F/A7.out.R & ACATGGTCCTGCTGGAGTTC & CCAGAGGACGCAGGAAATTAG \\
\hline Hoxa5:a 7 dual reporter genotyping & A5.out.F/P2A.R & TGGTACATCCTAATGGAACTGC & TCAGCAGAGAGAAGTTTGTTGC \\
\hline Hoxa5:a 7 dual reporter genotyping & A7.out.F/P2A.R & AATGGGGTTTGGTGTAAATCTG & TCAGCAGAGAGAAGTTTGTTGC \\
\hline Hoxa5:a 7 dual reporter genotyping & A5.out.F/mCherry.begin. $R$ & TGGTACATCCTAATGGAACTGC & GTACATGAACTGAGGGGACAGG \\
\hline Hoxa5:a 7 dual reporter genotyping & A7.out.F/EGFP.begin.R & AATGGGGTTTGGTGTAAATCTG & GCAGATGAACTTCAGGGTCAG \\
\hline Hoxa5:a 7 dual reporter genotyping & pBluescript.out.F1/mCherry.begin.R & CAAGGCGATTAAGTTGGGTAAC & GTACATGAACTGAGGGGACAGG \\
\hline Hoxa $5: a 7$ dual reporter genotyping & pBluescript.out.F1/EGFP.begin.R & CAAGGCGATTAAGTTGGGTAAC & GCAGATGAACTTCAGGGTCAG \\
\hline FLAG-CTCF knock-in cell line genotyping & CTCF.CFtag.F1/R1 & AAAAAGGAGCCAGATGCCGA & GCCGTTTAAACACAGCCCAA \\
\hline CTCF binding site deletion $($ Hoxa $5 \mid 6)$ & 5|6.494.new2.f/r & CACCCTTGCACAATTTATGATGA & GGATACAAAGCCGGGGAAATAA \\
\hline CTCF binding site deletion (Ноха6 $\mid 7)$ & HOX6|7.PCR.530.f/r & TGTACAAACAGTCTCCATGGTG & GTTCCCTGGCTATGGTTCTTTT \\
\hline MAZ knock-out & Maz_ex2_g2 F1/R1 & AAGCGCATCCGGAAGAATCA & CAGTGGGAGCAGTTGTAGGG \\
\hline MAZ knock-out & Maz ex2 g2 F2/R2 & TCGGGTGCTATGAAGATGCC & CAGTGGGAGCAGTTGTAGGG \\
\hline MAZ binding site deletion $($ Hoxa $5 \mid 6)$ & Maz.a56.geno.F1/R1 & TGACTGGGACATGTACTCGG & TGGGCTGTAACCTCAATTCGA \\
\hline MAZ binding site deletion $($ Hoxa $5 \mid 6)$ & Maz.a56.geno.F2/R2 & TCGGTTCCCTCCTACGTAGG & GCTCGAGTCCGACTGAACG \\
\hline MAZ binding site deletion (Hoxd4 $\mid 8$ ) & Maz.d48.geno.F1/R1 & TTTCGGTTGTCTGGAGCTTT & CGGACAAGTGATCACACCAC \\
\hline MAZ binding site deletion $(\operatorname{Hoxd} 4 \mid 8)$ & Maz.d48.geno.F2/R2 & GGACTCCTTTTTGCCTCTCC & CGGACAAGTGATCACACCAC \\
\hline MAZ binding site deletion $(\operatorname{Hoxc} 5 \mid 6)$ & Maz.c56.geno.F1/R1 & GCCAAGCGATGCTACAAGAT & TGAAGTTGAGGGGGTTTTTG \\
\hline
\end{tabular}

\begin{tabular}{|c|c|}
\hline \multicolumn{2}{|l|}{ Oligos/Donor Plasmid } \\
\hline \multicolumn{2}{|c|}{ Hoxa5-P2A-mCherry knock-in (pBlueScript SK+ containing AH1-P2A-mCherry-AH2) } \\
\hline $\begin{array}{l}\text { A5 arm of homology } 1 \\
\text { (AH1) }\end{array}$ & 2004 bp \\
\hline P2A.mCherry.cassette & $\begin{array}{l}\text { GGATCCGGCGCAACAAACTTCTCTCTGCTGAAACAAGCCGGAGATGTCGAAGAGAATCCCGGTCCTATGGTGAGCAAGGGCGAGGAGGATAAC } \\
\text { ATGGCCATCATCAAGGAGTTCATGCGCTTCAAGGTGCACATGGAGGGCTCCGTGAACGGCCACGAGTTCGAGATCGAGGGCGAGGGCGAGGGC } \\
\text { CGCCCCTACGAGGGCACCCAGACCGCCAAGCTGAAGGTGACCAAGGGTGGCCCCCTGCCCTTCGCCTGGGACATCCTGTCCCTCAGTTCATGT } \\
\text { ACGGCTCCAAGGCTACGTGAAGCACCCGCCGACATCCCCACTACTTGAAGCTGTCCTTCCCCGAGGGCTTCAAGTGGGAGCGCGTGATGAA } \\
\text { CTTCGAGGACGGCGGCGTGGTGACCGTGACCCAGGACTCCTCCCGCAGGACGGCGAGTCATCTACAAGGTGAAGCTGCGCGGCACCAACTTC } \\
\text { CCCTCCGACGGCCCCGTAATGCAGAAGAAGACCATGGGCTGGGAGGCCTCCTCCGAGCGGATGTACCCCGAGGACGGCGCCCTGAAGGGCGAG } \\
\text { ATCAAGCAGAGGCTGAAGCTGAAGGACGGCGGCCACTACGACGCTGAGGTCAAGACCACCTACAAGGCCAAGAAGCCCGTGCAGCTGCCCGGC } \\
\text { GCCTACAACGTCAACATCAAGTTGGACATCACCTCCCACAACGAGGACTACACCATCGTGGAACAGTACGAACGCGCCGAGGGCCGCCACTCC } \\
\text { ACCGGCGGCATGGACGAGCTGTACAAGGAATTCTAA }\end{array}$ \\
\hline $\begin{array}{l}\text { A5 arm of homology } 2 \\
(\mathrm{AH} 2)\end{array}$ & $2225 \mathrm{bp}$ \\
\hline \multicolumn{2}{|c|}{ Hoxa7-P2A-eGFP knock-in (pBlueScript SK+ containing AH1-P2A-eGFP-AH2) } \\
\hline $\begin{array}{l}\text { A7 arm of homology } 1 \\
\text { (AH1) }\end{array}$ & $1698 \mathrm{bp}$ \\
\hline P2A.eGFP.cassette & 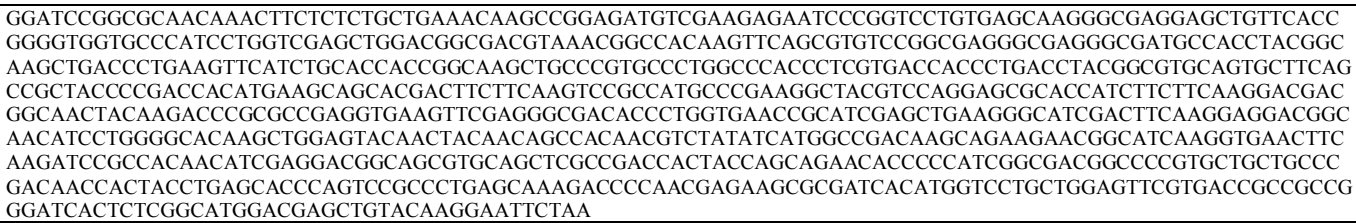 \\
\hline $\begin{array}{l}\text { A7 arm of homology } 2 \\
\text { (AH2) }\end{array}$ & $2323 \mathrm{bp}$ \\
\hline \multicolumn{2}{|c|}{ CTCF C-terminus Flag tag knock-in (ssDNA oligo) } \\
\hline CTCF.Cftag.oligo & $\begin{array}{l}\text { GCTCAGGCAGCCACCACAGACGCCCCCAACGGAGACCTCACGCCTGAGATGATCCTCAGCATGATGGACCGGGGATCCGCTGACTACAAGGAT } \\
\text { GACGACGATAAGTGATGCTGGGGCCTTGCTCGGCACCAGGACTATTGGGCTGTGTTTAAACGGCCCAAATCTTAATTTTTCTCTTTTTTTTCTTTG } \\
\text { GCTTTGGGAAC }\end{array}$ \\
\hline
\end{tabular}

Table S1. List of CRISPR sgRNAs, donors, and genotyping primers 
bioRxiv preprint doi: https://doi.org/10.1101/2020.08.25.267237; this version posted August 26, 2020. The copyright holder for this preprint (which was not certified by peer review) is the author/funder. All rights reserved. No reuse allowed without permission.

\section{TABLE S2}

\section{OLIGOS, ANTIBODIES}

\begin{tabular}{|c|c|c|}
\hline \multicolumn{3}{|c|}{ RT-qPCR Primers } \\
\hline Primer Name & Forward Sequence & Reverse Sequence \\
\hline Hoxa1 & ACCAAGAAGCCTGTCGTTCC & TAGCCGTACTCTCCAACTTTCC \\
\hline Hoxa2 & CCTGGATGAAGGAGAAGAAGG & GTTGGTGTACGCGGTTCTCA \\
\hline Hoxa3 & TCAAGGCAGAACACTAAGCAGA & ATAGGTAGCGGTTGAAGTGGAA \\
\hline Hoxa4 & TGTACCCCTGGATGAAGAAGAT & AAGACTTGCTGCCGGGTATAG \\
\hline Hoxa5 & TGTACGTGGAAGTGTTCCTGTC & GTCACAGTTTTCGTCACAGAGC \\
\hline Hoxa6 & ACCGACCGGAAGTACACAAG & AGGTAGCGGTTGAAGTGGAAT \\
\hline Hoxa7 & GAAGCCAGTTTCCGCATCTAC & CTTCTCCAGTTCCAGCGTCT \\
\hline Hoxa9 & TCCCTGACTGACTATGCTTGTG & ATCGCTTCTTCCGAGTGGAG \\
\hline Hoxa10 & GAAGAAACGCTGCCCTTACAC & TTTCACTTGTCTGTCCGTGAG \\
\hline Hoxa11 & CGAGAGTTCTTCTTCAGCGTCT & TGGAGCCTTAGAGAAGTGGATT \\
\hline Hoxa13 & GCGGTGTCCATGTACTTGTC & GCTGCCCTACGGCTACTTC \\
\hline Hoxd3 & CTACCCTTGGATGAAGAAGGTG & TCAGACAGACACAGGGTGTGA \\
\hline Hoxd4 & CTACCCTTGGATGAAGAAGGTG & TTCTAGGACTTGCTGTCTGGTG \\
\hline Hoxd8 & GCTCGTCTCCTTCTCAAATGTT & GCGACTGTAGGTTTGTCTTCCT \\
\hline Hoxd 9 & CAGCAACTTGACCCAAACAAC & TGGTATTTGGTGTAGGGACAGC \\
\hline Hoxc4 & AGCAAGCAACCCATAGTCTACC & GCGGTTGTAATGAAACTCTTTCTC \\
\hline Hoxc5 & CACAGATTTACCCGTGGATGAC & CTTTCTCGAGTTCCAGGGTCT \\
\hline Hoxc6 & TAGTTCTGAGCAGGGCAGGA & CGAGTTAGGTAGCGGTTGAAGT \\
\hline Hoxc8 & GTAAATCCTCCGCCAACACTAA & CGCTTTCTGGTCAAATAAGGAT \\
\hline Hoxc9 & GCAAGCACAAAGAGGAGAAGG & CGTCTGGTACTTGGTGTAGGG \\
\hline Gapdh & CAAGCTCATTTCCTGGTATGAC & СTCCTGTTATTATGGGGGTCTG \\
\hline Pou5f1 & CACTCACATCGCCAATCAGC & GGGCAGAGGAAAGGATACAG \\
\hline Gfp & AGCTGACCCTGAAGTTCATCTG & GGACTTGAAGAAGTCGTGCTG \\
\hline 18SrRNA & GTAACCCGTTGAACCCCATT & CCATCCAATCGGTAGTAGCG \\
\hline $\mathrm{Hb} 9$ & GAACACCAGTTCAAGCTCAACA & СTCTTCCGTCTTCTCCTCACTG \\
\hline Lhx3 & CGTAGCCTCTAAATGCGAGA & TGGCAAAGGTGTCTGTTCAC \\
\hline Isl1 & GTTGGAGAAAGTGGGAAATGAC & TAGAACAGACTTCATGCGCTTC \\
\hline
\end{tabular}

* Primer designs of HoxA and HoxC clusters are from the previous study (16).

ChIP-qPCR Primers
\begin{tabular}{|l|l|l|}
\hline Primer Name & Forward Sequence & Reverse Sequence \\
\hline Ch.Dm.Ubx.TSS (spike-in) & CTGAGACGGGCTAAAAGTCG & AGCACAGAAAGCGAGGAAAC \\
\hline Maz.Hoxd48.chip & TTTCTAGGGCTGGTGGTGTC & CACCCACCCTTGTCTGATG \\
\hline Maz.chipqper.cntrl & CAAAGGTCCCCATACCCAC & CTAGTCGGCCATCACTGCAA \\
\hline
\end{tabular}

\begin{tabular}{|c|c|c|c|}
\hline \multicolumn{4}{|l|}{ Antibodies } \\
\hline Antibody & Company & Catalogue \# & Application \\
\hline CTCF & Millipore & $07-729$ & ChIP, Western Blot \\
\hline MAZ & Abcam & ab85725 & ChIP, Western Blot \\
\hline $\mathrm{H} 3 \mathrm{~K} 27 \mathrm{me} 3$ & Cell Signalling & 9733 & ChIP \\
\hline $\mathrm{H} 3 \mathrm{~K} 4 \mathrm{me} 3$ & Abcam & $\mathrm{ab} 8580$ & ChIP \\
\hline RAD21 & Abcam & $\mathrm{ab} 992$ & ChIP \\
\hline RAD21 & Abcam & ab154769 & Western Blot \\
\hline CAS9 & Millipore & MAC133-clone 7A9 & Western Blot \\
\hline HB9 & Developmental Hybridoma Bank & $\mathrm{N} / \mathrm{A}$ & Western Blot \\
\hline GAPDH & Cell Signalling & D16H11-5174 & Western Blot \\
\hline B-TUBULIN & Abcam & ab6046 & Western Blot \\
\hline FLAG & Sigma & F3165 & Western Blot \\
\hline ISL1/2 & Developmental Hybridoma Bank & $\mathrm{N} / \mathrm{A}$ & Western Blot \\
\hline LHX3 & Developmental Hybridoma Bank & $\mathrm{N} / \mathrm{A}$ & Western Blot \\
\hline Histone $\mathrm{H} 3$ & Abcam & ab1791 & Western Blot \\
\hline SMC1 & Bethyl Laboratories & $\mathrm{A} 300-055 \mathrm{~A}$ & Western Blot \\
\hline $\mathrm{H} 2 \mathrm{Av}$ & Active Motif & 39715 & ChIP \\
\hline STAG1/SA1 & Abcam & $\mathrm{ab} 4457$ & Western Blot \\
\hline VINCULIN & Cell Signaling & 13901 & Western Blot \\
\hline
\end{tabular}

Table S2. List of oligos and antibodies 
bioRxiv preprint doi: https://doi.org/10.1101/2020.08.25.267237; this version posted August 26, 2020. The copyright holder for this preprint (which was not certified by peer review) is the author/funder. All rights reserved. No reuse allowed without permission.

\section{TABLE S3}

CRISPR LIBRARY PREPARATION PRIMERS, PLASMIDS

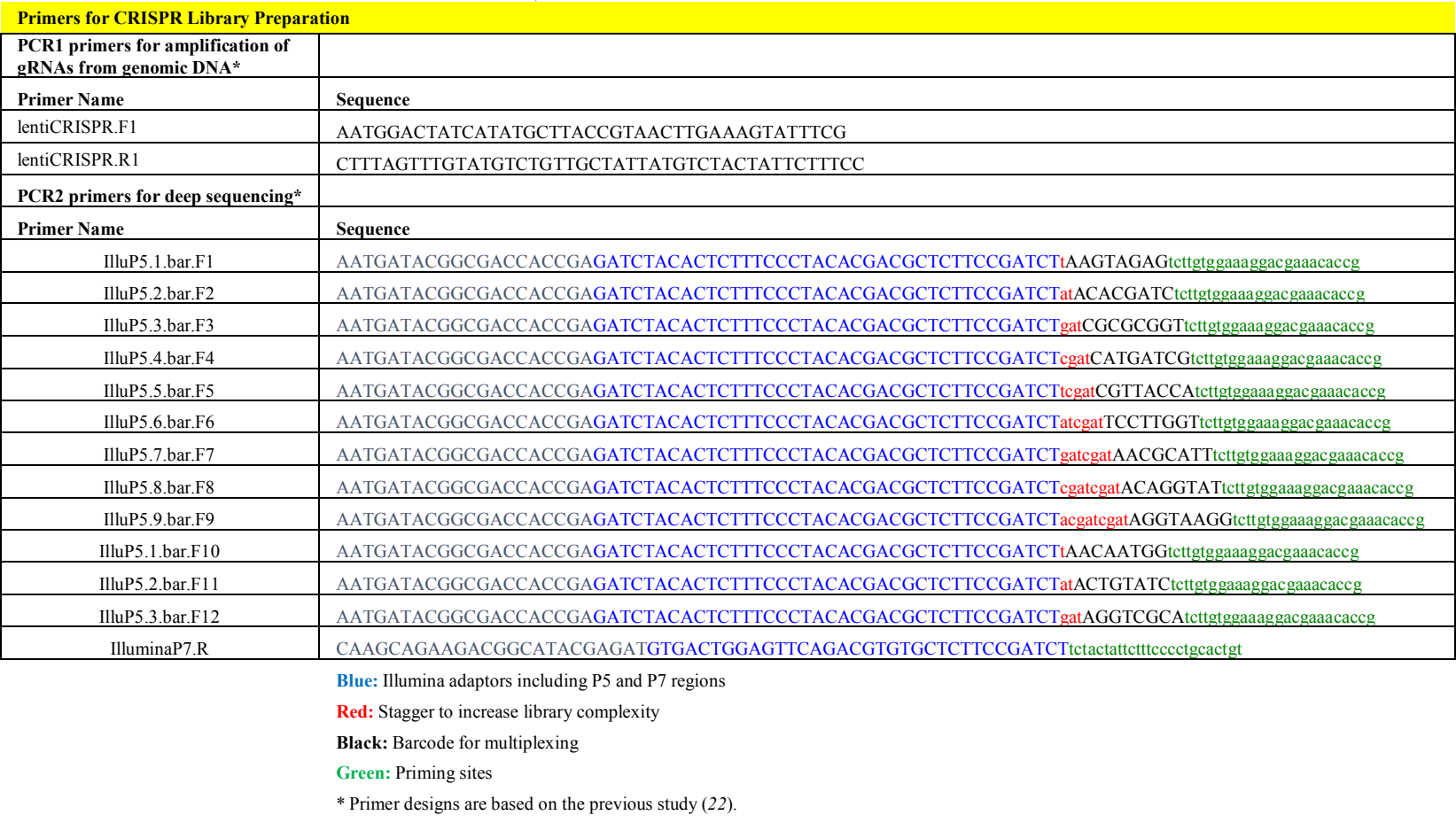

Plasmids/CRISPR Libraries
\begin{tabular}{|l|l|}
\hline Plasmid/Library Name & Addgene Numbers \\
\hline lentiCas9-blast & Addgene: 52962 \\
\hline lentiGuide-puro & Addgene: 52963 \\
\hline pSpCas9(BB)-2A-GFP (PX458) & Addgene: 48138 \\
\hline GeCKO mouse library v2 (A, B) & Addgene: 1000000053 \\
\hline CbetaS-pA (C $\beta$ F) & Addgene: 32104 \\
\hline
\end{tabular}

Table S3. List of CRISPR library preparation primers and plasmids 


\section{Data S1. (separate file)}

Common candidates identified to influence CTCF boundary in independent sub-library screens

\section{Data S2. (separate file)}

Peptide counts in native FLAG-CTCF ChIP-MS in ESCs and MNs

\section{Data S3. (separate file)}

List of sgRNAs in the custom library

\section{Data S4. (separate file)}

List of genes identified in secondary screens in WT background

\section{Data S5. (separate file)}

List of genes identified in secondary screens in CTCF site deletion background

\section{Data S6. (separate file)}

List of genes uniquely identified in secondary screens in WT background compared to CTCF site deletion background

\section{Data S7. (separate file)}

RNAseq expression values in WT vs Maz KO ESCs for differentially expressed genes

\section{Data S8. (separate file)}

RNAseq expression values in WT vs Maz KO MNs for differentially expressed genes

\section{Data S9. (separate file)}

RNAseq expression values in WT vs Maz ( $\Delta 5 \mid 6)$ ESCs for differentially expressed genes 
bioRxiv preprint doi: https://doi.org/10.1101/2020.08.25.267237; this version posted August 26, 2020. The copyright holder for this preprint (which was not certified by peer review) is the author/funder. All rights reserved. No reuse allowed without permission.

\section{Data S10. (separate file)}

RNAseq expression values in WT vs Maz $(\Delta 5 \mid 6)$ MNs for differentially expressed genes 


\section{References and Notes}

1. T. Misteli, Beyond the sequence: cellular organization of genome function. Cell 128, 787800 (2007).

2. K. Van Bortle et al., Drosophila CTCF tandemly aligns with other insulator proteins at the borders of H3K27me3 domains. Genome Res 22, 2176-2187 (2012).

3. J. E. Phillips-Cremins, V. G. Corces, Chromatin insulators: linking genome organization to cellular function. Mol Cell 50, 461-474 (2013).

4. C. T. Ong, V. G. Corces, CTCF: an architectural protein bridging genome topology and function. Nat Rev Genet 15, 234-246 (2014).

5. V. V. Lobanenkov, A novel sequence-specific DNA binding protein which interacts with three regularly spaced direct repeats of the CCCTC-motif in the 5'-flanking sequence of the chicken c-myc gene. (1990).

6. R. Ohlsson, R. Renkawitz, V. Lobanenkov, CTCF is a uniquely versatile transcription regulator linked to epigenetics and disease. Trends in Genetics 17, 520-527 (2001).

7. H. Heath et al., CTCF regulates cell cycle progression of alphabeta T cells in the thymus. EMBO J 27, 2839-2850 (2008).

8. E. Splinter et al., CTCF mediates long-range chromatin looping and local histone modification in the beta-globin locus. Genes Dev 20, 2349-2354 (2006).

9. J. R. Dixon et al., Topological domains in mammalian genomes identified by analysis of chromatin interactions. Nature 485, 376-380 (2012).

10. E. P. Nora et al., Spatial partitioning of the regulatory landscape of the X-inactivation centre. Nature 485, 381-385 (2012). 
11. F. Ciabrelli, G. Cavalli, Chromatin-driven behavior of topologically associating domains. J Mol Biol 427, 608-625 (2015).

12. J. Zlatanova, P. Caiafa, CTCF and its protein partners: divide and rule? J Cell Sci 122, 1275-1284 (2009).

13. E. D. Rubio et al., CTCF physically links cohesin to chromatin. Proc Natl Acad Sci U S A 105, 8309-8314 (2008).

14. K. S. Wendt et al., Cohesin mediates transcriptional insulation by CCCTC-binding factor. Nature 451, 796-801 (2008).

15. S. Cuddapah et al., Global analysis of the insulator binding protein CTCF in chromatin barrier regions reveals demarcation of active and repressive domains. Genome Res 19, 2432 (2009).

16. V. R. Narendra, P.P.; An, D.; Raviram, R.; Skok, J.A.; Mazzoni,E.O.; Reinberg, D., CTCF establishes discrete functional chromatin domains at the Hox clusters during differentiation. Science 347, 1017-1021 (2015).

17. A. Nitzsche et al., RAD21 cooperates with pluripotency transcription factors in the maintenance of embryonic stem cell identity. PLoS One 6, e19470 (2011).

18. F. A. Ran et al., Genome engineering using the CRISPR-Cas9 system. Nat Protoc 8, 22812308 (2013).

19. J. P. Liu, E. Laufer, T. M. Jessell, Assigning the positional identity of spinal motor neurons: rostrocaudal patterning of Hox-c expression by FGFs, Gdf11, and retinoids. Neuron 32, 997-1012 (2001).

20. E. O. Mazzoni et al., Saltatory remodeling of Hox chromatin in response to rostrocaudal patterning signals. Nat Neurosci 16, 1191-1198 (2013). 
21. N. E. Sanjana, O. Shalem, F. Zhang, Improved vectors and genome-wide libraries for CRISPR screening. Nat Methods 11, $783-784$ (2014).

22. O. Shalem et al., Genome-scale CRISPR-Cas9 knockout screening in human cells. Science 343, 84-87 (2014).

23. H. Wichterle, I. Lieberam, J. A. Porter, T. M. Jessell, Directed differentiation of embryonic stem cells into motor neurons. Cell 110, 385-397 (2002).

24. O. Shalem, N. E. Sanjana, F. Zhang, High-throughput functional genomics using CRISPRCas9. Nat Rev Genet 16, 299-311 (2015).

25. O. Parnas et al., A Genome-wide CRISPR Screen in Primary Immune Cells to Dissect Regulatory Networks. Cell 162, 675-686 (2015).

26. W. Li et al., MAGeCK enables robust identification of essential genes from genome-scale CRISPR/Cas9 knockout screens. Genome Biol 15, 554 (2014).

27. W. Li et al., Quality control, modeling, and visualization of CRISPR screens with MAGeCK-VISPR. Genome Biol 16, 281 (2015).

28. T. M. Yusufzai, H. Tagami, Y. Nakatani, G. Felsenfeld, CTCF tethers an insulator to subnuclear sites, suggesting shared insulator mechanisms across species. Mol Cell 13, 291298 (2004).

29. M. Soldi, T. Bonaldi, The ChroP Approach Combines ChIP and Mass Spectrometry to Dissect Locus-specific Proteomic Landscapes of Chromatin. Jove-Journal of Visualized Experiments, (2014).

30. C. I. Wang et al., Chromatin proteins captured by ChIP-mass spectrometry are linked to dosage compensation in Drosophila. Nat Struct Mol Biol 20, 202-209 (2013). 
31. M. R. Rafiee, C. Girardot, G. Sigismondo, J. Krijgsveld, Expanding the Circuitry of Pluripotency by Selective Isolation of Chromatin-Associated Proteins. Mol Cell 64, 624635 (2016).

32. S. Tu, G. LeRoy, D. Reinberg, Chromatin Starts to Come Clean. Mol Cell 64, 439-441 (2016).

33. K. Van Bortle et al., Insulator function and topological domain border strength scale with architectural protein occupancy. Genome Biol 15, R82 (2014).

34. J. G. Doench et al., Optimized sgRNA design to maximize activity and minimize off-target effects of CRISPR-Cas9. Nat Biotechnol 34, 184-191 (2016).

35. S. D. Bailey et al., ZNF143 provides sequence specificity to secure chromatin interactions at gene promoters. Nat Commun 2, 6186 (2015).

36. S. A. Bossone, C. Asselin, A. J. Patel, K. B. Marcu, Maz, a Zinc Finger Protein, Binds to C-Myc and C2 Gene-Sequences Regulating Transcriptional Initiation and Termination. Proceedings of the National Academy of Sciences of the United States of America 89, 74527456 (1992).

37. G. C. Kennedy, W. J. Rutter, Pur-1, a zinc-finger protein that binds to purine-rich sequences, transactivates an insulin promoter in heterologous cells. Proc. Natl. Acad. Sci. USA 89, 11498-11502 (1992).

38. J. Song et al., Two consecutive zinc fingers in $\mathrm{Sp} 1$ and in MAZ are essential for interactions with cis-elements. J Biol Chem 276, 30429-30434 (2001).

39. V. Narendra, M. Bulajic, J. Dekker, E. O. Mazzoni, D. Reinberg, CTCF-mediated topological boundaries during development foster appropriate gene regulation. Genes Dev 30, 2657-2662 (2016). 
40. M. Haller, J. Au, M. O'Neill, D. J. Lamb, 16p11.2 transcription factor MAZ is a dosagesensitive regulator of genitourinary development. Proc Natl Acad Sci U S A 115, E1849E1858 (2018).

41. D. M. Wellik, Hox genes and kidney development. Pediatr Nephrol 26, 1559-1565 (2011).

42. E. P. Nora et al., Targeted Degradation of CTCF Decouples Local Insulation of Chromosome Domains from Genomic Compartmentalization. Cell 169, 930-944 e922 (2017).

43. S. S. P. Rao et al., Cohesin Loss Eliminates All Loop Domains. Cell 171, 305-320 e324 (2017).

44. I. F. Davidson et al., DNA loop extrusion by human cohesin. Science 366, 1338-1345 (2019).

45. Y. Li et al., The structural basis for cohesin-CTCF-anchored loops. Nature 578, 472-476 (2020).

46. J. Joung et al., Genome-scale CRISPR-Cas9 knockout and transcriptional activation screening. Nat Protoc 12, 828-863 (2017).

47. O. Oksuz et al., Capturing the Onset of PRC2-Mediated Repressive Domain Formation. Mol Cell 70, 1149-1162 e1145 (2018).

48. J. D. Dignam, R. M. Lebovitz, R. G. Roeder, Accurate transcription initiation by RNA polymerase II in a soluble extract from isolated mammalian nuce. Nucleic Acids Research 11, 1475-1489 (1983).

49. S. Tu et al., Co-repressor CBFA2T2 regulates pluripotency and germline development. Nature 534, 387-390 (2016). 
50. G. LeRoy et al., LEDGF and HDGF2 relieve the nucleosome-induced barrier to transcription in differentiated cells. Sci Adv 5, eaay3068 (2019).

51. G. LeRoy, B. Rickards, S. J. Flint, The double bromodomain proteins Brd2 and Brd3 couple histone acetylation to transcription. Mol Cell 30, 51-60 (2008).

52. H. Mi, A. Muruganujan, D. Ebert, X. Huang, P. D. Thomas, PANTHER version 14: more genomes, a new PANTHER GO-slim and improvements in enrichment analysis tools. Nucleic Acids Res 47, D419-D426 (2019).

53. B. Langmead, C. Trapnell, M. Pop, S. L. Salzberg, Ultrafast and memory-efficient alignment of short DNA sequences to the human genome. Genome Biol 10, R25 (2009).

54. S. Anders, W. Huber, Differential expression analysis for sequence count data. Genome Biol 11, R106 (2010).

55. J. T. Robinson et al., Integrative genomics viewer. Nat Biotechnol 29, 24-26 (2011).

56. F. Ramirez, F. Dundar, S. Diehl, B. A. Gruning, T. Manke, deepTools: a flexible platform for exploring deep-sequencing data. Nucleic Acids Res 42, W187-191 (2014).

57. L. J. Zhu et al., ChIPpeakAnno: a Bioconductor package to annotate ChIP-seq and ChIPchip data. BMC Bioinformatics 11, 237 (2010).

58. N. T. T. Nguyen et al., RSAT 2018: regulatory sequence analysis tools 20th anniversary. Nucleic Acids Res 46, W209-W214 (2018).

59. T. L. Bailey, C. Elkan, Fitting a mixture model by expectation maximization to discover motifs in biopolymers. Proc Int Conf Intell Syst Mol Biol 2, 28-36 (1994).

60. C. Lazaris, S. Kelly, P. Ntziachristos, I. Aifantis, A. Tsirigos, HiC-bench: comprehensive and reproducible $\mathrm{Hi}-\mathrm{C}$ data analysis designed for parameter exploration and benchmarking. BMC Genomics 18, 22 (2017). 
61. B. Langmead, S. L. Salzberg, Fast gapped-read alignment with Bowtie 2. Nat Methods 9, 357-359 (2012).

62. M. Imakaev et al., Iterative correction of Hi-C data reveals hallmarks of chromosome organization. Nat Methods 9, 999-1003 (2012).

63. S. Heinz et al., Simple combinations of lineage-determining transcription factors prime cis-regulatory elements required for macrophage and B cell identities. Mol Cell 38, 576589 (2010).

64. S. Heinz et al., Transcription Elongation Can Affect Genome 3D Structure. Cell 174, 15221536 e1522 (2018).

65. A. Kloetgen et al., Three-dimensional chromatin landscapes in T cell acute lymphoblastic leukemia. Nat Genet 52, 388-400 (2020).

66. F. Ay, T. L. Bailey, W. S. Noble, Statistical confidence estimation for Hi-C data reveals

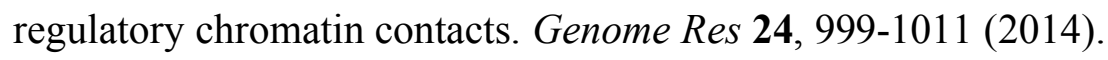

67. S. S. Rao et al., A 3D map of the human genome at kilobase resolution reveals principles of chromatin looping. Cell 159, 1665-1680 (2014).

68. M. T. Weirauch et al., Determination and inference of eukaryotic transcription factor sequence specificity. Cell 158, 1431-1443 (2014).

69. G. Ambrosini, R. Groux, P. Bucher, PWMScan: a fast tool for scanning entire genomes with a position-specific weight matrix. Bioinformatics 34, 2483-2484 (2018).

70. F. Ramirez et al., High-resolution TADs reveal DNA sequences underlying genome organization in flies. Nat Commun 9, 189 (2018). 